\title{
Coronal Loops: \\ Observations and Modeling of Confined Plasma
}

\author{
Fabio Reale \\ Dipartimento di Fisica \& Chimica, \\ Università di Palermo, \\ Piazza Parlamento 1, 90134 Palermo, Italy \\ email: reale@astropa.unipa.it \\ http://www.astropa.unipa.it/ reale/
}

Accepted: 23 June 2014

Published: 29 July 2014

(Update of lrsp-2010-5)

\begin{abstract}
Coronal loops are the building blocks of the X-ray bright solar corona. They owe their brightness to the dense confined plasma, and this review focuses on loops mostly as structures confining plasma. After a brief historical overview, the review is divided into two separate but not independent parts: the first illustrates the observational framework, the second reviews the theoretical knowledge. Quiescent loops and their confined plasma are considered and, therefore, topics such as loop oscillations and flaring loops (except for non-solar ones, which provide information on stellar loops) are not specifically addressed here. The observational section discusses the classification, populations, and the morphology of coronal loops, its relationship with the magnetic field, and the loop stranded structure. The section continues with the thermal properties and diagnostics of the loop plasma, according to the classification into hot, warm, and cool loops. Then, temporal analyses of loops and the observations of plasma dynamics, hot and cool flows, and waves are illustrated. In the modeling section, some basics of loop physics are provided, supplying fundamental scaling laws and timescales, a useful tool for consultation. The concept of loop modeling is introduced and models are divided into those treating loops as monolithic and static, and those resolving loops into thin and dynamic strands. More specific discussions address modeling the loop fine structure and the plasma flowing along the loops. Special attention is devoted to the question of loop heating, with separate discussion of wave (AC) and impulsive (DC) heating. Large-scale models including atmosphere boxes and the magnetic field are also discussed. Finally, a brief discussion about stellar coronal loops is followed by highlights and open questions.
\end{abstract}

Keywords: Coronal loops, Corona

This review is licensed under a Creative Commons Attribution-Non-Commercial 3.0 Germany License. http://creativecommons . org/licenses/by-nc/3.0/de/ 


\section{Imprint / Terms of Use}

Living Reviews in Solar Physics is a peer reviewed open access journal published by the Max Planck Institute for Solar System Research, Justus-von-Liebig-Weg 3, 37077 Göttingen, Germany. ISSN 1614-4961.

This review is licensed under a Creative Commons Attribution-Non-Commercial 3.0 Germany License: http://creativecommons.org/licenses/by-nc/3.0/de/. Figures that have been previously published elsewhere may not be reproduced without consent of the original copyright holders.

Because a Living Reviews article can evolve over time, we recommend to cite the article as follows:

Fabio Reale,

"Coronal Loops: Observations and Modeling of Confined Plasma", Living Rev. Solar Phys., 11, (2014), 4. URL (accessed < date>): http://www.livingreviews.org/lrsp-2014-4

The date given as $<$ date $>$ then uniquely identifies the version of the article you are referring to. 


\section{Article Revisions}

Living Reviews supports two ways of keeping its articles up-to-date:

Fast-track revision. A fast-track revision provides the author with the opportunity to add short notices of current research results, trends and developments, or important publications to the article. A fast-track revision is refereed by the responsible subject editor. If an article has undergone a fast-track revision, a summary of changes will be listed here.

Major update. A major update will include substantial changes and additions and is subject to full external refereeing. It is published with a new publication number.

For detailed documentation of an article's evolution, please refer to the history document of the article's online version at http: //www. livingreviews .org/lrsp-2014-4.

29 July 2014: Substantially revised and updated the previous version. Added 4 new figures. The number of references increased from 396 to 557 . 


\section{Contents}

1 Introduction $\quad 5$

2 Historical Keynotes $\quad 7$

3 The Observational Framework $\quad 9$

3.1 General properties . . . . . . . . . . . . . . . . . . . . . 9

3.1 .1 Classification . . . . . . . . . . . . . . . . . . . 11

3.2 Morphology and fine structuring . . . . . . . . . . . . . . . . . . . 12

3.2 .1 Geometry . . . . . . . . . . . . . . . . . . . . . 12

3.2 .2 Fine structuring . . . . . . . . . . . . . . . . . . . 15

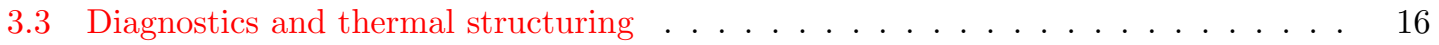

3.3 .1 Hot loops . . . . . . . . . . . . . . . . . . . . . . . . . . . 20

3.3.2 Comparison of hot and warm loops . . . . . . . . . . . . . . . 21

3.3 .3 Warm loops . . . . . . . . . . . . . . . . . . . . . . . . 24

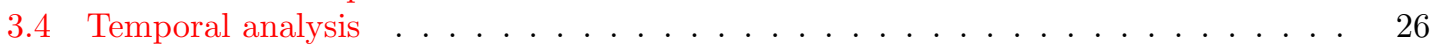

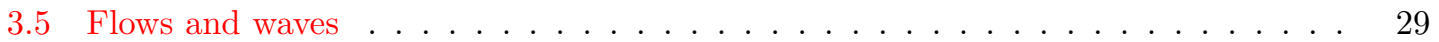

3.5 .1 Chromospheric flows . . . . . . . . . . . . . . . . . . 32

3.5.2 Waves observations . . . . . . . . . . . . . . . . . . . . . 32

4 Loop Physics and Modeling $\quad \mathbf{3 4}$

4.1 Basics . . . . . . . . . . . . . . . . . . . . . . 34

4.1.1 Monolithic (static) loops: scaling laws . . . . . . . . . . . . . . 37

4.1 .2 Structured $($ dynamic) loops . . . . . . . . . . . . . . . . . . . 38

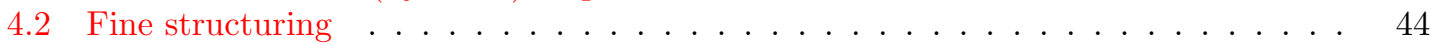

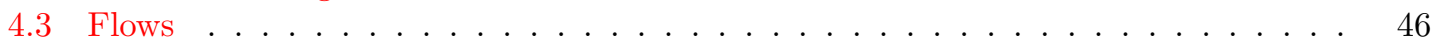

4.4 Heating . . . . . . . . . . . . . . . . . . . . . . 49

4.4 .1 DC heating . . . . . . . . . . . . . . . . . . . . 51

4.4 AC heating . . . . . . . . . . . . . . . . . . . . . . 53

4.5 Large-scale modeling . . . . . . . . . . . . . . . . . . . . . . . . . . 55

$\begin{array}{lll}5 & \text { Stellar Coronal Loops } & 57\end{array}$

6 Conclusions and Perspectives $\quad 58$

$\begin{array}{ll}\text { References } & 61\end{array}$

\section{List of Tables}

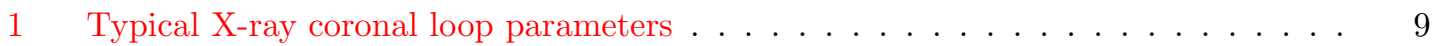

2 Thermal coronal loop classification . . . . . . . . . . . . . . . . . . . 11 


\section{Introduction}

The corona is the outer part of the solar atmosphere. Its name derives from the fact that, since it is extremely tenuous with respect to the lower atmosphere, it is visible in the optical band only during the solar eclipses as a faint crown (corona in Latin) around the black moon disk. When inspected through spectroscopy the corona reveals unexpected emission lines, which were first identified as due to a new element (coronium), but which were later ascertained to be due to high excitation states of iron (Grotrian, 1939; Edlén, 1943). It became then clear that the corona is made of very high temperature gas, hotter than $1 \mathrm{MK}$. Almost all the gas is fully ionized there and thus interacts effectively with the ambient magnetic field. It is for this reason that the corona appears so inhomogeneous when observed in the X-ray band, in which plasma at million degrees emits most of its radiation. In particular, the plasma is confined inside magnetic flux tubes that are anchored on both sides to the underlying photosphere. When the confined plasma is heated more than the surroundings, its pressure and density increase. Since the tenuous plasma is optically thin, the intensity of its radiation is proportional to the square of the density, and the tube becomes much brighter than the surrounding ones and looks like a bright closed arch: a coronal loop.

When observed in the X-ray band, the bright corona appears to be made entirely by coronal loops that can, therefore, be considered as the building blocks of X-ray bright corona. This review specifically addresses coronal loops as bright structures confining plasma. It first provides an observational framework that is the basis for the second part of the review dealing with modeling and interpretation.

The observational section (3) discusses loop classification and populations, and then describes the morphology of coronal loops, its relationship with the magnetic field, regarding the shape and cross-section, and the concept of loops as consisting of bundles of strands, whose thickness may go down to sub-arcsecond scale. The following part of this section is devoted to the characteristics of the loop plasma and of its thermal structure. Diagnostics of the emission measure and of its temperature distribution retrieved from filter ratios and spectroscopy are introduced. The thermal properties of the loops are discussed according to a broad classification into hot, warm, and cool loops. Hot loops are best observed in the soft X-rays and in active regions, and attention is devoted to the possible presence of minor very hot components out of flares. Warm loops are those better observed in several EUV bands with lines emitted around $1 \mathrm{MK}$ and often found to be more isothermal and dense than expected, probably because they are out of equilibrium. The emission measure distribution of loops in the whole coronal temperature range is reviewed paying attention to its shape and broadness, that may indicate or not the coexistence of many heatingcooling cycles. Then, temporal analyses of loop light curves focus on searching for variability in different bands that may indicate a highly variable or more steady heating, and the characteristic timescales. The observations show more and more evidence for significant plasma dynamics and flows, from subsonic to supersonic. Widespread patterns of redshifts and blueshifts are found in different temperature regimes and their spatial distribution is also discussed. Evidence for upflows from the chromosphere and of possible coronal counterparts is addressed. Observations reporting on the detection of waves propagating along loops are also mentioned.

In the modeling section (4) some basics of loop physics are provided, supplying some fundamental scaling laws and timescales, a useful tool for consultation. The concept of loop modeling is introduced and models are distinguished between those treating loops as monolithic and static, and those resolving loops into thin and dynamic strands. Then, more specific discussions address how modeling the loop fine structure is able to explain observed evidence for deviations from equilibrium and different filling factors in different bands, and can help investigating the concept of randomly-distributed heat pulses. Models also address plasma flowing along the loops, both as siphon flows and as motions driven by dynamic heating, i.e., hot upflows from the chromosphere first and downflows from draining afterwards. Special attention is devoted to the question of loop 
heating, which is strictly connected to the general problem of coronal heating. The conversion of magnetic energy into heat and the problem of the difficult diagnostics of the heating are first discussed in general terms. Impulsive (DC) and wave (AC) heating are separately discussed. DC models have extensively investigated the heating by nanoflares searching for possible signatures and properties such as their frequency and location. AC heating models focus on the way to dissipate waves and to match the observational scenario. Some discussion is devoted to MHD models that describe the solar atmosphere from the chromosphere to the corona on a larger area, and including the magnetic field and the radiative transfer, and to models that describe the magnetic field dissipation through turbulent cascades to very small scales.

There have been several earlier books (Bray et al., 1991; Golub and Pasachoff, 1997, 2001; Aschwanden, 2004) and reviews (Vaiana and Rosner, 1978; Peres and Vaiana, 1990; Golub, 1996; Aschwanden et al., 2001; Reale, 2005), in particular on coronal heating (Zirker, 1993; Cargill, 1995; Klimchuk, 2006; De Moortel and Nakariakov, 2012; Parnell and De Moortel, 2012), that have in general a larger or different scope but include information about coronal loops. Interested readers are urged to survey these other reviews in order to complement and fill in any gaps in topical coverage of the present paper. 


\section{Historical Keynotes}

First evidence of magnetic confinement came from rocket missions in the 1960s. In particular, in 1965, arcmin angular resolution was achieved with grazing incidence optics (Giacconi et al., 1965). The data analysis led to the first density and temperature diagnostics with wide band filters, to derive high pressure in compact regions with intense bipolar magnetic fields and to propose the magnetic confinement (Reidy et al., 1968). The first coronal loop structures were identified properly after a rocket launch in 1968, which provided for the first time an image of an X-ray flare (Vaiana et al., 1968), with a resolution of a few arcsec.

In the course of collecting the results of all rocket missions of the American Science and Engineering (AS\&E) program, Vaiana et al. (1973) proposed a classification of the morphology of the X-ray corona as fundamentally consisting of arch-like structures connecting regions of opposite magnetic polarity in the photosphere. The classification was based on the loop size, and on the physical conditions of the confined plasma, on the underlying photospheric regions. They distinguished active regions, coronal holes, active regions interconnection, filament cavities, bright points, and large-scale structures (Vaiana and Rosner, 1978; Peres and Vaiana, 1990).

The magnetic structuring of the solar corona is evident. However, the magnetic field lines can be traced only indirectly because direct measurements are feasible generally only low in the photosphere through the Zeeman effect on spectral lines. It is anyhow possible to extrapolate the magnetic field in a volume. This was done to derive the magnetic field structure of a relatively stable active region by Poletto et al. (1975) using the Schmidt (1964) method, under the assumption of negligible currents in the corona. This was also useful to derive magnetic field intensities sufficient for hot plasma confinement. Later on, even more reliable magnetic field topologies were derived assuming force-free fields (e.g., Sakurai, 1981), i.e., with currents everywhere parallel to the magnetic field as it is expected in coronal loops. However, the agreement of force-free magnetic field extrapolation with the details of the observed coronal EUV topology is often far from satisfactory (e.g., Wiegelmann et al., 2006).

The rocket missions lacked good time coverage and the information about the evolution of coronal loops was only limited, mostly available from the Orbiting Solar Observatory-IV (OSOIV) mission (Krieger et al., 1972). This satellite had an angular resolution in the order of the arcmin and could not resolve individual loops. In 1973, the X-ray telescope S-054 on-board Skylab monitored the evolution of coronal loops for several months, taking $32000 \mathrm{X}$-ray photographs with a maximum resolution of 2 arcsec and an extended dynamic range. It was possible to study the whole evolution of an active region, from the emergence as compact loops filled with dense plasma to its late spreading, a few solar rotations later, as progressively longer and longer loops filled with less and less dense plasma (Golub et al., 1982). It was confirmed that the whole X-ray bright corona consists of magnetic loops, whose lifetime is typically much longer than the characteristic cooling times (Rosner et al., 1978). This applies also to coronal holes where the magnetic field opens radially to the interplanetary space and the plasma streams outwards with practically no X-ray emission.

In the same mission coronal loops were also detected in the UV band at temperatures below $1 \mathrm{MK}$, by Extreme UltraViolet (EUV) telescopes S-055 (Reeves et al., 1977) and S-082 (Tousey et al., 1977; Bartoe et al., 1977). These loops are invisible in the X-ray band and many of them depart from sunspots, appear coaxial and are progressively thinner for progressively lower temperature ions (Foukal, 1975, 1976). The apparent scale height of the emission is larger than that expected from a static model, but the loops appear to be steady for long times. Foukal (1976) proposed a few explanations including siphon flows and thermal instability of the plasma at the loop apex. New observations of such cool loops were performed several years later with the Solar and Heliospheric Observatory (SoHO) mission and provided new details and confirmations (Section 3.5). 
A different target was addressed by the Solar Maximum Mission (SMM, 1980-1989, Bohlin et al., 1980; Acton et al., 1980), which included high-resolution spectrometers in several X-ray lines, i.e., the Bent Crystal Spectrometer (BCS) and the Flat Crystal Spectrometer (FCS), mostly devoted to obtain time-resolved spectroscopy of coronal flares (e.g., MacNeice et al., 1985). Similarly, the Hinotori mission (1981-1991, Tanaka, 1983) was dedicated mainly to solar flare observations in the X-ray band. This was also the scope of the later Yohkoh mission, (1991-2001, Ogawara et al., 1991) by means of high resolution X-ray spectroscopy, adding the monitoring and imaging of the hot and flaring corona. Hara et al. (1992) found first indications of plasma at 5-6 MK in active regions with the Soft X-ray Telescope (SXT, Tsuneta et al., 1991).

Normal-incidence optics were developed in the late 1980s. An early experiment was the Normal Incidence X-ray Telescope (NIXT, Golub and Herant, 1989), which provided a few high resolution coronal images in the EUV band.

Later space missions dedicated to study the corona have been the Solar and Heliospheric Observatory (SoHO, Domingo et al., 1995), launched in 1995 and still operative, and the Transition Region and Coronal Explorer (TRACE, Handy et al., 1999), launched in 1998 and replaced in 2010 by the Solar Dynamic Observatory (SDO) instruments. Both SoHO and TRACE were tailored to observe the quiet corona (below $2 \mathrm{MK}$ ). SoHO images the whole corona (Extreme ultraviolet Imaging Telescope, EIT, Delaboudinière et al., 1995) and performs wide band spectroscopy (Solar Ultraviolet Measurements of Emitted Radiation, SUMER, Wilhelm et al., 1995) and (Coronal Diagnostic Spectrometer, CDS, Harrison et al., 1995) in the EUV band; TRACE imaged the EUV corona with high spatial $(0.5$ arcsec) and temporal (30 s) resolution. Both SoHO/EIT and TRACE are based on normal-incidence optics and contain three different EUV filters that provide limited thermal diagnostics.

Thanks to their capabilities, both missions allowed to address finer diagnostics, in particular to investigate the fine transverse structuring of coronal loops, both in its geometric and thermal components, and the plasma dynamics and the heating mechanisms at a higher level of detail. SoHO and TRACE have been complementary in many respects and several studies attempted to couple the information from them.

Among other relevant missions, we mention the CORONAS series (Ignatiev et al., 1998; Oraevsky and Sobelman, 2002), with instruments like SPectroheliographIc X-Ray Imaging Telescope (SPIRIT, Zhitnik et al., 2003), REntgenovsky Spektrometr s Izognutymi Kristalami (ReSIK, Sylwester et al., 1998), and Solar Photometer in X-rays (SPHINX, Sylwester et al., 2008; Gburek et al., 2013), which have contributed to the investigation of coronal loops.

In late 2006, two other major solar missions started, namely Hinode (Kosugi et al., 2007) and the Solar TErrestrial Relations Observatory (STEREO, e.g., Kaiser et al., 2008). On-board Hinode, two instruments address particularly the study of coronal loops: the X-Ray Telescope (XRT, Golub et al., 2007) and the Extreme-ultraviolet Imaging Spectrometer (EIS, Culhane et al., 2007). Both these instruments offer considerable improvements on previous missions. The XRT has a spatial resolution of about 1 arcsec, a very low scattering and the possibility to switch among nine filters and combinations of them. EIS combines well spectral $(\sim 2 \mathrm{~mA})$, spatial $\left(2^{\prime \prime}\right)$, and temporal $(\sim 10 \mathrm{~s})$ resolution to obtain accurate diagnostics of plasma dynamics and density. One big achievement of the STEREO mission is that, since it consists of two separate spacecrafts getting farther and farther from each other, it allows - through, for instance, its Sun-Earth Connection Coronal and Heliospheric Investigation (SECCHI) package - a first 3D reconstruction of coronal loops (Aschwanden et al., 2009; Kramar et al., 2009).

In 2010, the Solar Dynamics Observatory (SDO, Pesnell et al., 2012) mission has been launched with three instruments on-board: Atmospheric Imaging Assembly (AIA, Lemen et al., 2012; Boerner et al., 2012), EUV Variability Experiment (EVE, Woods et al., 2012), and Helioseismic and Magnetic Imager (HMI, Scherrer et al., 2012). SDO observations lead to big improvements in the study of coronal-loop physics, basically because it monitors the full Sun continuously with 
high temporal and spatial resolution, especially with the AIA EUV normal-incidence telescope at 9 different UV and EUV channels. It is worthwhile mentioning also the sounding rocket mission High-resolution Coronal Imager (Hi-C, Cirtain et al., 2013), which achieved an unprecedented spatial resolution $\left(0.2^{\prime \prime}\right)$ in the EUV band $(195 \AA)$.

\section{The Observational Framework}

\subsection{General properties}

Although coronal loops are often well defined and studied in the EUV band, detected by many space mission spectrometers like those on board SoHO and Hinode, and by high resolution imagers such as TRACE and SDO/AIA, the bulk of coronal loops is visible in the X-ray band (Figure 1). Also, the peak of the coronal emission measure of active regions - where the loops are brightest - is above 2 MK, which is best observed in X-rays (e.g., Peres et al., 2000; Reale et al., 2009a; Warren et al., 2011).

Coronal loops are characterized by an arch-like shape that recalls typical magnetic field topology. This shape is replicated over a wide range of dimensions. Referring, for the moment, to the soft X-ray band, the main properties of coronal loops are listed in Table 1. The length of coronal loops spans more than two orders of magnitude. As already mentioned, the loops owe their high luminosity and variety to their nature of magnetic flux tubes where the plasma is confined and isolated from the surroundings. Magnetized fully-ionized plasma conducts thermal energy mostly along the magnetic field lines. Due to the high thermal insulation, coronal loops can have different temperatures, from $\sim 10^{5} \mathrm{~K}$ up to a few $\sim 10^{7} \mathrm{~K}$ (flaring loops). A density of the confined plasma below $10^{7}-10^{8} \mathrm{~cm}^{-3}$ can be difficult to detect, while the density can grow up to $10^{12} \mathrm{~cm}^{-3}$ in flaring loops. The corresponding plasma pressure in non-flaring loops can typically vary between $10^{-3}$ and 10 dyne $\mathrm{cm}^{-2}$, corresponding to confining magnetic fields $B \sim 8 \pi p^{0.5}$ of the order of $0.1-10 \mathrm{G}$ in the corona. One characterizing feature of coronal loops is that typically their crosssection is constant along their length above the transition region, at variance from the topology of potential magnetic fields. There is evidence that the cross-section varies across the transition region, as documented in Gabriel (1976). A simple geometric description is reported in Chae et al. (1998c):

$$
A(T) / A\left(T_{h}\right)=\left[1+\left(\Lambda^{2}-1\right)\left(T / T_{h}\right)^{\nu}\right]^{1 / 2} / \Lambda,
$$

where $A$ is the cross-section area, $T$ is the temperature, $T_{h}=10^{6} \mathrm{~K}, \Lambda=30$, and $\nu=3.6$.

Table 1: Typical X-ray coronal loop parameters

\begin{tabular}{lcccc}
\hline Type & $\begin{array}{c}\text { Length } \\
{\left[10^{9} \mathrm{~cm}\right]}\end{array}$ & $\begin{array}{c}\text { Temperature } \\
{[\mathrm{MK}]}\end{array}$ & $\begin{array}{c}\text { Density } \\
{\left[10^{9} \mathrm{~cm}^{-3}\right]}\end{array}$ & $\begin{array}{c}\text { Pressure } \\
{\left[\text { dyne } \mathrm{cm}^{-2}\right]}\end{array}$ \\
\hline Bright points & $0.1-1$ & 2 & 5 & 3 \\
Active region & $1-10$ & 3 & $1-10$ & $1-10$ \\
Giant arches & $10-100$ & $1-2$ & $0.1-1$ & 0.1 \\
Flaring loops & $1-10$ & $>10$ & $>50$ & $>100$ \\
\hline
\end{tabular}



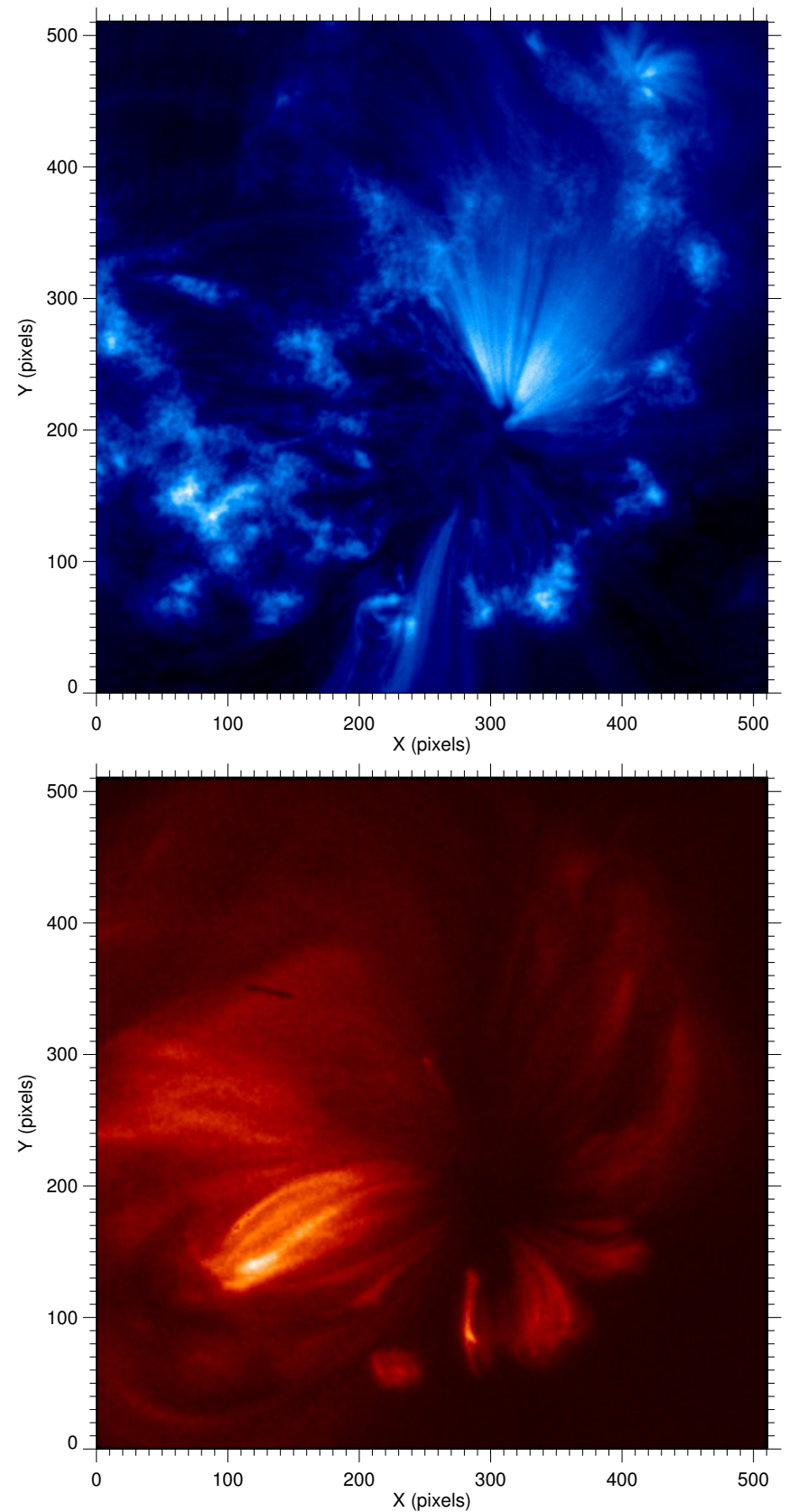

Figure 1: Images of the same active region, taken in the EUV band with TRACE (top) and in the X-ray band with Hinode/XRT (bottom), on 14 November 2006. The X-ray image shows more clearly that the active region is densely populated with coronal loops. 


\subsubsection{Classification}

Myriads of loops populate the solar corona and constitute statistical ensembles. Attempts to define and classify coronal loops were never easy, and no finally established result exists to-date. Early attempts were based on morphological criteria, i.e., bright points, active-region loops, and large-scale structures (Vaiana et al., 1973, Figure 2), largely observed with instruments in the $\mathrm{X}$-ray band. In addition to such classification, more recently, the observation of loops in different spectral bands and the suspicion that the difference lies not only in the band, but also in intrinsic properties, have stimulated another classification based on the temperature regime, i.e., cool, warm, hot loops (Table 2). Cool loops are generally detected in UV lines at temperatures between $10^{5}$ and $10^{6} \mathrm{~K}$. They were first addressed by Foukal (1976) and later explored more with SoHO observations (Brekke et al., 1997). Warm loops are well observed by EUV imagers such as SoHO/EIT, TRACE, and in most channels of SDO/AIA, and confine plasma at temperature around 1-1.5 MK (Lenz et al., 1999). Hot loops are those typically observed in the X-ray band, and in hot UV and EUV lines (e.g., FeXVI) and channels (SDO/AIA $335 \AA$ ), with temperatures around or above 2 MK (Table 1). These are the coronal loops already identified, for instance, in the early rocket missions (Vaiana et al., 1973). This distinction is not only due to observation with different instruments and in different bands, but there are hints that it may be more substantial and physical, i.e., there may be two or more classes of loops that may be governed by different regimes of physical processes. For instance, the temperature along warm loops appears to be distributed uniformly and the density to be higher than that predicted by equilibrium conditions. Does this make such loops intrinsically different from hot loops, or is it just the signature that warm loops are a transient conditions of hot loops? New state-of-art methods, like differential emission measure tomography (DEMT), have proposed a new classification of coronal loops based on whether the temperature increases or decreases with height (Huang et al., 2012).

Table 2: Thermal coronal loop classification

\begin{tabular}{lc}
\hline Type & Temperature $[\mathrm{MK}]$ \\
\hline Cool & $0.1-1$ \\
Warm & $1-1.5$ \\
Hot & $\geq 2$ \\
\hline
\end{tabular}

A real progress in the insight into coronal loops is expected from the study of large samples of loops or of loop populations. Systematic studies of coronal loops suffer from the problem of the sample selection and loop identification, because, for instance, loops in active regions overlap along the line sight. Attempts of systematic studies have been performed in the past on Yohkoh and TRACE data (e.g., Porter and Klimchuk, 1995; Aschwanden et al., 2000). A large number of loops were analyzed and it was possible to obtain meaningful statistics. However, it is difficult to generalize the results because of limited samples and/or selection effects, e.g., best observed loops, specific instrument. One basic problem for statistical studies of coronal loops is that it is very difficult to define an objective criterion for loop identification. In fact, loops are rarely isolated; they coexist with other loops that intersect or even overlap along the line of sight. This is especially true in active regions where most of the loops are found. In order to make a real progress along this line, we should obtain loop samples and populations selected on totally objective and unbiased criteria, which is difficult due to the problems outlined above. Some steps are coming in this direction (Aschwanden et al., 2013) and we will see results in the future. 


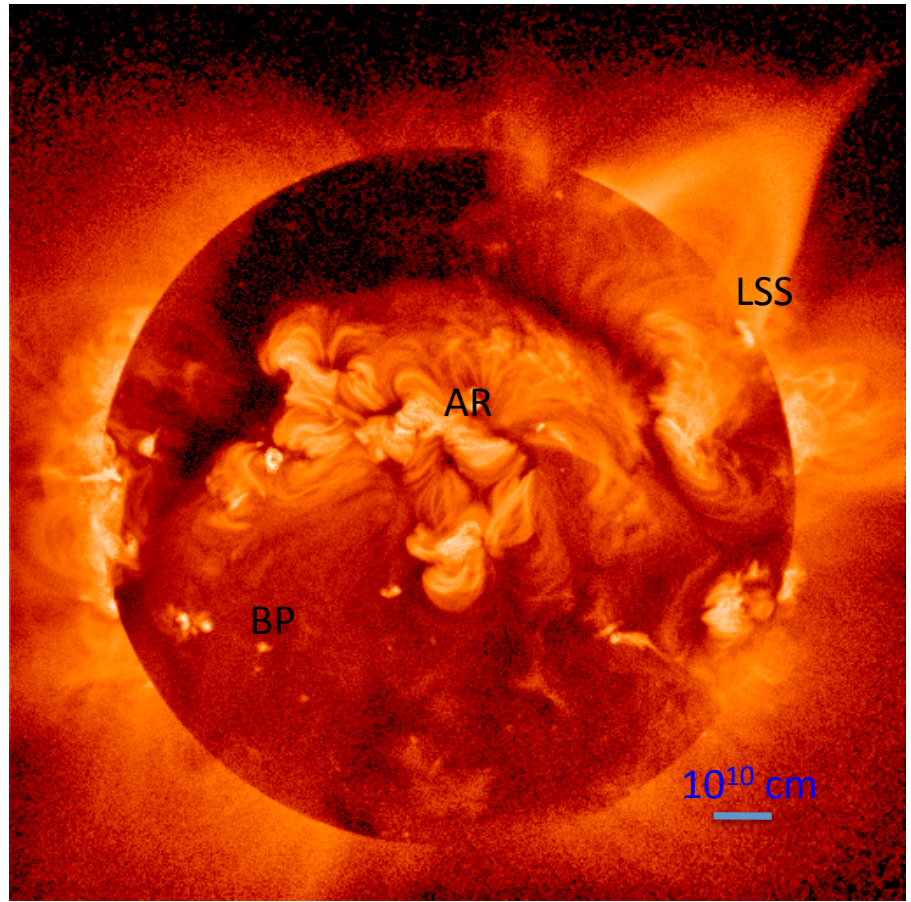

Figure 2: The X-ray corona contains loops with different spatial scales, e.g., bright points (BP), active region loops (AR), large-scale structures (LSS). The scale unit is labelled. Image credit: Yohkoh mission, ISAS, Japan.

\subsection{Morphology and fine structuring}

\subsubsection{Geometry}

Coronal loops are magnetic structures and might therefore be mapped easily and safely by mapping reliably the coronal magnetic field. Unfortunately, it is well-known that it is very difficult to measure the magnetic field in the corona, and it can be done only in very special conditions, e.g., very strong local field (White et al., 1991). In some cases it is possible to use coronal seismology (first proposed by Uchida, 1970) to determine the average magnetic field strength in an oscillating loop, first used by Nakariakov et al. (1999) and Nakariakov and Ofman (2001), on TRACE loops, and more recently investigated in a number of studies. In some of these studies, for instance, seismological techniques are used in order to measure flare-induced loop oscillations (Ballai et al., 2011) and waves and flows in active region loops (Wang et al., 2013; Uritsky et al., 2013). The accuracy of these methods depends on the correct detection of the temporally and spatially resolved mode of oscillation, and on the details of the loop geometry.

Since we cannot determine well the coronal magnetic field, coronal loop geometry deserves specific analysis. As a good approximation, loops generally have a semicircular shape (Figure 3). The loop aspect, of course, depends on the loop orientation with respect to the line of sight: loops with the footpoints on the limb more easily appear as semicircular, as well as loops very inclined on the surface near the center of the disk. The assumption of semicircular shape can be useful to measure the loop length even in the presence of important deformations due to projection effects: the de-projected distance of the loop footpoints is the diameter of the arc. However, deviations from circularity are rather common and, in general, the detailed analysis of the loop geometry is not a trivial task. The accurate determination of the loop geometry is rather important for the 


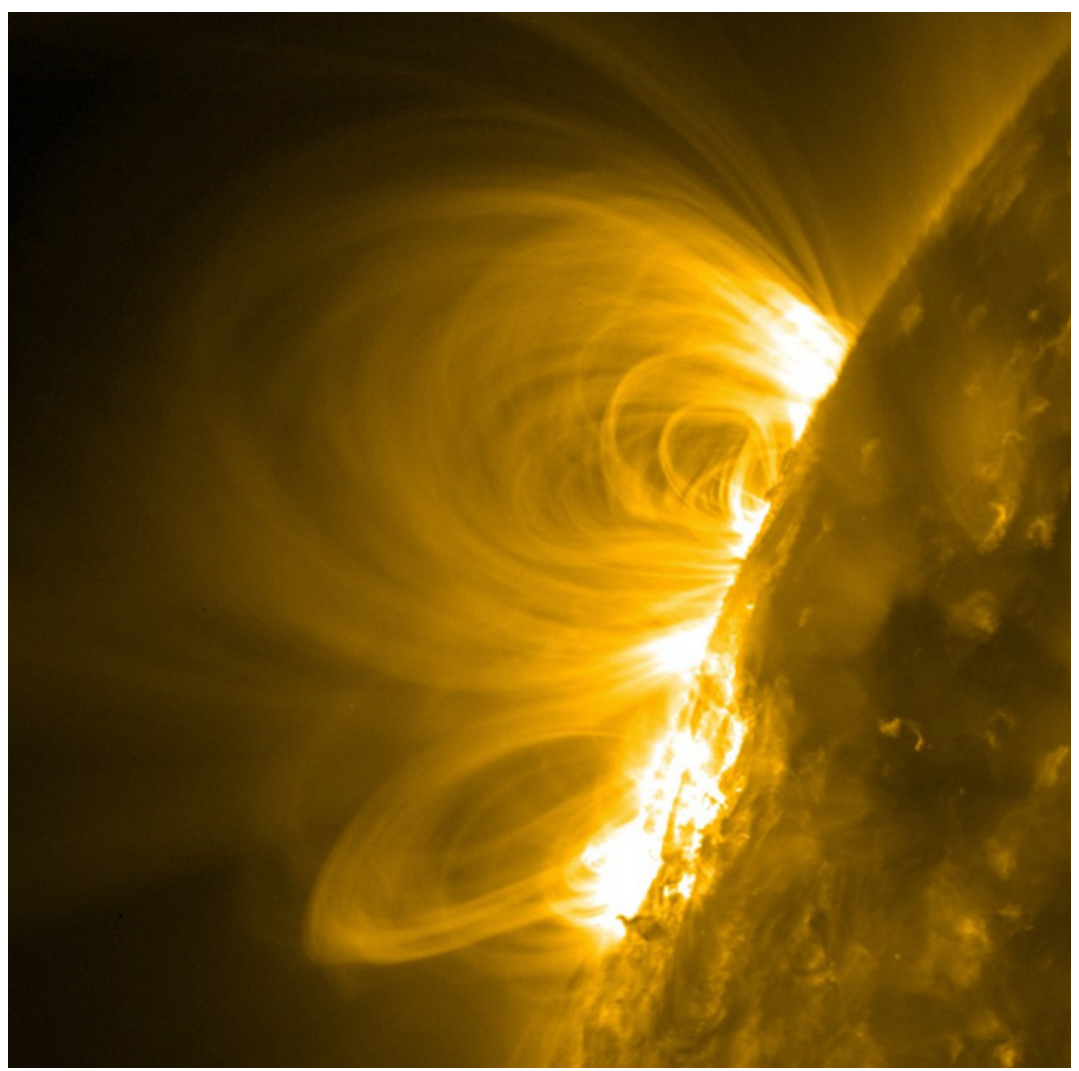

Figure 3: Coronal loops have approximately a semicircular shape. Image: SDO/AIA, $171 \AA$ filter, 24 February 2011. Credit: NASA/LMSAL/SDO.

implications on the magnetic field topology and reconstruction. It is less important for the structure and evolution of the confined plasma, which follow the field lines whatever shape they have and change little also with moderate changes of the gravity component along the field lines. First works on the accurate determination of the loop geometry date back to the 1960s (Saito and Billings, 1964). More specific ones take advantage of stereoscopic views allowed by huge loops during solar rotation, with the aid of magnetic field reconstruction methods. These studies find deviations from ideal circularity and symmetry, not surprising for such large structures (Berton and Sakurai, 1985). The geometry of a specific loop observed with TRACE was measured in the framework of a complete study including time-dependent hydrodynamic modeling (Reale et al., 2000a,b). In that case, the discrepancy between the length derived from the distance of the footpoints taken as loop diameter and the length measured along the loop itself allowed to assess the loop as elongated. Later, a reconstruction of loop geometry was applied to TRACE observation of medium-sized oscillating loops, to derive the properties of the oscillations. In this case, a semicircular pattern was applied (Aschwanden et al., 2002). The importance of the deviations from circularity on constraining loop oscillations was remarked later (Dymova and Ruderman, 2006).

The STEREO mission is contributing much to the analysis of loop morphology and geometry, thanks to its unique capability to observe the Sun simultaneously from different positions. The three-dimensional shape of magnetic loops in an active region was first stereoscopically reconstructed from two different vantage points based on simultaneously recorded STEREO/SECCHI images (Feng et al., 2007). Five relatively long loops were measured and found to be non-planar 
and more curved than field lines extrapolated from SoHO/MDI measurements, probably due to the inadequacy of the linear force-free field model used for the extrapolation. A misalignment of 2040 deg between theoretical model and observed loops has been quantified from STEREO results (Sandman et al., 2009; DeRosa et al., 2009). Systematic triangulations and 3D reconstructions using the Extreme UltraViolet Imager (EUVI) telescopes on both STEREO spacecrafts were used to derive loop characteristics, such as the loop plane inclination angles (Aschwanden et al., 2008, 2009, 2012). Deviations from circularity within 30\%, less significant from coplanarity and twisting below the threshold for kink instability, were found.

Another interesting issue regarding coronal loop geometry is the analysis of the loop crosssection, which also provides information about the structure of the coronal magnetic field. Yohkoh/SXT allowed for systematic and quantitative studies of loop morphology and showed that the cross-section of coronal loops is approximately constant along their length and do not increase significantly. More in detail, a systematic analysis of a sample of ten loops showed that the loops tend to be only slightly $(\sim 30 \%)$ wider at their midpoints than at their footpoints, while for a bipolar field configuration we would expect expansions by factors. One possible explanation of this effect is the presence of significant twisting of the magnetic field lines and, therefore, the development of electric currents and strong deviations from a potential field. The effect might be seen either as a twisting of a single loop or as a "braiding" of a bundle of unresolved thin loops (see Section 3.2.2). At the same time it was found that the variation of width along each loop tends to be modest, implying that the cross section has an approximately circular shape (Klimchuk et al., 1992).

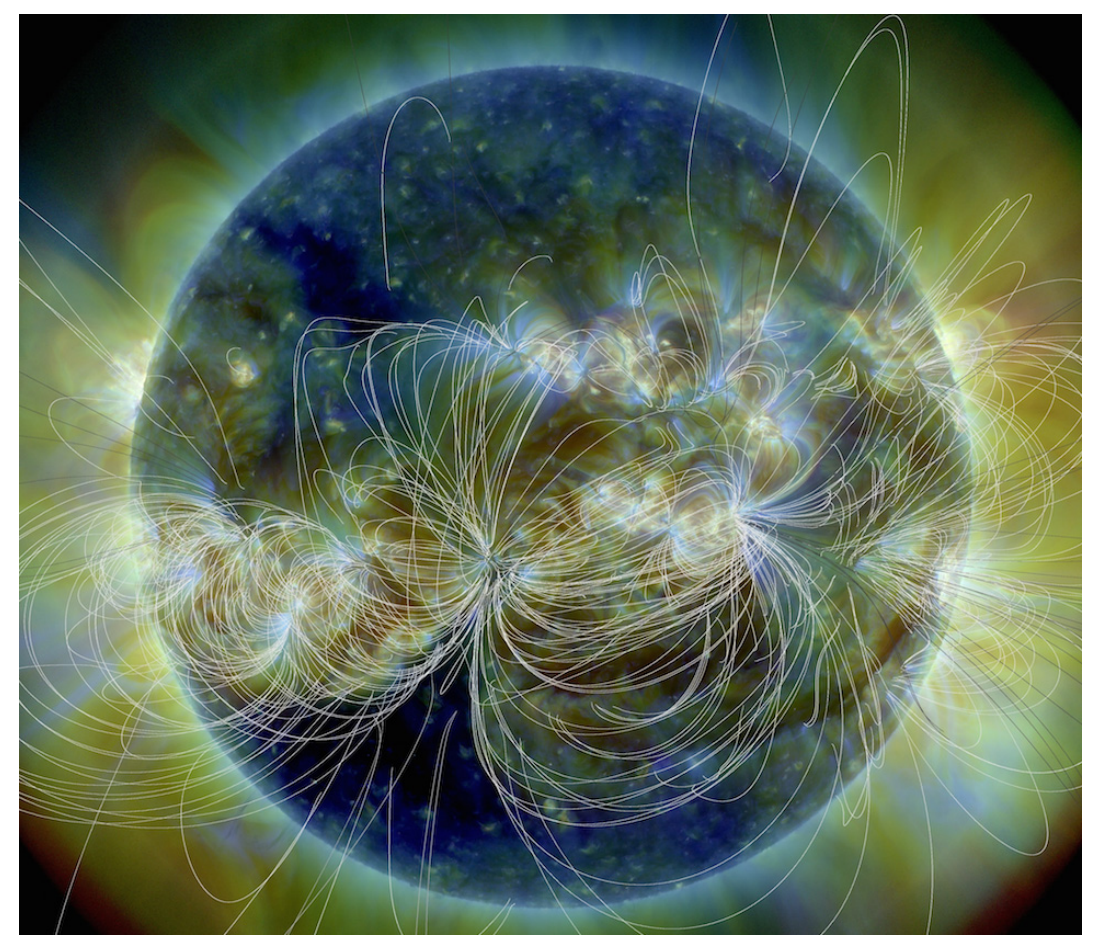

Figure 4: Magnetic field lines extrapolated from optical magnetogram superposed on a composite SDO/AIA (211 A, $193 \AA, 171 \AA)$ image, on 7 April 2014. Credit: NASA/LMSAL/SDO.

Implications of these results on the theory of coronal heating were discussed in Klimchuk (2000), but the conclusion was that none of the models alone is able to explain all observed prop- 
erties. Important information about the internal structuring of coronal loops comes from the joint analysis of the photospheric and coronal magnetic field (Figure 4). TRACE loops are quite symmetric and their cross-section is constant to a good degree of approximation, at variance from the prediction by linear force-free extrapolation on SoHO/MDI data (López Fuentes et al., 2006). The magnetic field lines starting from the same footpoint can diverge to different end footpoints, and thus be very complicated with strong tangling of the magnetic flux strands driven by the photospheric convection. This is not observed at high-resolution in the quiescent corona, possibly because of braiding-induced interchange reconnection of the magnetic field (Schrijver, 2007). Other approaches address also the density values and stratification, and explain the evidence with a combination of high plasma density within the structures, which greatly increases the emissivity of the structures, and geometric effects that attenuate the apparent brightness of the feature at low altitudes (DeForest, 2007). More recent MHD modeling finds that the temperature distribution across the loop naturally leads to the appearance of constant cross-section in EUV band (Peter and Bingert, 2012). Another model shows that the apparent constant loop cross section is a result of the non-circular shape of the loops (Malanushenko and Schrijver, 2013).

An alternative analysis of the magnetic field at the footpoints of hot and cool loops showed that the magnetic filling factor is lower in hot loops (0.05-0.3 out of sunspots) than in warm loops (0.2-0.6) (Katsukawa and Tsuneta, 2005). The multispectral analysis of solar EUV images has showed the possibility of separating the different solar structures from a linear combination of images to emphasise the structures (Dudok de Wit and Auchère, 2007).

The general impression is that, although moderate deviations from the ideal circular shape with constant cross-section are often observed, they probably do not affect the description of loops with a simplified geometry.

\subsubsection{Fine structuring}

It has been long claimed that coronal loops consist of bundles of thin strands, to scales below the instrumental resolution (e.g., Gómez et al., 1993). The issue of fine loop structure is of critical importance because it constrains the elementary processes that determine the loop ignition. The task to investigate this substructuring is not easy. Studies based both on models and on analysis of observations independently suggest that elementary loop components should be very fine with typical cross-sections of the strands on the order of 10-100 km (Beveridge et al., 2003; Cargill and Klimchuk, 2004; Vekstein, 2009). First limited evidence of fine structuring was the low filling factor inferred for loops observed with NIXT (Di Matteo et al., 1999, see Section 3.3.2). The high spatial resolution achieved by the TRACE normal-incidence telescope allowed to address the transverse structure of the imaged coronal loops. EUV images visibly show that coronal loops are substructured (Figure 5).

There were some early attempts to study the structure along the single strands in TRACE observations (Testa et al., 2002). Later, it was shown that in many cases, hot loop structures observed in active regions with the Yohkoh/SXT $(T>3 \mathrm{MK})$ are not exactly co-spatial with warm structures $(T \sim 1-2 \mathrm{MK})$ observed in the EUV bands $(195 \AA)$ of the SoHO/EIT, nor they cool down to become visible with EIT (Nagata et al., 2003; Schmieder et al., 2004). In another study, hot monolithic loops visible with the Yohkoh/SXT were instead resolved as stranded cooler structures with TRACE at later times (Winebarger and Warren, 2005), although the large time delay (1 to 3 hours) is hardly compatible with the cooling time from SXT to TRACE sensitivity. More systematic studies of TRACE images proposed that about $10 \%$ of the positions across loops can be fitted with an isothermal model, indicating coherent loop components about $2000 \mathrm{~km}$ wide (Aschwanden and Nightingale, 2005; Aschwanden et al., 2007).

More recent studies have found other indirect evidence for loop fine structuring. For instance, the different fuzziness measured in spectral lines forming at different coronal temperatures (Tri- 


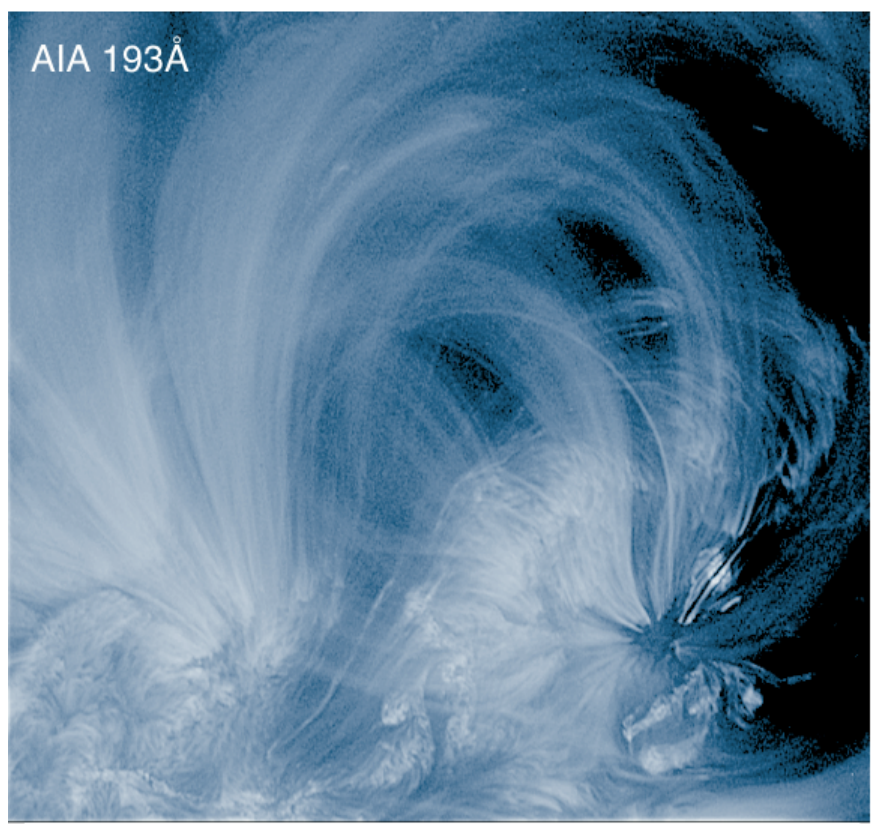

Figure 5: SDO/AIA (193 A channel) image of finely-structured coronal loops (14 October 2011, 22:56 UT). The image was treated with a Gaussian sharpening filter with a radius of 3 pixels. Image reproduced with permission from Brooks et al. (2012), copyright by AAS.

pathi et al., 2009) has been modeled with loops made of tens of independently pulse-heated strands (Guarrasi et al., 2010; Reale et al., 2011). Other indirect evidence might come from optical observations of relatively dense and cool $(\sim 7000 \mathrm{~K})$ downfalling elongated blobs above the solar limb, the so-called "coronal rain" (Antolin and Rouppe van der Voort, 2012), with widths of the order of $500 \mathrm{~km}$. Upflows in even narrower channels $(\sim 100 \mathrm{~km})$ were resolved in the optical band, correlating with brightenings in SDO/AIA observations (Ji et al., 2012).

New direct measurements of the loop fine structure are stimulated by the new observations with normal-incidence telescopes (SDO/AIA, Hi-C) and by indications that the elementary components of most loops might be small scale and close to be resolved (Brooks et al., 2012). Some quantitative constraints come from the very high spatial resolution of the Hi-C sounding rocket, with crosssections that range between about $300 \mathrm{~km}$ (Brooks et al., 2013) to over $1000 \mathrm{~km}$ (Peter et al., 2013) for long loops, with possibility of further substructuring well below $100 \mathrm{~km}$.

Evidence for fine structure can come also from studies of thermal structuring; in general, an emission measure distribution extending over a broad temperature range along the line of sight is retained to be a signature of the presence of multi-stranded structure, because of the coexistence of many thermal components. See Section 3.3 for more details.

In general, there is converging evidence for fine loop structuring to scales on the order of $100 \mathrm{~km}$ or below. Ultimate information could be provided by higher resolution future instruments.

\subsection{Diagnostics and thermal structuring}

The investigation of the thermal structure of coronal loops is very important for their exhaustive physical comprehension and to understand the underlying heating mechanisms. For instance, one of the classifications outlined above is based on the loop thermal regime, and, we remark, it is debated whether the classification indicates a real physical difference. 
Diagnostics of temperature are not trivial in the corona. No direct measurements are available. Since the plasma is optically thin, we receive information integrated on all the plasma column along the line of sight. The problem is to separate the distinct contributing thermal components and reconstruct the detailed thermal structure along the line of sight. However, even the determination of global and average values deserves great attention. Exploratory measurements have shown that the ion temperature can be different from the electron temperature in the quiet corona (Landi, 2007).

Moderate diagnostic power is allowed by imaging instruments, by means of multifilter observations. Filter ratio maps (Figure 6) provide information about the spatial distribution of temperature and emission measure (e.g., Vaiana et al., 1973). The emission of an optically thin isothermal plasma as measured in a $j$-th filter passband is:

$$
I_{j}=E M G_{j}(T),
$$

where $T$ is the temperature and $E M$ is the emission measure, defined as

$$
E M=\int_{V} n^{2} d V,
$$

where $n$ is the particle density, and $V$ the plasma volume. The ratio $R_{i j}$ of the emission in two different filters $i, j$ is then independent of the density, and only a function of the temperature:

$$
R_{i j}=\frac{I_{i}}{I_{j}}=\frac{G_{i}(T)}{G_{j}(T)} .
$$

The inversion of this relationship provides a value of temperature, based on the isothermal assumption.

The limitations of this method are substantial. In particular, one filter ratio value provides one temperature value for each pixel; this is a reliable measurement, within experimental errors, as long as the assumption of isothermal plasma approximately holds for the plasma column in the pixel along the line of sight. If the plasma is considerably multithermal, the temperature value is an average weighted for the instrumental response. Since the response is a highly nonlinear function of the emitting plasma temperature, it is not trivial to interpret the related maps correctly. In addition, it is fundamental to know the instrument response with high precision, in order to avoid systematic errors, which propagate dangerously when filter ratios are evaluated. In this respect, broadband filters provide robust thermal diagnostics, because they are weakly dependent on the details of the atomic physics models, e.g., on the presence of unknown or not well-known spectral lines, on the choice of element abundances. Narrowband filters can show non-unique dependencies of filter ratio values on temperature (e.g., Patsourakos and Klimchuk, 2007), due to the presence of several important spectral lines in the bands, but a more general problem can be the bias to detect narrow ranges of temperatures forced by the specific instrument characteristics (Weber et al., 2005). This problem can be important especially when the distribution of the emission measure along the line of sight is not simple and highly nonlinear (e.g., Reale et al., 2009a). New methods for thermal diagnostics with narrow band instruments have been proposed (Dudok de Wit et al., 2013).

The problem of diagnostics of loop plasma from filter ratios, and, more in general, the whole analysis of loop observations, are made even more difficult by the invariable presence of other structures intersecting along the line of sight. A uniform diffuse background emission also affects the temperature diagnostics, by adding systematic offsets that alter the filter ratio values. The task of subtracting this "background emission" from the measured emission is non-trivial and can seriously bias the results of the whole analysis. This problem emerged dramatically when the analysis of the same large loop structure observed with Yohkoh/SXT on the solar limb led to three 
different results depending mostly on the different ways to treat the background (Priest et al., 2000; Aschwanden et al., 2001; Reale, 2002a). The amount of background depends on the instrument characteristics, such as the passband and the point response function: it is most of the signal in TRACE UV filterbands, for instance, and its subtraction becomes a very delicate issue (e.g., Del Zanna and Mason, 2003; Reale and Ciaravella, 2006; Aschwanden et al., 2008; Terzo and Reale, 2010). The problem can be mitigated if one analyzes loops as far as possible isolated from other loops, but this is not easy, for instance, in active regions. If this is not the case, broadband filters may also include contamination from many structures at relatively different temperature and make the analysis of single loops harder. The problem of background subtraction in loop analysis has been addressed by several authors, who apply different subtraction ranging from simple offset, to emission in nearby pixels or subregions, to values interpolated between the loop sides, to whole images at times when the loop is no longer (or not yet) visible (Testa et al., 2002; Del Zanna and Mason, 2003; Schmelz et al., 2003; Aschwanden and Nightingale, 2005; Reale and Ciaravella, 2006; Aschwanden et al., 2008; Terzo and Reale, 2010).

More accurate diagnostics, although with less time and space resolution, are in principle provided by spectrometers and observations in temperature-sensitive spectral lines, which are being constantly improved to provide better and better spatial information. Early results from UV spectroscopy already recognized the link between transition region and coronal loops, for instance, from Skylab mission (Feldman et al., 1979; Mariska et al., 1980). There is a considerable effort to develop and update continuously the spectral codes and databases, in particular the CHIANTI spectral line database (Dere et al., 1997; Landi et al., 1999; Dere et al., 2001; Landi et al., 2006; Dere et al., 2009; Landi et al., 2012a; Del Zanna, 2012; Landi et al., 2013). Together with background subtraction, one major difficulty met by spectroscopic analysis is that, in the UV band, the density of lines is so high that they are often blended and, therefore, it is hard to separate the contribution of the single lines, especially the weak ones. Fine diagnostics, such as Doppler shifts and line broadening, can become very tricky in these conditions and results are subject to continuous revisitation and warnings from the specialized community. The problem of background subtraction is serious also for spectral data, because their lower spatial and temporal resolution determines the presence of more structures and, therefore, more thermal components, along the line of sight in the same spatial element.

Care should be paid also when assembling information from many spectral lines into a reconstruction of the global thermal structure along the line of sight. Methods are well-established (e.g., Gabriel and Jordan, 1975) and several approaches are available. The so-called method of the emission measure loci (Pottasch, 1963; Jordan et al., 1987; Landi et al., 2002) is able to tell whether plasma is isothermal of multithermal along the line of sight (Figure 7), but less able to add details. Detailed emission measure distributions can be obtained from differential emission measure (DEM) reconstruction methods (e.g., Brosius et al., 1996; Kashyap and Drake, 1998), but this is an ill-posed mathematical problem, and, therefore, results are not unique and are subject to systematic and unknown errors.

There is no wide convergence on the reconstruction of the temperature distribution of the emission measure along the line of sight. So several works have been devoted to develop new methods (e.g., Hannah and Kontar, 2012) and to investigate the limits and capabilities of the methods themselves. One important question is how far can we push the temperature resolution of the methods. We expect better resolution switching from narrow-band instruments with few channels (Weber et al., 2005) to spectrometers (Landi and Klimchuk, 2010; Landi et al., 2012b), but it is difficult to achieve a temperature resolution better than $\Delta \log T \approx 0.05$ (Landi et al., 2012b). Reconstructions from narrow-band data may suffer from biases that affect their reliability (Weber et al., 2005) and produce clustering (Guennou et al., 2012a,b). Not only the width and sensitivity of the spectral band is important, but also its position in the temperature range. It has been remarked that, with the available instruments, it is difficult to constrain emission from plasma at 


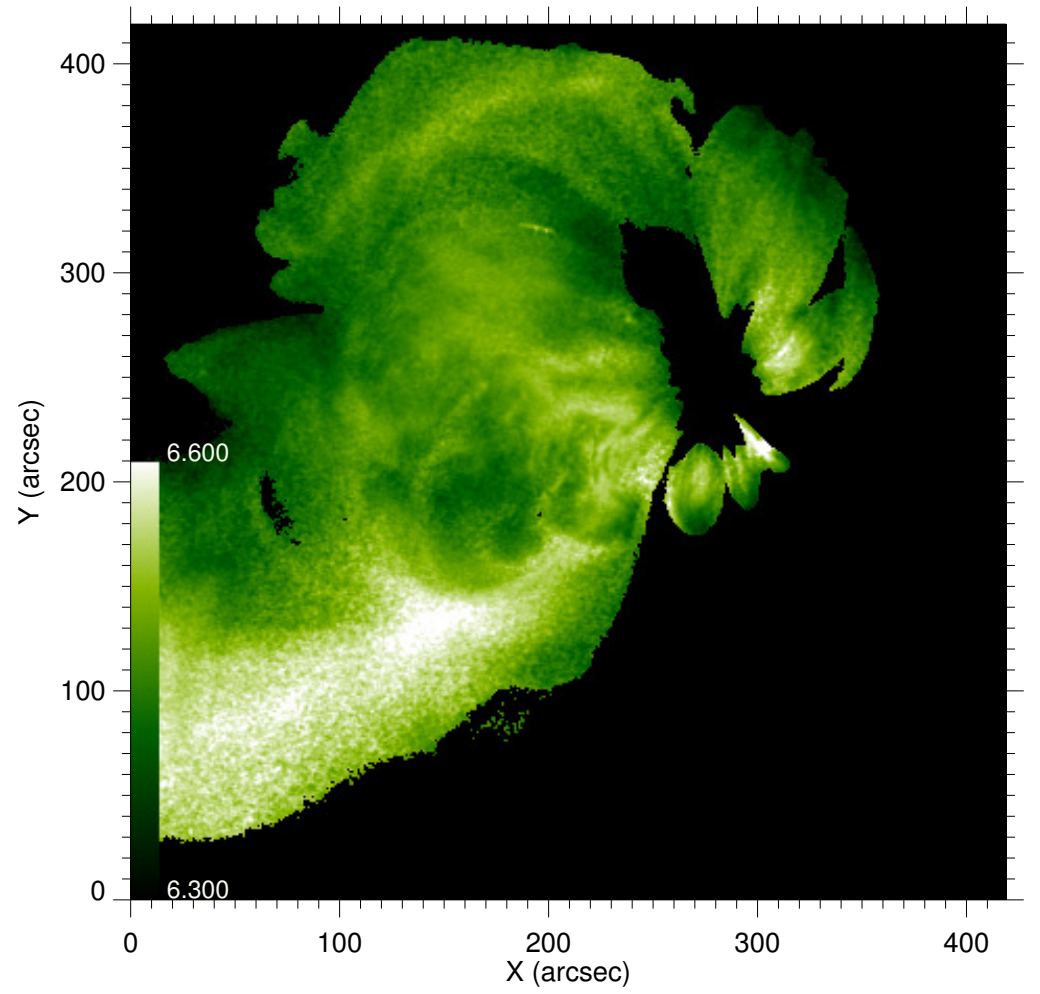

Figure 6: Temperature map of an active region obtained from the ratio of two images in different broadband filters with Hinode/XRT (12 November 2006, 12 UT).

temperature above about $6 \mathrm{MK}$ and emission measures less than $\sim 10^{27} \mathrm{~cm}^{-5}$ (Winebarger et al., 2012). Under some conditions, these limitations might be overcome, as described in Section 3.3.1. It is also useful to assess unknown uncertainties in the atomic data (Landi et al., 2012b). Allencompassing 3D MHD simulations allow to build a complete cycle from true values to observables back to reconstructed values and comparison with true values (Testa et al., 2012). It is important to test the combination of all possible uncertainties, the overall capability of the methods, indicating that a highly non-uniform density distribution along the line of sight can mislead the methods.

Forward modeling and simulations can be ways to escape from these problems, but they require non-trivial computational efforts and programming, and it is not always possible to provide accurate confidence levels. All these approaches are constantly improved and, probably, the best way to proceed is to combine different approaches and multiband observations and to finally obtain a global consistency.

Interesting issues come from global thermal analysis of the confined solar corona. The analysis of full-disk data from spatially-unresolved SphinX spectral data at the solar minimum of 2009 shows temperatures of 1.7-1.9 MK and emission measures between $4 \times 10^{47} \mathrm{~cm}^{-3}$ and $1.1 \times 10^{48} \mathrm{~cm}^{-3}$. Most of the emission comes from the large-scale corona rather than localized and bright structures, e.g., bright points (Sylwester et al., 2012).

In addition to the problems intrinsic to diagnostic techniques, we have to consider that loops appear to have different properties in different bands, as mentioned in Section 3.1.1. It is still debated whether such differences originate from an observational bias due to the instruments or from intrinsic physical differences, or both. In view of this uncertainty, in the following we will make a distinction between hot and warm loops, which will generally correspond to loops observed 
in the X-ray (and hot UV lines, e.g., Yohkoh/SXT, Hinode/XRT, SoHO/CDS Fe XVI line) and in the UV band (e.g., SoHO/EIT, TRACE), respectively. Cool loops are also observed in the UV band. The boundary between hot and warm loops is, of course, not sharp, and it is not even clear whether they are aspects of the same basic structure, or they really are physically different and are heated differently (see also Section 3.3.3). We will now devote our attention to the comparison between hot and warm loops.

\subsubsection{Hot loops}

After the pioneering analyses driven by the Skylab X-ray instruments (Section 2), Yohkoh/SXT allowed to conduct large-scale studies on the thermal and structure diagnostics of hot loops, mostly located in active region, and the comparison with other instruments, for instance on-board SoHO, allowed to obtain important cross-checks and additional information. Filter ratio maps of flaring loops were shown early after the mission launch (Tsuneta et al., 1992).

Systematic measurements of temperature, pressure, and length of tens of both quiescent and active region coronal loops were conducted on Yohkoh observations in the 1990s (Porter and Klimchuk, 1995) using the filter ratio method. Steady and isolated loops were selected with a length in a decade between $5 \times 10^{9}<2 L<5 \times 10^{10} \mathrm{~cm}$. Their average temperature was high, ranging in a decade $(2<T<30 \mathrm{MK})$, with a mean of about $6 \mathrm{MK}$ and with large uncertainties in the hot tail of the distribution. Pressure was estimated to span over two decades $\left(0.1<p<20 \mathrm{dyne} \mathrm{cm}^{-2}\right)$. The temperature and length were uncorrelated, while the pressure was found overall to vary inversely with the length (as overall expected for a thermally homogeneous sample from loop scaling laws, see Section 4.1.1), allowing to constrain the dependence of the magnetic field intensity on the loop length (Klimchuk and Porter, 1995), and the data uncertainties (Klimchuk and Gary, 1995). Another systematic analysis on another sample of Yohkoh loops in active regions (Kano and Tsuneta, 1995) confirmed some of the correlations in Porter and Klimchuk (1995), but also found a correlation between the loop length and the temperature, and deviations from RTV scaling laws (Section 4.1.1). The correlations might depend on the loop sample, as a single scaling law links three parameters. Very hot Yohkoh/SXT loops were found to have short lifetimes (less than few hours), and often to exhibit cusps (Yoshida and Tsuneta, 1996).

Density diagnostics through density-sensitive line ratio led to measure directly density values in active regions (e.g., Doschek et al., 2007). The hot core of the active region is densest, with values as high as $10^{10.5} \mathrm{~cm}^{-3}$. Density in active regions has been measured also using Fe lines, with values in the range $8.5 \leq \log \left(N_{\mathrm{e}} / \mathrm{cm}^{-3}\right) \leq 11.0$ (Young et al., 2009), or in a smaller range (from $10^{8.5}$ to $10^{9.5} \mathrm{~cm}^{-3}$ ) (Watanabe et al., 2009). Diagnostics of high density structures are also possible from the analysis of absorbed structures observed with narrow-band imagers (e.g., Landi and Reale, 2013).

A big effort has been devoted to the possible detection of hot plasma outside of evident flares. This would be a conclusive evidence of the presence of impulsive heating mechanisms in coronal loops (e.g., Klimchuk, 2006, see Section 4.4). Hinode instruments appear to be able to provide new interesting contributions to this topic. The analysis of spectroscopic observations of hot lines in solar active regions from Hinode/EIS allows to construct emission measure distributions in the 1-5 MK temperature range, and shows that the distributions are flat or slowly increasing up to approximately $3 \mathrm{MK}$ and then fall off rapidly at higher temperatures (Patsourakos and Klimchuk, 2009). Emission from very hot lines has been early found in other Hinode/EIS observations, and in particular from the CaXVII at $192.858 \AA$, formed near a temperature of $6 \times 10^{6} \mathrm{~K}$, in active regions (Ko et al., 2009).

Thanks to its multifilter observations, also Hinode/XRT is providing useful information about the thermal structure of the bright X-ray corona. Temperature maps derived with combined filter ratios show fine structuring to the limit of the instrument resolution and evidence of multithermal 
components (Reale et al., 2007), as complemented by TRACE images. Observations including flare filters show evidence of a hot component in active regions outside of flares (Schmelz et al., 2009), and data in the medium thickness filters appear to constrain better this component of hot plasma as widespread, although minor, and peaking around $\log T \sim 6.8-6.9$, with a tail above $10 \mathrm{MK}$ (Reale et al., 2009b). Further support comes from RHESSI data (Reale et al., 2009a; McTiernan, 2009).

Further evidence for minor components of hot plasma in non-flaring active regions have been found from various other instruments. Analysis in the waveband 3.3-6.1 $\AA$ and $280-330 \AA$ with the RESIK and SPIRIT instruments, respectively, confirm the presence of a $0.1 \% \sim 10 \mathrm{MK}$ component at various activity levels (Sylwester et al., 2010; Shestov et al., 2010). Low-resolution SphinX spectra integrated on 17 days in the $2-10 \AA$ band still show a small but highly-significant component at about $7 \mathrm{MK}$ from active regions outside of microflares (Miceli et al., 2012). The separation of the hot from the cool components in the SDO/AIA $94 \AA$ channel indicates finelystructured Fe XVIII line emission in the core of bright active regions (Reale et al., 2011). This filamented emission at high temperature has been previously predicted with a model of multistranded pulse-heated loops (Guarrasi et al., 2010). The emission from hot emission lines (Ca XVII and FeXVIII) has been confirmed from simultaneous observations with SDO/AIA and with the Hinode/EIS spectrometer (Testa and Reale, 2012; Teriaca et al., 2012). However, while it has been proposed that AIA imaging observations of the solar corona can be used to track hot plasma (6-8 MK), it has been questioned that such emission is really at the temperature of the line sensitivity peak (Teriaca et al., 2012). Other analysis of a limb active region with EUV spectral data from Hinode/EIS does not find evidence for plasma at temperature $\log T>7$ (O'Dwyer et al., 2011) and puts an upper limit on the same track as remarked by Winebarger et al. (2012). So a final conclusion on this topic is still to be reached. There is some evidence that the amount of high-temperature plasma might correlate with the intensity of the active region magnetic fields because of increasing frequency of energy release (Warren et al., 2012).

\subsubsection{Comparison of hot and warm loops}

Before the SoHO/EIT and TRACE observations, warm loops had been imaged in a similar spectral band and with similar optics by the rocket NIXT mission (see Section 2). Studies of NIXT loops including the comparison with hydrostatic loop models (Section 4.1.1) pointed out that bright spots also visible in $\mathrm{H} \alpha$ band were the footpoints of hot high-pressure loops (Peres et al., 1994). This result was confirmed by the comparison of the temperature structure obtained from Yohkoh with NIXT data (Yoshida et al., 1995, see also Section 3.3.3).

Another comparison of loops imaged with NIXT and Yohkoh/SXT showed that the compact loop structures (length $\sim 10^{9} \mathrm{~cm}$ ) have a good general morphological correspondence, while larger scale NIXT loops $\left(\sim 10^{10} \mathrm{~cm}\right)$ have no obvious SXT counterpart (Di Matteo et al., 1999). Comparison with static loop models (see Section 4.1.1) allowed to derive estimates of the loop filling factors, important for the loop fine structure (Section 3.2.2). In the NIXT band, the filling factor of short loops was found to be very low $\left(10^{-3}-10^{-2}\right)$, but of the order of unity in the SXT band and for the largest structure. Simultaneous SoHO and Yohkoh observations of a small solar active region suggested a volume filling factor decreasing with increasing density and possible differences between emitting material in active regions and the quiet Sun (Griffiths et al., 2000). Other measurements of the filling factor come from density sensitive lines and images in the EUV band. For bright points the plasma-filling factor has been found to vary from $3 \times 10^{-3}$ to 0.3 with a median value of 0.04 , which may indicate considerable subresolution structure, or the presence of a single completely-filled unresolved loop with subarcsec width (Dere, 2008, 2009).

Some similarity between loops observed in the TRACE EUV band and Yohkoh X-ray band was found in outer active region loops (Nitta, 2000) and interpreted as evidence of loops with a 
broad range of temperatures. Core loops were instead observed only in the X-rays and found to be variable, indicating that probably they are not steady.

Density and temperatures in two active regions were accurately determined from SoHO-CDS observations (Mason et al., 1999) and it was confirmed quantitatively that the AR cores are hotter than larger loop structures extending above the limb. The analysis of a single loop observed on the solar limb with SoHO/CDS showed a bias to obtain flat temperature distributions along the loop from ratios of single lines or narrow band filters (TRACE), while a careful DEM reconstruction at selected points along the same loop was inconsistent with isothermal plasma, both across and along the loop (Schmelz et al., 2001). A whole line of works started from this analysis reconsidering and questioning the basic validity of the temperature diagnostics with TRACE and emphasizing the importance of the background subtraction, but also the need to obtain accurate spectral data (Schmelz, 2002; Martens et al., 2002; Aschwanden et al., 2002; Schmelz et al., 2003). Similar results but different conclusions were reached after the analysis of a loop observed with SoHO, invoking a non-constant cross-section to explain the evidence of isothermal loop (Landi and Landini, 2004; Landi and Feldman, 2004). On the other hand, evidence of non-uniform temperature along loops observed with TRACE was also found (Del Zanna and Mason, 2003; Reale and Ciaravella, 2006), emphasizing that the temperature diagnostic with narrow band instruments is a delicate issue.

An interesting debate focussed on the question whether the loops observed with TRACE and CDS have a uniform transverse thermal distribution, i.e., a narrow DEM, or a multi-thermal distribution, i.e., a wide DEM that may group together warm and hot loops. Although tackled from a different perspective, this question also concerns the fine longitudinal structuring of the loops and of their heating and is therefore strictly connected to the subject of Sections 3.2.2 and 4.4. A loop imaged by TRACE was found to be isothermal (with temperatures below 1 MK) along the line of sight from diagnostics of spectral lines obtained with SoHO/CDS (Del Zanna and Mason, 2003). The distribution across another loop observed on the limb with SoHO/CDS was found multi-thermal, with a DEM reconstruction and a careful analysis of background subtraction (Schmelz et al., 2005). From the comparison with the isothermal structure of hot loops derived from CDS data (Di Giorgio et al., 2003; Landi and Landini, 2004) and a systematic inspection of the CDS atlas, the conclusion was that there might be two different classes of loops, multi-thermal and isothermal (Figure 7).

Comparative studies of active region loops in the transition region and the corona (UgarteUrra et al., 2009) observed with Hinode seem to point out the presence of two dominant loop populations, i.e., core multitemperature loops that undergo a continuous process of heating and cooling in the full observed temperature range $0.4-2.5 \mathrm{MK}$ shown by the X-Ray Telescope, and peripheral loops that evolve mostly in the temperature range $0.4-1.3 \mathrm{MK}$.

Multiband observations are able to provide more information and constraints. The analysis of an isolated loop in a time-resolved observation in several spectral bands, namely three TRACE UV filters, one Yohkoh/SXT filter, two rasters taken with SoHO/CDS in twelve relevant lines $(5.4 \leq \log T \leq 6.4)$, supported a coherent scenario across the different bands and instruments, i.e., a globally cooling loop and the presence of thermal structuring (Reale and Ciaravella, 2006). The analysis overall indicated that the loop analysis can be easily affected by a variety of instrumental biases and uncertainties, for instance due to rough background subtraction. The fact that the loop that could be well analyzed across several bands and lines is a cooling loop may not be by chance (see end of Section 3.3.3).

SoHO spectrometric data have contributed to investigate the loop thermal structure for a long time. From a differential emission measure (DEM) analysis with a forward-folding technique on SoHO/CDS data, some loops were found to be isothermal and others to have a broad DEM (Schmelz et al., 2007, 2008). Three distinct isothermal components, reminiscent of coronal hole, quiet-Sun, and active region plasmas, were found from the analysis of an active region spectrum observed by the SoHO/SUMER (Landi and Feldman, 2008).

Living Reviews in Solar Physics

http://www. livingreviews.org/lrsp-2014-4 

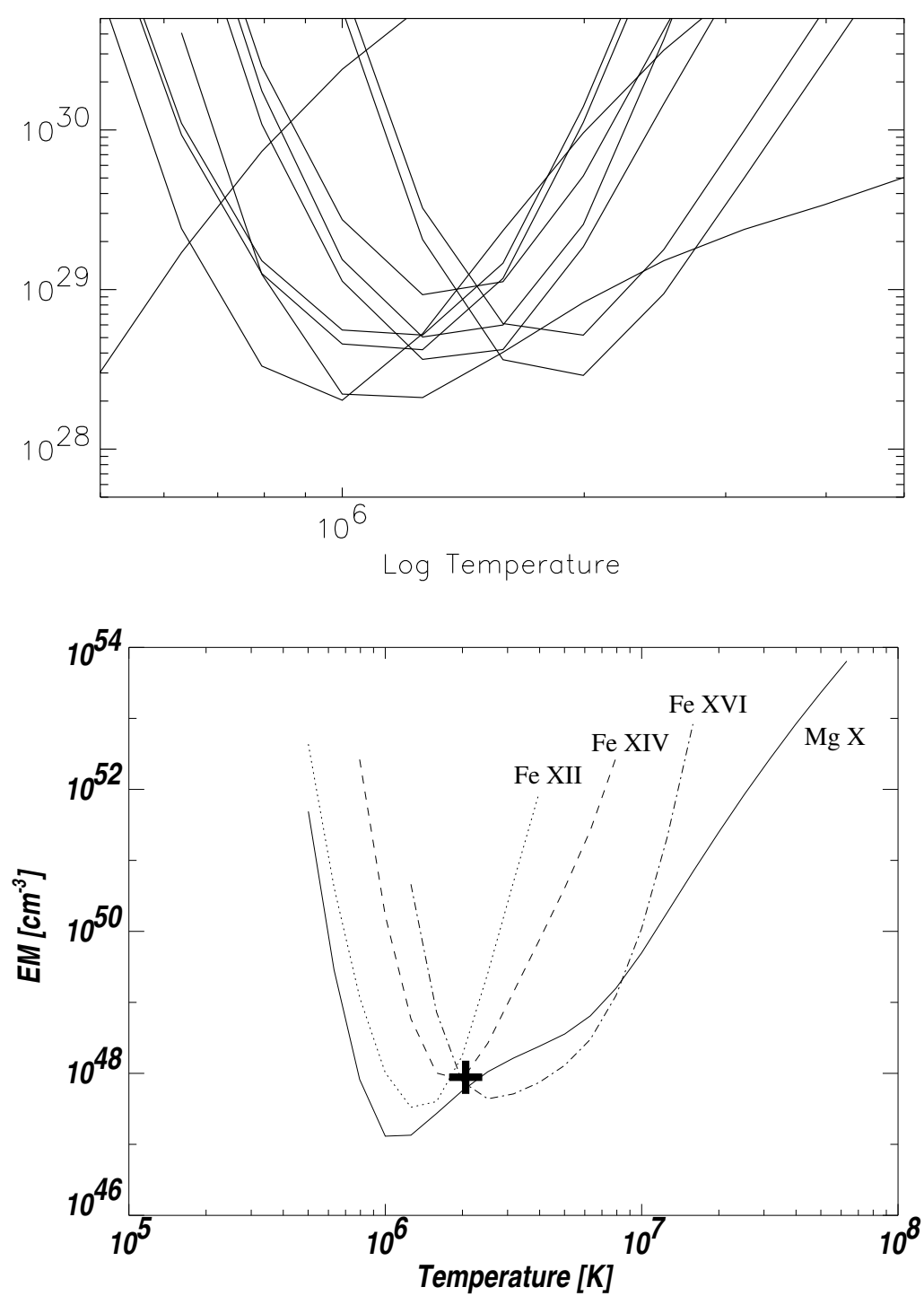

Figure 7: EM-loci plots for two different loop systems, one showing a multi-thermal structure (top, from Schmelz et al., 2005, reproduced with permission, copyright by AAS), the other an almost isothermal one (bottom, from Di Giorgio et al., 2003, reproduced with permission, copyright by ESO). The EM is per unit area in the top panel. 
Hinode/EIS observations of active region loops show that contrasted structures in cooler lines ( $1 \mathrm{MK})$ become fuzzy at higher temperatures ( $2 \mathrm{MK}$, Tripathi et al., 2009), Figure 8), as already pointed out by Brickhouse and Schmelz (2006). This issue is addressed by multi-thread loop modeling (Guarrasi et al., 2010).

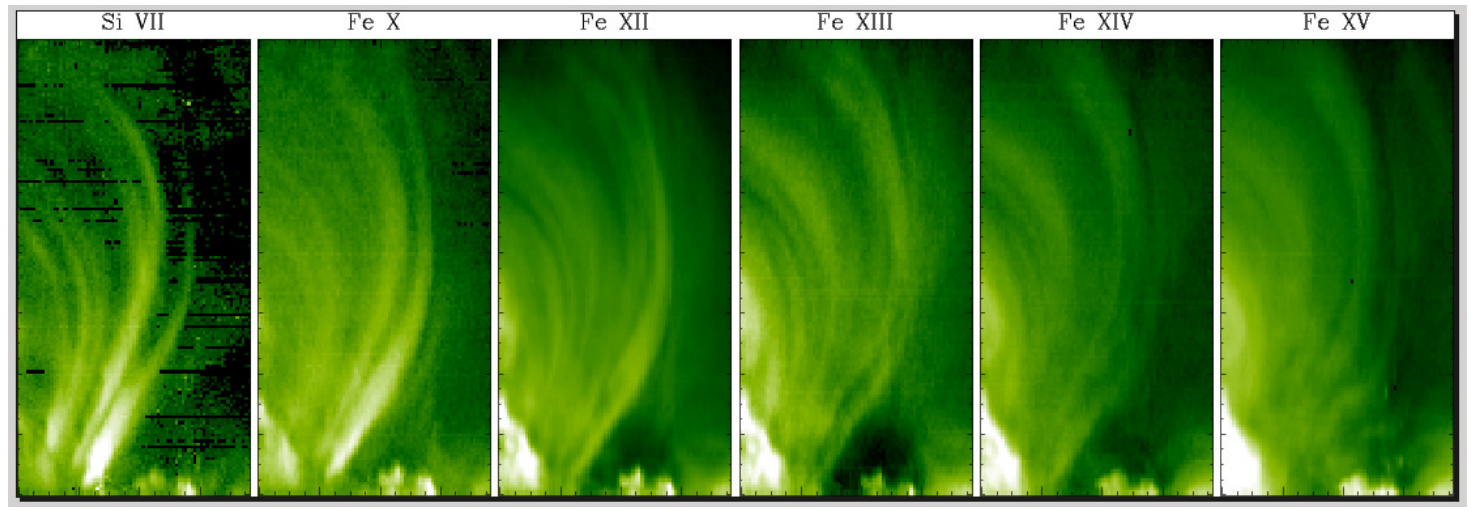

Figure 8: Loop system observed in several EUV spectral lines with Hinode/EIS (19 May 2007, 11:4116:35 UT). The loops become less and less contrasted, i.e., fuzzier and fuzzier, at higher and higher temperature. Courtesy of D. Tripathi.

Intensive efforts have been devoted to the analysis of the cool side of the emission measure distribution of hot loops, using data from EUV spectrometers. There is evidence for dynamic structure of active regions with frequent condensations inside (Tripathi et al., 2010) and for steep distributions (Tripathi et al., 2011). Analysis of Hinode and SDO observations were used to reconstruct the emission measure over a broad temperature range (Warren et al., 2011). Although affected by large uncertainties, it was found that at the apex of high-temperature loops the emission measure distribution has a relatively sharp peak around 4 MK.

\subsubsection{Warm loops}

The TRACE mission opened new intriguing questions because the data showed new features, e.g., stranded bright structures mostly localized in active regions, name "the moss", and because the narrow band filters offered some limited thermal diagnostics, but not easy to interpret. Reliable temperatures are in fact found in a very narrow range, and many coronal loops are found to be isothermal in that range.

As mentioned in Section 3.3.2, first loop diagnostics with normal-incidence telescopes were obtained from data collected with NIXT (Peres et al., 1994). The bright spots with H $\alpha$ counterparts were identified with the footpoints of high pressure loops, invisible with NIXT because not sensitive to plasma hotter than $1 \mathrm{MK}$. They have been later addressed as the moss in the TRACE images, which undergo the same effect, and their interactions with the underlying chromospheric structures began to be studied (Berger et al., 1999; Fletcher and De Pontieu, 1999). Comparison of SoHO/CDS and TRACE observations led to establish that the plasma responsible for the moss emission has a temperature range of about $1 \mathrm{MK}$ and is associated with hot loops at $1-2 \mathrm{MK}$, with a volume filling factor of order 0.1 (Fletcher and De Pontieu, 1999). It was also found that the path along which the emission originates is of order $1000 \mathrm{~km}$ long. According to an analytical loop model, a filling factor of about 0.1 is in agreement with the hypothesis of moss emission from the legs of $3 \mathrm{MK}$ loops (Martens et al., 2000). The electron density estimated in specific regions in the active region moss decreases with increasing temperature (Tripathi et al., 2008). The density within the moss region was highest at $\log T=5.8-6.1$, with a value around $10^{10} \mathrm{~cm}^{-3}$. 
As for temperature diagnostics with narrow band filters, loops soon appeared to be mostly isothermal with ratios of TRACE filters (Lenz et al., 1999; Aschwanden et al., 2000). Is this a new class of loops? Equivalent SoHO/EIT filter ratios provided analogous results (Aschwanden et al., 1999b). This evidence is intriguing and many investigations have addressed it (see also Section 3.3.2). From the diagnostic perspective, DEM reconstruction along the line of sight from spectral SoHO/CDS data and synthesized EIT count rates led to almost uniform temperatures along the loop, pointing again to an instrumental bias (Schmelz et al., 2001). Simple modeling confirmed that, provided they are flat, i.e., top-hat-shaped, even broad DEMs along the line of sight produce constant TRACE filter ratio values (Weber et al., 2005). On the other hand, the DEM obtained from spectrometers and from multi-wideband imagers data is most probably neither isothermal nor broad and flat, instead peaked with components extending both to low and high temperatures (e.g., Peres et al., 2000; Reale et al., 2009a). The critical point becomes the DEM width and its range of variation.

Using combinations of data from three filters, instead of two, does not seem to provide more information in the general case for warm loops (Schmelz et al., 2007), but the cross-field thermal structure of a sample of loops was found to be compatible with multithermal plasma with significant emission measure throughout the range 1-3 MK (Patsourakos and Klimchuk, 2007). With a combination of TRACE filter ratios, emission measure loci, and two methods of differential emission measure analysis, a few loops were found either isothermal or multithermal (Schmelz et al., 2009). This might not be a contradiction, in view of the presence of at least three possible conditions of warm loops, as discussed at the end of this section. Along a coronal loop in an active region on the solar limb, while TRACE double filter ratios led to temperatures between 1.0 and $1.3 \mathrm{MK}$, the emission measure loci from CDS data were consistent with a line-of-sight isothermal structure which increases in temperature from $\sim 1.20$ to $1.75 \mathrm{MK}$ along the loop, in contrast with the nearby multithermal background (Noglik et al., 2008).

Another puzzling issue, certainly linked to the loop isothermal appearance, is that warm loops are often diagnosed to be overdense with respect to the equilibrium values predicted by loop scaling laws (Lenz et al., 1999; Winebarger et al., 2003a, Section 4.1.1). To explain both these pieces of evidence, several authors claimed that the loops cannot be at equilibrium and that they must be filamented and cooling from a hotter state, probably continuously subject to heating episodes (nanoflares, Warren et al., 2002, 2003, Sections 4.2 and 4.4). Other authors proposed that part of the effect might be due to inaccurate background subtraction (Del Zanna and Mason, 2003).

The Hinode mission has stimulated extensive analyses of warm coronal structures, mostly based on its high quality EIS spectral data. Modeling observations of coronal moss with Hinode/EIS confirmed that the moss intensities predicted by steady, uniformly heated loop models are too intense relative to the observations (Warren et al., 2008b). A nonuniform filling factor is required and must vary inversely with the loop pressure. Observations of active region loops with EIS indicate that isolated coronal loops that are bright in Fe XII generally have very narrow temperature distributions $\left(3 \times 10^{5} \mathrm{~K}\right)$, but are not properly isothermal and have a volumetric filling factors of approximately $10 \%$ (Warren et al., 2008a).

In a cooler regime $(4.15<\log T<5.45)$ observed in coordination by SoHO spectrometers and imagers, STEREO/EUVI, and Hinode/EIS, active region plasma at the limb has been found to cool down from a coronal hole status with temperatures in the $5.6<\log T<5.9$ range (Landi et al., 2009).

A loop reconstruction from STEREO data improves the background subtraction and recovers density and temperature distributions that are able to reproduce the total observed fluxes within $20 \%$ (Aschwanden et al., 2009), emission measure distributions not very different from those obtained from spectroscopic observations (Brosius et al., 1996), and is in agreement with other previous studies (Lenz et al., 1999; Winebarger et al., 2003a).

Some studies have been devoted specifically to fan loops departing from active regions and 
observed with Hinode and SDO. It was found that these loops are warm and their temperature distribution is generally narrow (Brooks et al., 2011).

More recently, accurate analysis of warm loops with comparison between EUV spectroscopy and multi-channel imaging with Hinode/EIS and SDO/AIA have put severe warnings on using the latter for DEM reconstruction (Del Zanna et al., 2011). Cool plasma at temperature $T<0.5 \mathrm{MK}$ might contribute considerably to the emission, especially at the loop footpoints, and question, for instance, the interpretation of upflows observed in the $171 \AA$ band as made of million degrees plasma (De Pontieu et al., 2011). Important results come from observations at very high spatial resolution $(0.2$ arcsec) with the Hi-C sounding rocket in the EUV $193 \AA$ band. There is evidence of magnetic braiding that indicates the occurrence of magnetic reconnection (Cirtain et al., 2013).

In summary, the current observational framework and loop analysis seems to indicate that for a coherent scenario warm loops are manifestations of at least three different loop conditions: i) in loops consisting of bundles of thin independently-heated strands, few cooling strands of steady hot X-ray loops might be detected as warm overdense loops in the UV band. These warm loops would coexist with hot loops and would show a multithermal emission measure distribution (Patsourakos and Klimchuk, 2007; Warren et al., 2008a; Tripathi et al., 2009); ii) we might have warm loops as an obvious result of a relatively low average heating input in the loop. These loops would be much less visible in the X-rays and, thus, would not be co-spatial with hot loops, and would also be much less multithermal (Di Giorgio et al., 2003; Landi and Feldman, 2004; Aschwanden and Nightingale, 2005; Noglik et al., 2008); iii) warm loops might be globally cooling from a status of hot X-ray loop (Reale and Ciaravella, 2006). These loops would also be overdense and co-spatial with hot loops but with a time shift of the X-ray and UV light curves, i.e., they would be bright in the $\mathrm{X}$-rays before they are in the UV band. Also these loops would have a relatively narrow thermal distribution along the line of sight. There is some indication that there might be fundamental differences in the heating regime and cadence between hot and warm loops (Warren et al., 2010a).

\subsection{Temporal analysis}

The solar corona is the site of a variety of transient phenomena. Coronal loops sometimes flare in active regions (see the review by Benz, 2008). However, most coronal loops are well-known to remain in a steady state for most of their life, much longer than the plasma characteristic cooling times (Rosner et al., 1978, see Section 4.1.1). This is taken as an indication that a heating mechanism must be on and steady long enough to bring the loop to an equilibrium condition, and keep it there for a long time. Nevertheless, the emission of coronal loops is found to vary significantly on various timescales, and the temporal analyses of coronal loop data have been used to obtain different kinds of information, and as a help to characterize the dynamics and heating mechanisms. The time variability of loop emission is generally not trivial to interpret. The problem is that the emission is very sensitive to density and less to temperature. Therefore, variations are not direct signatures of heating episodes, not even of local compressions, because the plasma is free to flow along the magnetic field lines. Variations must therefore be explained in the light of the evolution of the whole loop. This typically needs accurate modeling, or, at least, care must be paid to many relevant and concurrent effects.

Another important issue is the band in which we observe. The EUV bands of the normalincidence telescopes are quite narrow. Observations are then more sensitive to variations because cooling or heating plasma is seen to turn on and off rapidly as it crosses the band sensitivity. On the other hand, telescopes in the X-ray band detect hotter plasma which is expected to be more sensitive to heating and therefore to vary more promptly, but the bandwidths are large and do not take as much advantage of the temperature sensitivity as the narrow bands. Finally, spectroscopic observations are, in principle, very sensitive to temperature variations, because they observe single lines, but their time cadence is typically low and able to follow variations only on 
large timescales. Time analysis studies can be classified to address two main classes of phenomena: temporal variability of steady structures and single transient events, such as flare-like brightenings.

In spite of limited time coverage, the instrument S-054 on-board Skylab already allowed for early studies of variability of hot X-ray loops. Measured decay times showed evidence of continued evaporation of coronal plasma in slowly-decaying structures (Krieger, 1978). found timescales of moderate variability was found on the timescale of a few hours over a substantial steadiness for observations of active region loops in 2 MK lines such as Fe XV and Si XII (Sheeley Jr, 1980; Habbal et al., 1985). Continuous microflare activity $\left(10^{26}-10^{28} \mathrm{erg}\right)$ has been extensively detected in hard X-ray band $(20 \mathrm{keV})$ with non-thermal power-law spectra (Lin et al., 1984). Substantial (but non-flaring) temporal variability was found in active region loops observed with SMM in a few relatively hot X-ray lines $(\sim 5 \mathrm{MK})$ on timescales of some minutes (Haisch et al., 1988). Cooler loops (< 1 MK Foukal, 1976, see Section 3.5) were found to be more variable and dynamic (e.g., Kopp et al., 1985).

The high time coverage and resolution of Yohkoh triggered studies of brightenings on short timescales. The study of the interaction of differently bright hot loops showed, for instance, that X-ray bright points often involve loops considerably larger than the bright points themselves, and that they vary on timescales from minutes to hours (Strong et al., 1992). The analysis of a large set (142) of macroscopic transient X-ray brightenings indicated that they derive from the interaction of multiple loops at their footpoints (Shimizu et al., 1994). Some other more specific loop variations, e.g., the shrinkage of large-scale non-flare loops were also observed, and interpreted not as an apparent motion, but as a real contraction of coronal loops that brighten and then gradually cool down (Wang et al., 1997). Fine-scale motions and brightness variations of the emission were found on timescales of 1 minute or less, often with dark jets of chromospheric plasma seen in the wings of $\mathrm{H} \alpha$, and probably associated with the fine structure and dynamics of the upper transition region (Berger et al., 1999).

Loop variability was specifically studied in several UV spectral lines observed with SoHO/CDS for about 3 hours by Di Giorgio et al. (2003). In the hottest lines, within the limited time resolution of about $10 \mathrm{~min}$, a few brightenings of a hot loop $(\sim 2 \mathrm{MK})$ were detected but they are minor perturbations over a steadily high emission level. A cool $\operatorname{loop}(\log T \sim 5.3)$ was confirmed to be a transient structure living a few hours, and confining substantial flows (Section 3.5).

Variability analyses were conducted also on warm loops present in TRACE data. The brightening of a single coronal loop was analyzed in detail in an observation of more than 2 hours with a cadence of about $30 \mathrm{~s}$ (Reale et al., 2000a). The loop brightens from the footpoints to the top, allowing for detailed hydrodynamic modeling (Reale et al., 2000b, see also Section 4.4). Active region transient events, i.e., short-lived brightenings in small-scale loops, detected over a neutral line in a region of emerging flux were interpreted as reconnection events associated with flux emergence (Seaton et al., 2001). Apparent shrinking and expansion of brightening warm loops suggested heating and cooling of different concentric strands, leading to coronal rain visible in the $\mathrm{H} \alpha$ line (Shimojo et al., 2002, see also Section 3.2.2). Plasma condensations in hot and warm loops were detected also in the analysis of line intensity and velocity in temporal series data from SoHO/CDS (O'Shea et al., 2007). Antiochos et al. (2003) found no significant variability of the moss regions observed with TRACE. This has been taken as part of the evidence toward steady coronal heating in active region cores (Warren et al., 2010b, see Section 4.4).

The analysis of temporal series from various missions has been used to investigate the possible presence of continuous impulsive heating by nanoflares. The temporal evolution of hot coronal loops was studied in data taken with GOES Solar X-ray Imager (SXI), an instrument with moderate spatial resolution and spectral band similar to Yohkoh/SXT (López Fuentes et al., 2007). The durations and characteristic timescales of the emission rise, steady and decay phases were found to be much longer than the cooling time and indicate that the loop-averaged heating rate increases slowly, reaches a maintenance level, and then decreases slowly (Figure 9), not in contradiction 
with the early results of Skylab (Section 2). This slow evolution is taken as an indication of a single heating mechanism operating for the entire lifetime of the loop. If so, the timescale of the loop-averaged heating rate might be roughly proportional to the timescale of the observed intensity variations.

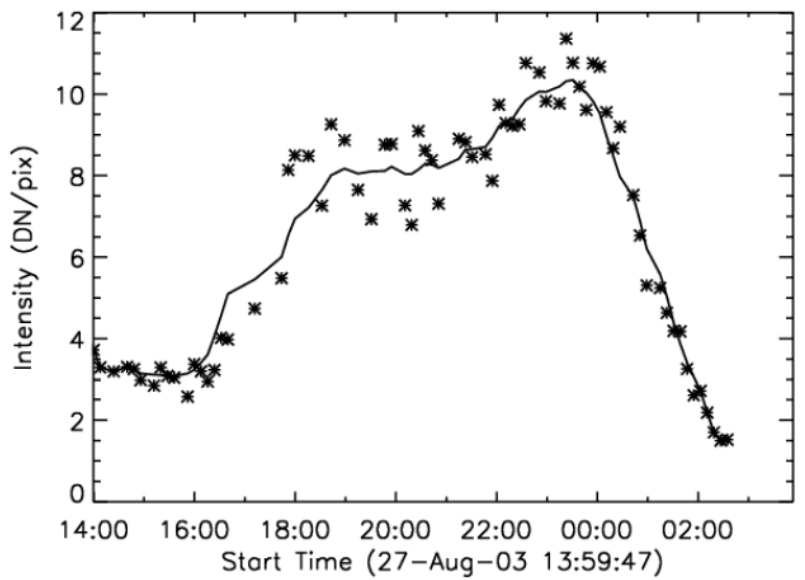

Figure 9: X-ray light curve observed with the SXI telescope on board GOES. The loop lifetime is much longer than the characteristic cooling times. Courtesy of J. A. Klimchuk and M. C. López Fuentes).

Joint TRACE and SoHO/CDS observations allowed to study temperature as a function of time in active region loops (Cirtain et al., 2007). In many locations along the loops, the emission measure loci were found consistent with an isothermal structure, but the results also indicated significant changes in the loop temperature (between 1 and $2 \mathrm{MK}$ ) over the $6 \mathrm{hr}$ observing period. This was interpreted as one more indication of multistranded loops, substructured below the resolution of the imager and of the spectrometer. Further support to fine structuring comes from the analysis of the auto-correlation functions in SXT and TRACE loop observations (Sakamoto et al., 2008). The duration of the intensity fluctuations for the hot SXT loops was found to be relatively short because of the significant photon noise, but that for the warm TRACE loops agrees well with the characteristic cooling timescale, thus supporting a continuous heating by impulsive nanoflares. The energy of nanoflares is estimated to be $10^{25} \mathrm{erg}$ for SXT loops and $10^{23} \mathrm{erg}$ for TRACE loops, and their occurrence rate about 0.4 and 30 nanoflares $\mathrm{s}^{-1}$, respectively.

Time series have been studied also on data taken with the Hinode mission. Hinode's Solar Optical Telescope (SOT) magnetograms and high-cadence EIS spectral data were used to distinguish hot, relatively steadily emitting warm coronal loops from isolated transient brightenings and to find that they are both associated with highly dynamic magnetic flux regions. Brightenings were confirmed in regions of flux collision and cancellation, while warm loops are generally rooted in magnetic field regions that are locally unipolar with unmixed flux (Brooks et al., 2008). It was suggested that the type of heating (transient vs. steady) is related to the structure of the magnetic field, and that the heating in transient events may be fundamentally different from that in warm coronal loops.

More recently, light curves in individual pixels have been investigated in the X-ray band to search for significant variability connected to variable heating. Although some pulses are detected, most of the emission in active region cores and loops has been found to be steady on the timescale of hours with fluctuations on the order of $15 \%$ and with no correlation between warm and hot emission (Warren et al., 2010b, 2011). Improving on previous studies (Sakamoto et al., 2008), 
high cadence observations with the Hinode/XRT have revealed that the distributions of intensity fluctuations have small but significant and systematic asymmetries. Part of this asymmetry has been explained through a tendency for exponentially decreasing intensity, i.e., the plasma has been cooling for most of the time (Terzo et al., 2011).

Loop light curves have been systematically analysed also in the EUV band. A systematic tendency has been found to have ordered time lags from channels sensitive to emission from hotter plasma to cooler plasma, that is also evidence for dominant cooling (Viall and Klimchuk, 2011, 2012). Light curves in the EUV band have been analysed also with a different approach: they have been compared to simulated ones obtained from sequences of random pulses with powerlaw distribution (Tajfirouze and Safari, 2012). Artificial neural network (ANN) was used for the comparison and it was found many that light curves are matched by those generated from events power-laws with a steep index $(\geq 2)$. While studying the long-term evolution of active regions on the large scale, it was found that active regions show less and less variability as they age (UgarteUrra and Warren, 2012), thus suggesting a qualitative change of heating frequency with time. At the other extreme of the smallest scales, the Hi-C observations show in some moss regions variability on timescales down to $\sim 15 \mathrm{~s}$, that may indicate the presence of heating pulses of comparable duration.

\subsection{Flows and waves}

Diagnosing the presence of significant flows in coronal loops is not an easy task. Apparently moving brightness variations may not be a conclusive evidence of plasma motion, since the same effect may be produced by the propagation of thermal fronts or waves. Conclusive evidence of plasma motion comes from measurements of Doppler shifts in relevant spectral lines. However, the detection of significant Doppler shifts requires several conditions to be fulfilled at the same time, e.g., significant component along the line of sight, amount of moving plasma larger than amount of static plasma, plasma motion comparable to typical line broadening effects.

In general, we can distinguish two main classes of mass bulk motions inside coronal loops: siphon flows, due to a pressure difference between the footpoints, and loop filling or draining, due to transient heating and subsequent cooling, respectively. Some other evidence of bulk motions, such as systematic redshifts in UV lines, has been difficult to interpret.

Siphon flows have been mainly invoked to explain motions in cool loops. The existence of cold loops has been known for a long time (Foukal, 1976, see Section 2) and SoHO has collected highquality data showing the presence of dynamic cool loops (Brekke et al., 1997). A well-identified detection was found in SoHO/SUMER data, i.e., a small loop showing a supersonic siphon-like flow (Teriaca et al., 2004) and in SoHO/CDS data (Di Giorgio et al., 2003).

Redshifts in transition region UV lines have been extensively observed on the solar disk (e.g., Doschek et al., 1976; Gebbie et al., 1981; Dere, 1982; Feldman et al., 1982; Klimchuk, 1987; Rottman et al., 1990; Brekke, 1993; Peter, 1999). Some mechanisms have been proposed to explain these redshifts: downward propagating acoustic waves (Hansteen, 1993), downdrafts driven by radiativelycooling condensations in the solar transition region (Reale et al., 1996, 1997b), nanoflares (Teriaca et al., 1999); the scenario is improving with the better and better definition of the observational framework. Downflows are systematically confirmed in lines at transition region temperatures $\left(\leq 0.5 \mathrm{MK}\right.$ ) from a few $\mathrm{km} \mathrm{s}^{-1}$ (Feldman et al., 2011) to a few tens $\mathrm{km} \mathrm{s}^{-1}$ (Chen and Ding, 2010; Ugarte-Urra and Warren, 2011). Moderate downflows have been detected at the boundary of active regions (Boutry et al., 2012). Redshifts between 5 and $15 \mathrm{~km} \mathrm{~s}^{-1}$ have been measured accurately from SoHO/SUMER data in three active regions with little spatial and temporal correlation (Winebarger et al., 2013).

Blueshifts in the transition region are also studied but not necessarily associated with coronal loops (e.g., Dere et al., 1986). More localized and transient episodes of high velocity outflows, 
named explosive events, have been observed in the transition lines such as C IV, formed at $100000 \mathrm{~K}$ (e.g., Dere et al., 1989; Chae et al., 1998b; Winebarger et al., 1999, 2002b,a). However, there are indications that such EUV explosive events are not directly relevant in heating the corona, are characteristic of structures not obviously connected with the upper corona, and have a chromospheric origin (Teriaca et al., 2002). Moderate outflows at about 1 MK or more have been found from combined SoHO/SUMER and Hinode/EIS observations of the quiet Sun around disk center (Dadashi et al., 2011).

Doppler shifts have been extensively studied as a function of temperature across the transition region. Figure 10 shows a summary of the results updated to 2011.

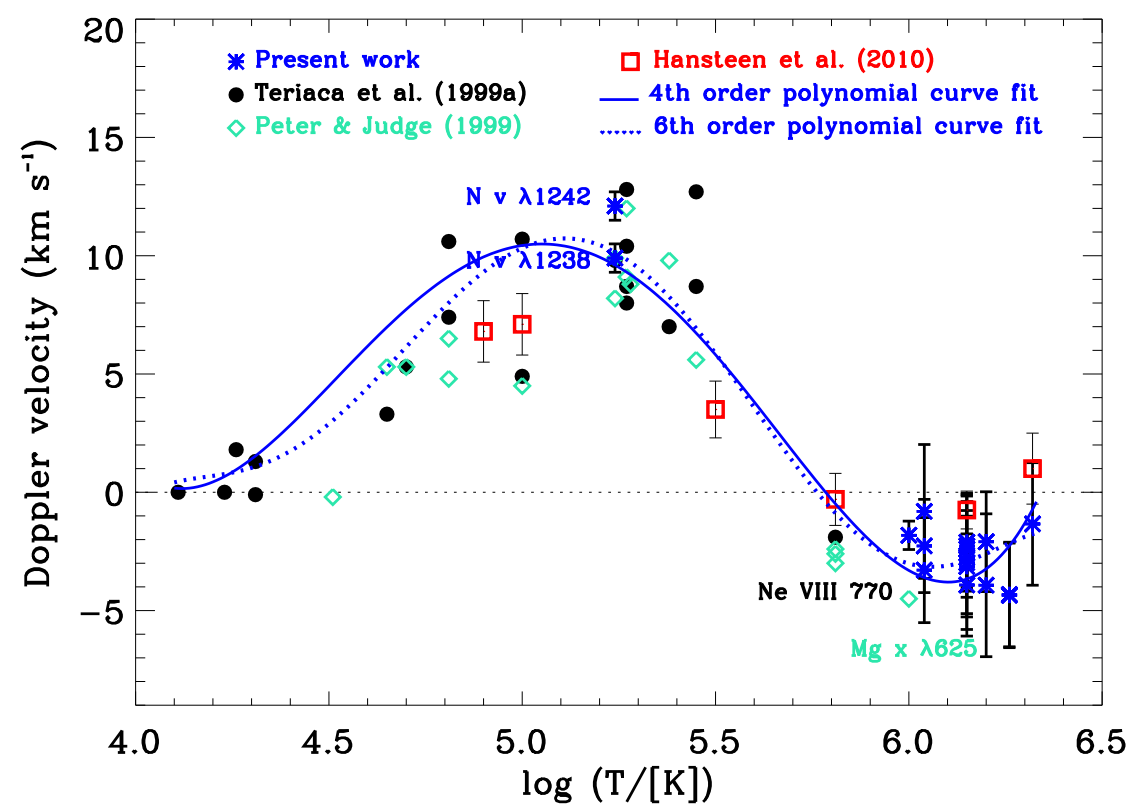

Figure 10: Average Doppler shift in the quiet Sun at disk center of various TR and coronal ions measured from SUMER and EIS spectra. Positive/negative values indicate red- (downflows) / blue- (upflows) shifts. Solid and dashed lines respectively represent polynomial fittings. Image reproduced with permission from Dadashi et al. (2011), copyright by ESO.

Redshifts increasing up to $\sim 10 \mathrm{~km} \mathrm{~s}^{-1}$ at $\sim 2 \times 10^{5} \mathrm{~K}$ and then decreasing with increasing temperature have been found from SUMER and EIS spectra in the quiet Sun and explained by the dominance of emission from plasma flowing downward from the upper hot region to the lower cool region along flux tubes with varying cross section (a factor about 30, Chae et al., 1998c, see also Section 3.1). The redshift peak increases to $15 \mathrm{~km} \mathrm{~s}^{-1}$ in an active region at temperature of $10^{5} \mathrm{~K}$, while the redshifts were found to turn into blueshifts at temperatures above $5 \times 10^{5} \mathrm{~K}$ (Teriaca et al., 1999). The trend from redshift to blueshift also applies to active region moss, but the transition from red to blue appears to occur at a higher temperature in the moss $(\sim 1 \mathrm{MK}$ versus 0.5 MK in the quiet Sun) (Dadashi et al., 2012; Tripathi et al., 2012b).

Regarding the spatial localization of the flows, a complex scenario of Doppler flows was found in active region loops observed by Hinode EIS (Del Zanna, 2008, Figure 11). Persistent redshifts, stronger in cooler lines (about $5-10 \mathrm{~km} \mathrm{~s}^{-1}$ in Fe XII and $20-30 \mathrm{~km} \mathrm{~s}^{-1}$ in Fe VIII), were confirmed in most loop structures. Persistent blueshifts, stronger in the hotter lines (typically $5-20 \mathrm{~km} \mathrm{~s}^{-1}$ in Fe XII and $10-30 \mathrm{~km} \mathrm{~s}^{-1}$ in FeXV), were present in areas of weak emission, in a sharp boundary 
between the low-lying "hot" 3 MK loops and the higher "warm" 1 MK loops.

An active region was comprised of red-shifted emissions (downflows) in the core and blueshifted emissions (upflows) at the boundary (Tripathi et al., 2009). No strong flows were found in an active region core in the Hinode/EIS Fe XII $195 \AA$ line (Brooks and Warren, 2009). In the core of moss regions SUMER and EIS data show some blueshifts of a few $\mathrm{km} \mathrm{s}^{-1}$ at low coronal temperatures, decreasing at higher temperatures (Dadashi et al., 2012). Specific studies of coronal loops in an active region show mostly blue-shifted emission at coronal temperatures, with speed of about $20 \mathrm{~km} \mathrm{~s}^{-1}$ at the footpoints (Tripathi et al., 2012a).


Figure 11: Monochromatic (negative) images and dopplergrams $\mathrm{km} \mathrm{s}^{-1}$ ) of an active region (NOAA 10926) observed with Hinode/EIS in Fe viII, Fe xII, Fexv lines. Courtesy of G. Del Zanna.

Non-thermal velocities in the transition region and corona of the quiet Sun were measured from the widths of SoHO/SUMER UV lines (Chae et al., 1998a). They were found to increase with temperature from values smaller than $10 \mathrm{~km} \mathrm{~s}^{-1}$ at temperatures $<2 \times 10^{4} \mathrm{~K}$, to a peak value of $30 \mathrm{~km} \mathrm{~s}^{-1}$ around $3 \times 10^{5}$, and then to increase with temperature, to about $20 \mathrm{~km} \mathrm{~s}^{-1}$ at coronal temperatures. Since the motions are small-scale and isotropic they were interpreted in terms of MHD turbulence. Non-thermal velocities were studied also in solar active regions on Hinode/EIS spectra (Doschek et al., 2007). The largest widths seem to be located more in relatively faint zones, some of which also show Doppler outflows.

Coronal plasma motions near footpoints of active region loops showed a strong correlation between Doppler velocity and non-thermal velocity (Hara et al., 2008). Significant deviations from a single Gaussian profile were found in the blue wing of the line profiles for the upflows. These may suggest that there are unresolved high-speed upflows.

EUV spectra of coronal loops above active regions show also clear evidence of stronger dynamical activity. In the Ov $629 \AA$ line, formed at $240000 \mathrm{~K}$, line-of-sight velocities greater than $50 \mathrm{~km} \mathrm{~s}^{-1}$ have been measured with the shift extending over a large fraction of a loop (Brekke et al., 1997). Active region loops appear to be extremely time variable and dynamic at transition region temperatures, with large Doppler shifts (Brekke, 1999). The birth, evolution and cooling of one of such transient cool loops was directly observed with the SoHO/CDS, and a blue-shifted upflow was measured all along the loop, probably a one-direction siphon flow (Di Giorgio et al., 2003, see also Section 3.5). Line-of-sight flows of up to $40 \mathrm{~km} \mathrm{~s}^{-1}$ were measured along warm and apparently static active region loops in co-aligned TRACE and the SoHO/SUMER observations (Winebarger et al., 2002c). Apparent motions were also detected in other TRACE images (Winebarger et al., 2001). Strong localized outflows $\left(\sim 50 \mathrm{~km} \mathrm{~s}^{-1}\right)$ in a widespread downflow region were clearly visible in Doppler-shifts maps obtained with EIS (Doschek et al., 2008). The outflows might be tracers 
of long loops and/or open magnetic fields. High-speed outflows at about 1 MK or more have been found in microflares (Chen and Ding, 2010), and transient ones at the boundary of active region cores on the timescale of minutes in time sequences of EIS spectra (Ugarte-Urra and Warren, 2011). Studies on the temperature structure and chemical composition of the plasma producing the faint blue wings at about $100 \mathrm{~km} \mathrm{~s}^{-1}$ have shown a peaks at coronal temperatures above $1 \mathrm{MK}$ and coronal FIP bias values (Brooks and Warren, 2012). Cool plasma flowing in multi-threaded coronal loops were detected in high resolution Hinode SOT observations with speeds in the range $74-123 \mathrm{~km} \mathrm{~s}^{-1}$ (Ofman and Wang, 2008). In addition to flows, the loops exhibited transverse oscillations. Even stronger upflows, more typical of flare chromospheric evaporation (e.g., Antonucci et al., 1982), have also been detected during some small transient brightenings in the X-ray band (Hinode/XRT), with speeds up to $500 \mathrm{~km} \mathrm{~s}^{-1}$ (Nitta et al., 2012).

\subsubsection{Chromospheric flows}

It has been proposed that an important role in the dynamics and heating of plasma inside coronal loops might be played by upcoming chromospheric flows. Although the discovery is not new (Athay and Holzer, 1982), recently much attention has been devoted to these flows, and in particular to a specific class of finger-like ejections with speeds between 50 and $150 \mathrm{~km} \mathrm{~s}^{-1}$ and lasting a couple of minutes or less, the so-called type II spicules (De Pontieu et al., 2007a). There is evidence for a spatial and temporal correlation between spicules and faint upflows detected in the EUV band suggesting the possibility that the flows are heated to coronal temperatures when they are injected in coronal loops. The upflows were detected as a blue-shifted excess (wing asymmetry) in an EUV line (Fe XIV). The implication is that spicules may provide a substantial contribution to coronal heating, thus shifting the source of coronal heating down in the chromosphere (De Pontieu et al., 2009) and proposing a new challenge for coronal heating theory. The evidence is debated. Wavelike propagations in coronal EUV images have been also re-interpreted as evidence for repetitive upflows (Tian et al., 2011; Kamio et al., 2011). However, 3D MHD modeling shows that waves and flows may results from the same impulsive events (Ofman et al., 2012), while flows may be important very low in the corona, the propagating disturbances are dominated by waves at higher altitudes in active region loop (Wang et al., 2013). Coronal counterparts have been directly identified in EUV images (De Pontieu et al., 2011) and also a correspondence between upflows and downflows, the latter at moderate speed $\left(\sim 10 \mathrm{~km} \mathrm{~s}^{-1}\right)$ and in the cool passbands (McIntosh et al., 2012). These are to be compared with the usual evidence of redshifts in the transition region. In other analyses high speed spicules were not found to have coronal counterparts (Madjarska et al., 2011) and to be a separate population from lower speed spicules (Zhang et al., 2012). It has been estimated that only a small fraction of coronal plasma can be supplied by chromospheric upflows (Klimchuk, 2012).

\subsubsection{Waves observations}

Although this review focusses more on the plasma confined in loops, recently considerable efforts have been devoted to models that point to the importance of magnetohydrodynamic waves. The question is again the contribution of the dissipation of wave energy to the heating of the corona, that is not easy to evaluate (Klimchuk, 2006). The wave propagation is often connected to and needs to be distinguished from the presence of flows. Therefore, we dedicate attention to recent evidences regarding coronal waves and oscillations that involve loops.

Evidence for photospheric Alfvén waves was obtained from magnetic and velocity fluctuations in regions of strong magnetic field (Ulrich, 1996) and from granular motions in the quiet Sun (Muller et al., 1994) with fluxes of the order of $10^{7} \mathrm{erg} \mathrm{cm}^{-2} \mathrm{~s}^{-1}$, which might contribute to heating if transmitted efficiently to the corona. 
In SoHO/EIT high-cadence $304 \AA$ images, analyzed systematic intensity variations along an offlimb half loop structure were observed to propagate from the top toward the footpoints (De Groof et al., 2004). These intensity variations are more probably be due to flowing/falling plasma blobs than to slow magneto-acoustic waves (Section 4.4). This evidence has been addressed also by modeling studies (see Section 4.3).

Widespread evidence for outward propagation of Alfvén waves is reported from ground optical polarimetric observations (Tomczyk et al., 2007), and non-thermal broadening has been shown to correlate (McIntosh and De Pontieu, 2012) with swaying motions detected in the corona from SDO/AIA data (speed of $\sim 20 \mathrm{~km} \mathrm{~s}^{-1}$ and periods of few minutes) (McIntosh et al., 2011). TRACE EUV observations provided the first evidence of resolved transverse waves in coronal loops (Aschwanden et al., 1999a; Nakariakov et al., 1999; Nakariakov and Ofman, 2001). Undamped, or even growing waves were observed by SDO/AIA (Wang et al., 2012; Nisticò et al., 2013). Transverse waves were detected also in high resolution observations with the $\mathrm{HiC}$ in thin loops $(\geq 100 \mathrm{~km})$ at low speed amplitude (Morton and McLaughlin, 2013). The contribution of this class of waves is estimated, discussed, and debated although there is still no clear convergence. Other oscillations were observed to propagate along coronal loops at more than $100 \mathrm{~km} \mathrm{~s}^{-1}$ with periods of a few minutes and interpreted as slow magnetosonic waves (De Moortel et al., 2000), but also as due to faint upflows (De Pontieu and McIntosh, 2010). We mention that also much faster ( $>1000 \mathrm{~km} \mathrm{~s}^{-1}$ ) propagating oscillations were detected in SDO/AIA observations during eruptive events and interpreted as fast magnetosonic waves (e.g., Liu et al., 2011; Ofman et al., 2011). 


\section{Loop Physics and Modeling}

\subsection{Basics}

The basics of loop plasma physics are well established since the 1970s (e.g., Priest, 1978). In typical coronal conditions, i.e., ratio of thermal and magnetic pressure $\beta \ll 1$, temperature of a few MK, density of $10^{8}-10^{10} \mathrm{~cm}^{-3}$, the plasma confined in coronal loops can be assumed as a compressible fluid moving and transporting energy only along the magnetic field lines, i.e., along the loop itself (e.g., Rosner et al., 1978; Vesecky et al., 1979). In this configuration, the magnetic field has only the role of confining the plasma. It is also customary to assume constant loop cross-section (see Section 3.2.1). In these conditions, and neglecting gradients across the direction of the field, effects of curvature, non uniform loop shape, magnetic twisting, currents and transverse waves, the plasma evolution can be described by means of the one-dimensional hydrodynamic equations for a compressible fluid, using only the coordinate along the loop (Figure 12).



Figure 12: The plasma confined in a loop can be described with one-dimensional hydrodynamic modeling, with a single coordinate $(s)$ along the loop. Image: TRACE, 6 November 1999, 2 UT.

The time-dependent equations of mass, momentum, and energy conservation typically include the effects of the gravity component along the loop, the radiative losses from an optically thin plasma, the plasma thermal conduction, an external heating input, the plasma compressional viscosity:

$$
\begin{gathered}
\frac{d n}{d t}=-n \frac{\partial v}{\partial s}, \\
n m_{\mathrm{H}} \frac{d v}{d t}=-\frac{\partial p}{\partial s}+n m_{\mathrm{H}} g+\frac{\partial}{\partial s}\left(\mu \frac{\partial v}{\partial s}\right), \\
\frac{d \epsilon}{d t}+(p+\epsilon) \frac{\partial v}{\partial s}=H-n^{2} \beta_{i} P(T)+\mu\left(\frac{\partial v}{\partial s}\right)^{2}+F_{c},
\end{gathered}
$$


with $p$ and $\epsilon$ defined by:

$$
p=\left(1+\beta_{i}\right) n k_{\mathrm{B}} T, \quad \epsilon=\frac{3}{2} p+n \beta_{i} \chi,
$$

and the conductive flux:

$$
F_{c}=\frac{\partial}{\partial s}\left(\kappa T^{5 / 2} \frac{\partial T}{\partial s}\right)
$$

where $n$ is the hydrogen number density, $s$ the spatial coordinate along the loop, $v$ the plasma velocity, $m_{\mathrm{H}}$ the mass of hydrogen atom, $\mu$ the effective plasma viscosity, $P(T)$ the radiative losses function per unit emission measure (e.g., Raymond et al., 1976), $\beta_{i}$ the fractional ionization, i.e., $n_{\mathrm{e}} / n_{\mathrm{H}}, F_{c}$ the conductive flux, $\kappa$ the thermal conductivity (Spitzer, 1962), $k_{\mathrm{B}}$ the Boltzmann constant, and $\chi$ the hydrogen ionization potential. $H(s, t)$ is a function of both space and time that describes the heat input in the loop.

These equations can be solved numerically and several specific codes have been used extensively to investigate the physics of coronal loops and of X-ray flares (e.g., Nagai, 1980; Peres et al., 1982; Doschek et al., 1982; Nagai and Emslie, 1984; Fisher et al., 1985a,a,a; MacNeice, 1986; Gan et al., 1991; Hansteen, 1993; Betta et al., 1997; Antiochos et al., 1999; Ofman and Wang, 2002; Müller et al., 2003; Bradshaw and Mason, 2003; Sigalotti and Mendoza-Briceño, 2003; Bradshaw and Cargill, 2006).

The concept of numerical loop modeling is to use simulations, first of all, to get insight into the physics of coronal loops, i.e., the reaction of confined plasma to external drivers, to describe plasma evolution, and to derive predictions to compare with observations. One major target of modeling is of course to discriminate between concurrent hypotheses, for instance, regarding the heating mechanisms, and to constrain the related parameters.

The models require to be provided with initial loop conditions and boundary conditions. It has been shown that time-dependent loop models must include a relatively thick, cool, and dense chromosphere and the transition region for a correct description of the mass transfer driven by transient heating (e.g., Bradshaw and Cargill, 2013) and to maintain the necessary numerical stability (Antiochos, 1979; Hood and Priest, 1980; Peres et al., 1982). The main role of the chromosphere is only that of a mass reservoir and, therefore, in several codes, it is treated as simply as possible, e.g., an isothermal inactive layer that neither emits, nor conducts heat. In other cases, a more accurate description is chosen, e.g., including a detailed chromospheric model (e.g., Vernazza et al., 1981), maintaining a simplified radiative emission and a detailed energy balance with an ad hoc heat input (Peres et al., 1982; Reale et al., 2000a). Overall, a typical loop initial condition is a hydrostatic atmosphere with a temperature distribution from $\sim 10^{4} \mathrm{~K}$ to $>10^{6} \mathrm{~K}$, basically dictated by a thermal conduction profile (Figure 14). The lower boundary of the computational domain is typically not involved in the evolution of the loop plasma. Many loop models assume mirror symmetry with respect to the apex and, therefore, describe only half of the loop. The upper boundary conditions are those of symmetry at the loop apex.

The models also require to define an input heating function (see Section 4.4), specifying its time-dependence, for instance it can be steady, slowly, or impulsively changing, and its position in space. The output typically consists of distributions of temperature, density, and velocity along the loop evolving with time. From simulation results, some modelers derive observables, i.e., the plasma emission, which can be compared directly to data collected with the telescopes. The model results are, in this case, to be folded with the instrumental response. This forward-modeling allows to obtain constraints on model parameters and, therefore, quantitative information about the questions to be solved, e.g., the heating rate and location (e.g., Reale et al., 2000a).

Loop codes are typically based on finite difference numerical methods. Although they are onedimensional and, therefore, typically less demanding than other multi-dimensional codes that study systems with more complex geometry, and although they do not include the explicit description of the magnetic field, as full MHD codes, loop codes require some special care. One of the main 
difficulties consists in the appropriate resolution of the steep transition region $(1-100 \mathrm{~km}$ thick) between the chromosphere and the corona, which can easily drift up and down depending on the dynamics of the event to be simulated. The temperature gradient there is very large due to the local balance between the steep temperature dependence of the thermal conduction and the peak of the radiative losses function (Serio et al., 1981). The density is steep as well so to maintain the pressure balance. The transition region can become very narrow during flares. An insufficient resolution of the transition region can lead to inaccurate description of the loop plasma dynamics, e.g., chromospheric evaporation (Bradshaw and Cargill, 2013, see Section 4.1.2). Also a fine temporal resolution is extremely important, because the highly efficient thermal conduction in a hot magnetized plasma can lead to a very small time step and make execution times not so small even nowadays. Some deviations can be possible because of non-local thermal conduction that may lengthen considerably the conduction cooling times and may enhance the chances of observing hot nanoflare-heated plasma (West et al., 2008).

In recent years, time-dependent loop modeling has been revived in the light of the observations with SoHO, TRACE, and SDO for the investigation of the loop dynamics and heating. The upgrade driven by the higher quality of the data has consisted in the introduction of more detailed mechanisms for the heating input, for the momentum deposition, or others, e.g., the time-dependent ionization and the saturated thermal conduction (Bradshaw and Cargill, 2006; Reale and Orlando, 2008). Some codes have been upgraded to include adaptive mesh refinement for better resolution in regions of high gradients, such as in the transition region, or during impulsive events (e.g., Betta et al., 1997). Another form of improvement has been the description of loops as collections of thin strands. Each strand is a self-standing, isolated and independent atmosphere, to be treated exactly as a single loop. This approach has been adopted both to describe loops as static (Reale and Peres, 2000) (Figure 13) and as impulsively heated by nanoflares (Warren et al., 2002). On the same line, collections of loop models have been applied to describe entire active regions (Warren and Winebarger, 2006).

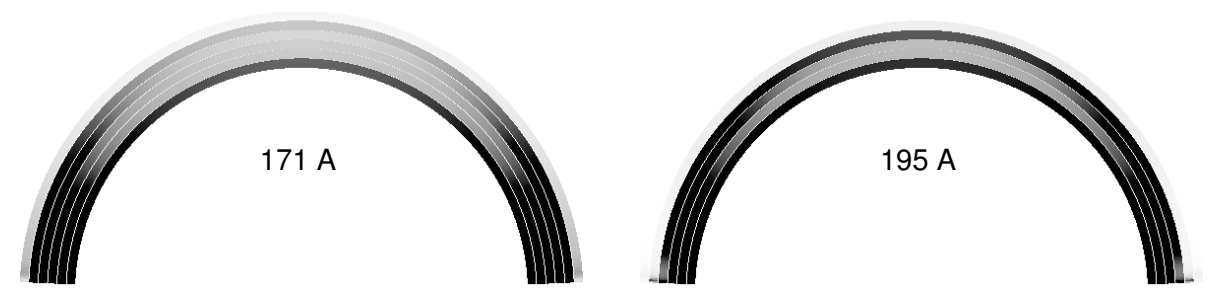

0

$2.5 \cdot 10^{-10}$ $5.0 \cdot 10^{-10}$

Figure 13: Emission in two TRACE filterbands predicted by a model of loop made by several thin strands. Image reproduced with permission from Reale and Peres (2000), copyright by AAS.

One limitation of current $1 \mathrm{D}$ loop models is that they are unable to treat conveniently the tapering expected going down from the corona to the chromosphere (or expansion upwards) through the transition region. This effect can be neglected in many circumstances, but it is becoming increasingly important with the finer and finer level of diagnostics allowed by upcoming observational data. For instance, the presence of tapering changes considerably the predicted distribution 
of emission measure in the low temperature region (Section 4.1.1).

Possible deviations from pure 1D evolution might be driven by intense oscillations or kinks, as described in Ofman (2009). The effect of the three-dimensional loop structure should then be taken into account to describe the interaction with excited MHD waves (McLaughlin and Ofman, 2008; Pascoe et al., 2009; Selwa and Ofman, 2009).

However, the real power of $1 \mathrm{D}$ loop models, that makes them still on the edge, is that they fully exploit the property of the confined plasma to evolve as a fluid and practically independent of the magnetic field, and that they can include the coronal part, the transition region, and the photospheric footpoint in a single model with thermal conduction. In this framework, we may even simulate a multi-thread structure only by collecting many single loop models together, still with no need to include the description and interaction with the magnetic field (Guarrasi et al., 2010). We should, however, be aware that the magnetic confinement of the loop material is not as strong and the thermal conduction is not as anisotropic below the coronal part of the loop as it is in the corona.

An efficient approach to loop modeling is to describe the temporal evolution of average loop quantities (temperature, pressure, and density), i.e., a "0-D" model (Klimchuk et al., 2008; Cargill et al., 2012a,b). This model is useful for the description of loops as collections of myriads of independent strands with a statistical distribution of heating events.

Alternative approaches to single or multiple loop modeling have been developed more recently, thanks also to the increasing availability of high performance computing systems and resources. Global "ab initio" approaches have been developed (Gudiksen and Nordlund, 2005; Hansteen et al., 2007; see also Yokoyama and Shibata, 2001 for the case of a flare model) to model - with full MHD - boxes of the solar corona that span the entire solar atmosphere from the upper convection zone to the lower corona. These models include non-grey, non-LTE (local thermodynamic equilibrium) radiative transport in the photosphere and chromosphere, optically thin radiative losses, as well as magnetic field-aligned heat conduction in the transition region and corona. Although such models still cannot resolve well fine structures, such as current sheets and the transition region, they certainly represent the first important step toward fully self-consistent modeling of the magnetized corona. Large-scale MHD modeling has been used to explain the appearance of constant crosssection in EUV observations as due to temperature variations across the loop (Peter and Bingert, 2012). Another global model of the solar corona includes also information from photospheric magnetic field data (Sokolov et al., 2013).

\subsubsection{Monolithic (static) loops: scaling laws}

The Skylab mission remarked, and later missions confirmed (Figure 9), that many X-ray emitting coronal loops persist mostly unchanged for a time considerably longer than their cooling times by radiation and/or thermal conduction (Rosner et al., 1978, and references therein). This means that, for most of their lives, they can be well described as systems at equilibrium and has been the starting point for several early theoretical studies (Landini and Monsignori Fossi, 1975; Gabriel, 1976; Jordan, 1976; Vesecky et al., 1979; Jordan, 1980). Rosner et al. (1978) devised a model of coronal loops in hydrostatic equilibrium with several realistic simplifying assumptions: symmetry with respect to the apex, constant cross section (see Section 3.2.1), length much shorter than the pressure scale height, heat deposited uniformly along the loop, low thermal flux at the base of the transition region, i.e., the lower boundary of the model. Under these conditions, the pressure is uniform all along the loop, which is then described only by the energy balance between the heat input and the two main losses mentioned above. From the integration of the equation of energy conservation, one obtains the well-known scaling laws:

$$
T_{0,6}=1.4\left(p L_{9}\right)^{1 / 3}
$$


and

$$
H_{-3}=3 p^{7 / 6} L_{9}^{-5 / 6},
$$

where $T_{0,6}, L_{9}$ and $H_{-3}$ are the loop maximum temperature $T_{0}$, length $L$ and heating rate per unit volume $H$, measured in units of $10^{6} \mathrm{~K}(\mathrm{MK}), 10^{9} \mathrm{~cm}$ and $10^{-3} \mathrm{erg} \mathrm{cm}^{-3} \mathrm{~s}^{-1}$ respectively. These scaling laws were found in agreement with Skylab data within a factor 2.

Analogous models were developed in the same framework (Landini and Monsignori Fossi, 1975) and equivalent scaling laws were found independently by Craig et al. (1978) and more general ones by Hood and Priest (1979a). They have been derived with a more general formalism by Bray et al. (1991). Although scaling laws could explain several observed properties, some features such as the emission measure in UV lines and the cool loops above sunspots could not be reproduced, and, although the laws have been questioned a number of times (e.g., Kano and Tsuneta, 1995) in front of the acquisition of new data, such as those by Yohkoh and TRACE, they anyhow provide a basic physical reference frame to interpret any loop feature. For instance, they provide reference equilibrium values even for studies of transient coronal events, they have allowed to constrain that many loop structures observed with TRACE are overdense (e.g., Lenz et al., 1999; Winebarger et al., 2003a, Section 4.1.2) and, as such, these loops must be cooling from hotter status (Winebarger and Warren, 2005, see Section 3.3.3), and so on. They also are useful for density estimates when closed with the equation of state, and for coronal energy budget when integrated on relevant volumes and times.

Scaling laws have been extended to loops higher than the pressure scale height (Serio et al., 1981), to different heating functions (Martens, 2010), and limited by the finding that very long loops become unstable (Wragg and Priest, 1981). According to Antiochos and Noci (1986), the cool loops belong to a different family and are low-lying, and may eventually explain an evidence of excess of emission measure at low temperature.

The numerical solution of the complete set of hydrostatic equations allowed to obtain detailed profiles of the physical quantities along the loop, including the steep transition region. Figure 14 shows two examples of solution for different values of heating uniformly distributed along the loop.

Hydrostatic weighting has an effect on the loop visibility and on the vertical temperature structure of the solar corona (Reale, 1999; Aschwanden and Nitta, 2000). From the comparison of SoHO-CDS observations of active region loops with a static, isobaric loop model (Landini and Landi, 2002), a classical model was not able to reproduce the observations, but ad hoc assumptions are necessary (Brković et al., 2002; Landi and Landini, 2004). Loop static models were found to overestimate the footpoint emission by orders of magnitude and non-uniformity in the loop cross section, more specifically a significant decrease of the cross section near the footpoints, was proposed as the most likely solution to the discrepancy (Landi and Feldman, 2004, Section 4.1). On the same line, loop models with steady uniform heating were compared to X-ray loops and EUV moss in an active region core (Winebarger et al., 2008). A filling factor of $8 \%$ and loops that expand with height provided the best agreement with the intensity in two X-ray filters, though maintaining still some discrepancies with observations. A simple electrodynamic model was useful to evaluate the connection of electric currents and heating to the loop cross-section in a solar active region (Gontikakis et al., 2008).

The strength of scaling laws is certainly their simplicity and their easy and general application, even in the wider realm of stellar coronae. However, increasing evidence of dynamically heated, fine structured loops is indicating the need for improvements.

\subsubsection{Structured (dynamic) loops}

In the scenario of loops consisting of bundles of thin strands, each strand behaves as an independent atmosphere and can be described as an isolated loop itself. If the strands are numerous and heated independently, a loop can be globally maintained steady with a sequence of short heat pulses, each 

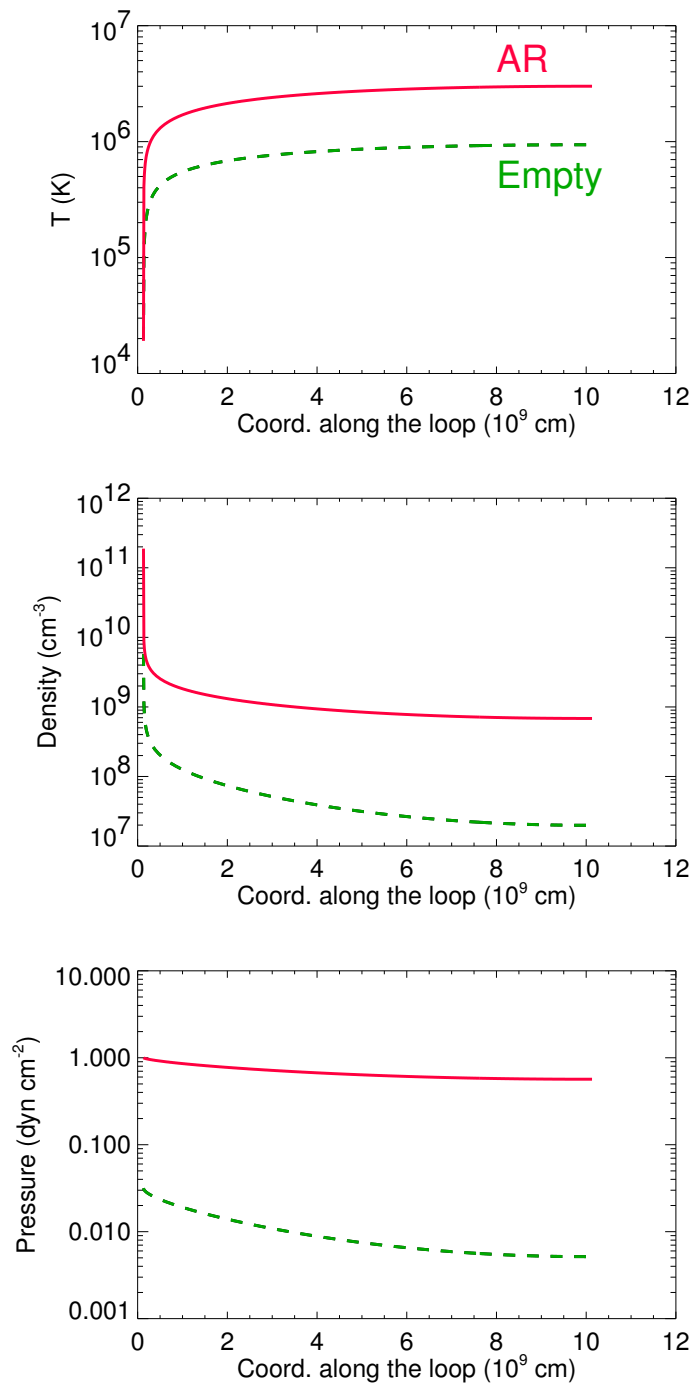

Figure 14: Distributions of temperature, density, and pressure along a hydrostatic loop computed from the model of Serio et al. (1981) for a high pressure loop (AR) and a low pressure one (Empty) with heating uniformly distributed along the loop. 
igniting a single or a few strands (nanoflares). In this case, although the loop remains steady on average for a long time, each strand has a continuously dynamic evolution. The evolution of a loop structure under the effect of an impulsive heating is well-known and studied from observations and from modeling (e.g., Nagai, 1980; Peres et al., 1982; Cheng et al., 1983; Nagai and Emslie, 1984; Fisher et al., 1985a,b,c; MacNeice, 1986; Betta et al., 2001), since it resembles the evolution of single coronal flaring loops. It is worth mentioning here that there have been attempts to model even flaring loops as consisting of several flaring strands (Hori et al., 1997, 1998; Reeves and Warren, 2002; Warren, 2006; Reale et al., 2012).

The evolution of single coronal loops or single loop strands subject to impulsive heating was summarized in the context of the diagnostics of stellar flares (Reale, 2007). A heat pulse injected in an inactive tenuous strand makes chromospheric plasma expand in the coronal section of the strand, and become hot and dense, X-ray bright, coronal plasma. After the end of the heat pulse, the plasma begins to cool slowly. In general, the plasma cooling is governed by the thermal conduction to the cool chromosphere and by radiation from optically thin conditions. In the following, we outline the evolution of the confined heated plasma into four phases, according to Reale (2007). Figure 15 tracks this evolution, which maps on the path drawn in the density-temperature diagram of Figure 16 (see also Jakimiec et al., 1992).

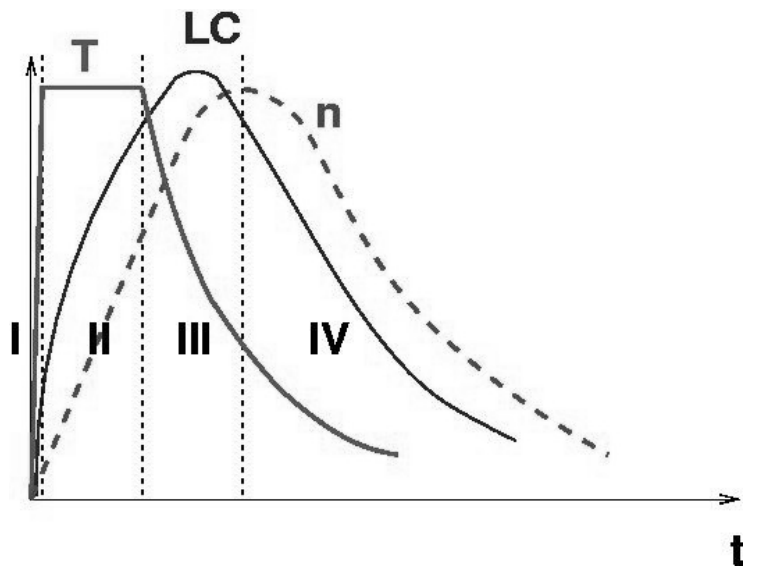

Figure 15: Scheme of the evolution of temperature ( $T$, thick solid line), X-ray emission, i.e., the light curve (LC, thinner solid line) and density (n, dashed line) in a loop strand ignited by a heat pulse. The strand evolution is divided into four phases (I, II, III, IV, see text for further details). Image reproduced with permission from Reale (2007), copyright by ESO.

Phase I: From the start of the heat pulse to the temperature peak (heating). If the heat pulse is triggered in the coronal part of the loop, the heat is efficiently conducted down to the much cooler and denser chromosphere. The temperature rapidly increases in the whole loop, with a timescale given by the conduction time in a low density plasma (see below). This evolution changes only slightly if the heat pulse is deposited near the loop footpoints: the conduction front then propagates mainly upwards and on timescales not very different from the evaporation timescales, also because the heat conduction saturates (e.g., Klimchuk, 2006; Reale and Orlando, 2008). In this case the distinction from Phase II is not clearly marked.

Phase II: From the temperature peak to the end of the heat pulse (evaporation). The temperature settles to the maximum value $\left(T_{0}\right)$. The chromospheric plasma is strongly heated, expands upwards, and fills the loop with much denser plasma. This occurs both if the heating is conducted from the highest parts of the corona and if it released directly near the 
loop footpoints. The evaporation is explosive at first, with a timescale given by the isothermal sound crossing time $(s)$, since the temperature is approximately uniform in the highly conductive corona:

$$
\tau_{s d}=\frac{L}{\sqrt{2 k_{B} T_{0} / m}} \approx 80 \frac{L_{9}}{\sqrt{T_{0,6}}}
$$

where $m$ is the average particle mass. After the evaporation front has reached the loop apex, the loop continues to fill more gently. The timescale during this more gradual evaporation is dictated by the time taken by the cooling rate to balance the heat input rate.

Phase III: From the end of the heat pulse to the density peak (conductive cooling). When the heat pulse stops, the plasma immediately starts to cool due to the efficient thermal conduction (e.g., Cargill and Klimchuk, 2004), with a timescale (s):

$$
\tau_{c}=\frac{3 n_{c} k_{B} T_{0} L^{2}}{2 / 7 \kappa T_{0}^{7 / 2}}=\frac{10.5 n_{c} k_{B} L^{2}}{\kappa T_{0}^{5 / 2}} \approx 1500 \frac{n_{9} L_{9}^{2}}{T_{6}^{5 / 2}}
$$

where $n_{c}\left(n_{c, 9}\right)$ is the particle density $\left(10^{9} \mathrm{~cm}^{-3}\right)$ at the end of the heat pulse, the thermal conductivity is $\kappa=9 \times 10^{-7}$ (c.g.s. units). Since the plasma is dense, we expect no saturation effects in this phase.

The heat stop time can be generally traced as the time at which the temperature begins to decrease significantly and monotonically. While the conduction cooling dominates, the plasma evaporation is still going on and the density increasing. The efficiency of radiation cooling increases as well, while the efficiency of conduction cooling decreases with the temperature.

Phase IV: From the density peak afterwards (Radiative cooling). As soon as the radiation cooling time becomes equal to the conduction cooling time (Cargill and Klimchuk, 2004), the density reaches its maximum, and the loop depletion starts, slowly at first and then progressively faster. The pressure begins to decrease inside the loop, and is no longer able to sustain the plasma. The radiation becomes the dominant cooling mechanism, with the following timescale $(s)$ :

$$
\tau_{r}=\frac{3 k_{B} T_{M}}{n_{M} P(T)}=\frac{3 k_{B} T_{M}}{b T_{M}^{\alpha} n_{M}} \approx 3000 \frac{T_{M, 6}^{3 / 2}}{n_{M, 9}},
$$

where $T_{M}\left(T_{M, 6}\right)$ is the temperature at the time of the density maximum $\left(10^{6} \mathrm{~K}\right), n_{M}$ $\left(n_{M, 9}\right)$ the maximum density $\left(10^{9} \mathrm{~cm}^{-3}\right)$, and $P(T)$ the plasma emissivity per unit emission measure, expressed as:

$$
P(T)=b T^{\alpha},
$$

with $b=1.5 \times 10^{-19}$ and $\alpha=-1 / 2$. The density and the temperature both decrease monotonically.

The presence of significant residual heating could make the decay slower. In single loops, this can be diagnosed from the analysis of the slope of the decay path in the density-temperature diagram (Sylwester et al., 1993; Reale et al., 1997a). The free decay has a slope between 1.5 and 2 in a $\log$ density vs log temperature diagram; heated decay path is flatter down to a slope $\sim 0.5$. In non-flaring loops, the effect of residual heating can be mimicked by the effect of a strong gravity component, as in long loops perpendicular to the solar surface. The dependence of the decay slope on the pressure scale height has been first studied in Reale et al. (1993) and, more recently, in terms of enthalpy flux by Bradshaw and Cargill (2010).

As clear from Figure 16 the path in this phase is totally below, or at most approaches, the QSS curve. This means that for a given temperature value the plasma density is higher than 
that expected for an equilibrium loop at that temperature, i.e., the plasma is "overdense". Evidence of such overdensity (Section 3.3.3) has been taken as an important indication of steadily pulse-heated loops.

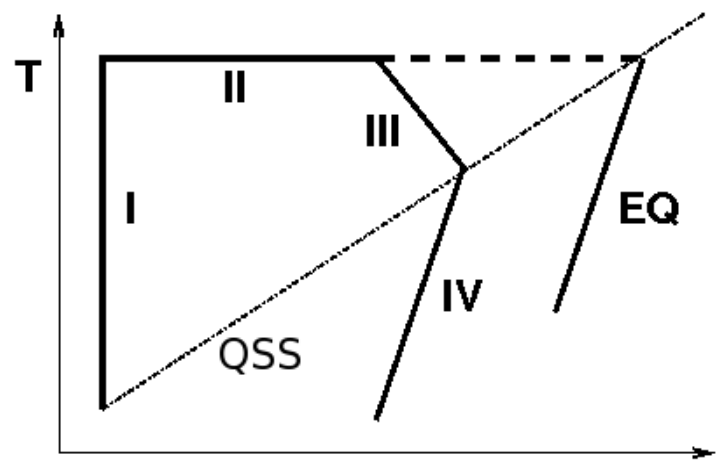

Figure 16: Scheme of the evolution of pulse-heated loop plasma of Figure 15 in a density-temperature diagram (solid line). The four phases are labeled. The locus of the equilibrium loops is shown (dasheddotted line, marked with QSS), as well as the evolution path with an extremely long heat pulse (dashed line) and the corresponding decay path (marked with EQ). Image adapted from Reale (2007), copyright by ESO.

This is the evolution of a loop strand ignited by a transient heat pulse. Important properties of the heated plasma can be obtained from the analysis of the evolution after the heating stops, i.e., when the plasma cools down.

Serio et al. (1991) derived a global thermodynamic timescale for the pure cooling of heated plasma confined in single coronal loops, which has been later refined to be (s) (Reale, 2007):

$$
\tau_{s}=4.8 \times 10^{-4} \frac{L}{\sqrt{T_{0}}}=500 \frac{L_{9}}{\sqrt{T_{0,6}}} .
$$

This decay time was obtained assuming that the decay starts from equilibrium conditions, i.e., departing from the locus of the equilibrium loops with a given length (hereafter QSS line, Jakimiec et al., 1992) in Figure 16. It is, therefore, valid as long as there is no considerable contribution from the plasma draining to the energy balance. The link between the assumption of equilibrium and the plasma evolution is shown in Figure 16: if the heat pulse lasts long enough, Phase II extends to the right, and the heated loop asymptotically reaches equilibrium conditions, i.e., the horizontal line approaches the QSS line. If the decay starts from equilibrium conditions, Phase III is no longer present, and Phase II links directly to Phase IV. Therefore, there is no delay between the beginning of the temperature decay and the beginning of the density decay: the temperature and the density start to decrease simultaneously. Also, the decay will be dominated by radiative cooling, except at the very beginning (Serio et al., 1991).

The presence of Phase III implies a delay between the temperature peak and the density peak. This delay is often observed both in solar flares (e.g., Sylwester et al., 1993) and in stellar flares (e.g., van den Oord et al., 1988; van den Oord and Mewe, 1989; Favata et al., 2000; Maggio et al., 2000; Stelzer et al., 2002). The presence of this delay, whenever observed, is a signature of a relatively short heat pulse, or, in other words, of a decay starting from non-equilibrium conditions.

According to Reale (2007), the time taken by the loop to reach equilibrium conditions under the action of a constant heating is much longer than the sound crossing time [Eq. (11)], which rules the very initial plasma evaporation. As already mentioned, in the late rise phase the dynamics 
become much less important and the interplay between cooling and heating processes becomes dominant. The relevant timescale is therefore that reported in Eq. (14).

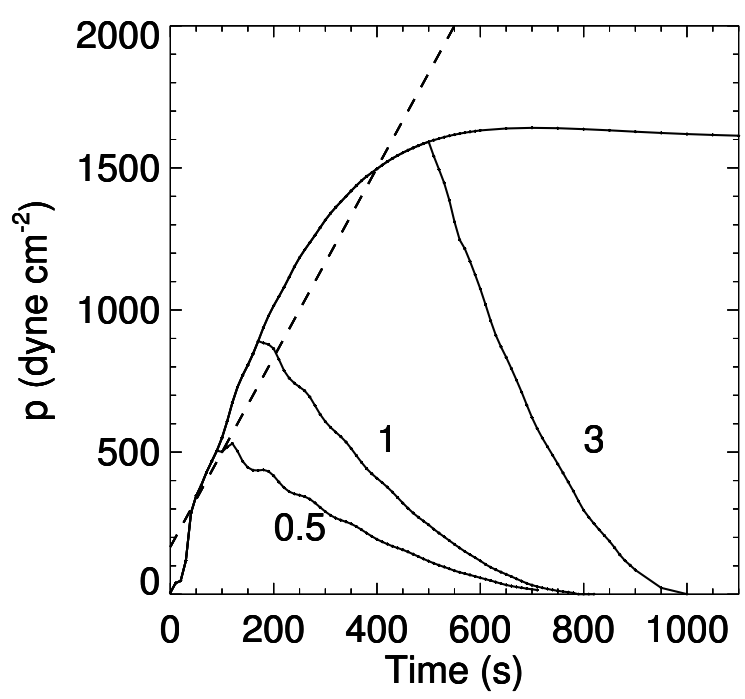

Figure 17: Pressure evolution obtained from a hydrodynamic simulation of a loop strand ignited by heat pulses of different duration (0.5, 1, 3 times the loop decay time, see text) and with a continuous heating. Most of the rise phase can be reasonably described with a linear trend (dashed lines). Image reproduced with permission from Reale (2007), copyright by ESO.

Hydrodynamic simulations confirm that the time required to reach full equilibrium scales as the loop cooling time $\left(\tau_{s}\right)$ and, as shown for instance in Figure 17 (see also Jakimiec et al., 1992), the time to reach flare steady-state equilibrium is:

$$
t_{\mathrm{eq}} \approx 2.3 \tau_{s} .
$$

For $t \geq t_{\text {eq }}$, the density asymptotically approaches the equilibrium value:

$$
n_{0}=\frac{T_{0}^{2}}{2 a^{3} k_{B} L}=1.3 \times 10^{6} \frac{T_{0}^{2}}{L},
$$

where $a=1.4 \times 10^{3}$ (c.g.s. units), or

$$
n_{9}=1.3 \frac{T_{0,6}^{2}}{L_{9}}
$$

If the heat pulse stops before the loop reaches equilibrium conditions, the loop plasma maximum density is lower than the value at equilibrium, i.e., the plasma is underdense (Cargill and Klimchuk, 2004, Section 4.4). Figure 17 shows that, after the initial impulsive evaporation on a timescale given by Eq. (11), the later progressive pressure growth can be approximated with a linear trend. Since the temperature is almost constant in this phase, we can approximate that the density increases linearly for most of the time. We can then estimate the value of the maximum density at the loop apex as:

$$
n_{M} \approx n_{0} \frac{t_{M}}{t_{\mathrm{eq}}},
$$


where $t_{M}$ is the time at which the density maximum occurs.

Phase III ranges between the time at which the heat pulse ends and the time of the density maximum. The latter is also the time at which the decay path crosses the locus of the equilibrium loops (QSS curve). According to Reale (2007), the temperature $T_{M}$ at which the maximum density occurs is:

$$
T_{M}=9 \times 10^{-4}\left(n_{M} L\right)^{1 / 2}
$$

or

$$
T_{M, 6}=0.9\left(n_{M, 9} L_{9}\right)^{1 / 2} .
$$

We can also derive the duration of Phase III, i.e., the time from the end of the heat pulse to the density maximum, as

$$
\Delta t_{0-M} \approx \tau_{c} \ln \psi,
$$

where

$$
\psi=\frac{T_{0}}{T_{M}}
$$

and $\tau_{c}$ [Eq. (12)] is computed for an appropriate value of the density $n_{c}$. A good consistency with numerical simulations is obtained for $n_{c}=(2 / 3) n_{M}$.

By combining Eqs. (20) and (18) we obtain:

$$
\frac{\Delta t_{0-M}}{t_{M}} \approx 1.2 \ln \psi,
$$

which ranges between 0.2 and 0.8 for typical values of $\psi(1.2-2)$.

These scalings are related to the evolution of a single strand under the effect of a local heat pulse. The strands are below the current instrument spatial resolution and, therefore, we have to consider that, if this scenario is valid, we see the envelope of a collection of small scale events. The characteristics of the single heat pulses become, therefore, even more difficult to diagnose, and the question of their frequency, distribution and size remains open. Also from the point of view of the modeling, a detailed description of a multistrand loop implies a much more complex and demanding effort. A possible approach is to literally build a collection of 1-D loop models, each with an independent evolution (Guarrasi et al., 2010). One common approach so far has been to simulate anyhow the evolution of a single strand, and to assume that, in the presence of a multitude of such strands, in the steady state we would see at least one strand at any step of the strand evolution. In other words, a collection of nanoflare-heated strands can be described as a whole with the time-average of the evolution of a single strand (Warren et al., 2002, 2003; Winebarger et al., 2003b,a, see also Section 4.2). Another issue to be explored is whether it is possible, and to what extent, to describe a collection of independently-evolving strands as a single effective evolving loop. For instance, how does the evolution of a single loop where the heating is decreasing slowly compare to the evolution of a collection of independently heated strands, with a decreasing rate of ignition? To what extent do we expect coherence and how is it connected to the degree of global coherence of the loop heating? Is there any kind of transverse coherence or ordered ignition of the strands? It is probably reasonable to describe a multi-stranded loop as a single "effective" loop if we can assume that the plasma loses memory of its previous history. This certainly occurs in late phases of the evolution when the cooling has been going on for a long time.

\subsection{Fine structuring}

The description and role of fine structuring of coronal loops is certainly a challenge for coronal physics, also on the side of modeling, essentially because we have few constraints from observations (Section 3.2.2). Small-scale structuring is already involved in the magnetic carpet scenario and 
flux-tube tectonics model (Priest et al., 2002, see also Section 4.4). One of the first times that the internal structuring of coronal loops have been invoked in a modeling context was for the problem of the interpretation of the uniform filter ratio distribution detected with TRACE along warm loops. Standard models of single hydrostatic loops with uniform heating were soon found to be unable to explain such indication of uniform temperature distribution (Lenz et al., 1999). A uniform filter ratio could be reproduced by the superposition of several thin hydrostatic strands at different temperatures (Reale and Peres, 2000). In alternative, also a model of long loops heated at the footpoints leads to mostly isothermal loops (Aschwanden, 2001). The problem with this model is that footpoint-heated loops (with heating scale height less than $1 / 3$ of the loop half-length) had been shown to be thermally unstable (Mendoza-Briceno and Hood, 1997) and, therefore they cannot be long-lived, as instead observed. A further alternative is to explain observations with steady non-static loops, i.e., with significant flows inside (Winebarger et al., 2001, 2002c, see below). Also this hypothesis does not seem to answer the question (Patsourakos et al., 2004).

A first step to modeling fine-structured loops is to use multistrand static models. Such models show some substantial inconsistencies with observations, e.g., in general they predict too large loop cross sections (Reale and Peres, 2000). Such strands are conceptually different from the thin strands predicted in the nanoflare scenario (Parker, 1988), which imply a highly dynamic evolution due to pulsed-heating. The nanoflare scenario is approached in multi-thread loop models, convolving the independent hydrodynamic evolution of the plasma confined in each pulse-heated strand (see Section 4.3). These are able to match some more features of the evolution of warm loops observed with TRACE (Warren et al., 2002, 2003; Winebarger et al., 2003b,a). According to detailed hydrodynamic loop modeling, an ensemble of independently heated strands can be significantly brighter than a static uniformly heated loop and would have a flat filter ratio temperature when observed with TRACE (Warren et al., 2002). As an extension, time-dependent hydrodynamic modeling of an evolving active region loop observed with TRACE showed that a loop made as a set of small-scale, impulsively heated strands can generally reproduce the spatial and temporal properties of the observed loops, such as a delay between the appearance of the loop in different filters (Warren et al., 2003). An evolution of this approach was to model an entire active region for comparison with a SoHO/EIT observation (Warren and Winebarger, 2006); the modeling includes extrapolating the magnetic field and populating the field lines with solutions to the hydrostatic loop equations assuming steady, uniform heating. The result was the link between the heating rate and the magnetic field and size of the structures, but there were also significant discrepancies with the observed EIT emission.

More recently, modeling a loop system as a collection of thin unresolved strand with pulsed heating has been used to explain why active regions look fuzzier in harder energy bands, i.e. Xrays, and/or hotter spectral lines, e.g., Fe XVI, sensitive to high temperatures ( $\sim 3 \mathrm{MK})$ (Tripathi et al., 2009, Section 3.3.2). Short ( $1 \mathrm{~min})$ pulses with flare-like intensity $(\sim 10 \mathrm{MK})$ are able to produce loops with high filling factors at $\sim 3 \mathrm{MK}$ and lower filling factors at $\sim 1 \mathrm{MK}$ (Guarrasi et al., 2010). The basic reason is that in the dynamic evolution of each strand, the plasma spends a relatively longer time and with a high emission measure at temperature about 3 MK. The consequent prediction that loops should show filamented emission for temperature $>3 \mathrm{MK}$ has received confirmations by observations of active region cores in the $94 \AA$ channel with SDO/AIA (Reale et al., 2011) and in the CaXVII and Fe XVIII EUV lines (Testa and Reale, 2012), although the temperature of the emitting plasma is still debated (Teriaca et al., 2012). Low filling factors of warm loops have been predicted also by full MHD modeling (Dahlburg et al., 2012).

The description of loops as bundles of strands applies also to models that include heating by the dissipation of MHD waves (Alfvén/ion-cyclotron waves - particles). One such model addressed the evidence of flat TRACE/EIT filter-ratios along loops that were explained by the multi-filament loop structure (Bourouaine and Marsch, 2010). Transverse oscillations and flows were observed in multi-stranded loops (Ofman and Wang, 2008; Wang et al., 2012). Multi-stranded loop models 
were used in 3D MHD studies of transverse loop oscillations (e.g., Ofman, 2009) and in MHD normal mode analysis (e.g., Luna et al., 2010).

State-of-the-art approaches to the study of multi-stranded loops are based on the concept that each fibril is independent of the others and that the heating is released randomly presumably with a power-law distribution. Within the limitations of idealized loop models (without magnetic twist, time-dependent thread cross-sections, or oscillation), the coronal loops might then be described as self-organized critical systems with no characteristic timescales (e.g., Bak et al., 1989; Lu and Hamilton, 1991; Charbonneau et al., 2001). This model has had a practical realization specific to reproduce soft X-ray steady-state loops (López Fuentes and Klimchuk, 2010) and is able to reproduced the loop light curves observed, for instance, with GOES/SXI. Another interesting approach is to use artificial neural networks (Tajfirouze and Safari, 2012) as mentioned in Section 3.4.

\section{$4.3 \quad$ Flows}

A generalization of static models of loops (Section 4.1.1) is represented by models of loops with stationary flows, driven by a pressure imbalance between the footpoints (siphon flows). The properties of siphon flows have been studied by several authors (Cargill and Priest, 1980; Priest, 1981; Noci, 1981; Borrini and Noci, 1982; Antiochos, 1984; Thomas, 1988; Montesinos and Thomas, 1989; Noci et al., 1989; Thomas and Montesinos, 1990; Spadaro et al., 1990; Thomas and Montesinos, 1991; Peres et al., 1992; Montesinos and Thomas, 1993). A complete detailed model of loop siphon flows was developed and used to explore the space of the solutions and to derive an extension of RTV scaling laws to loops containing subsonic flows (Orlando et al., 1995b).

Critical and supersonic flows create the conditions for the presence of stationary shocks in coronal loops (Figure 18). The shock position depends on the volumetric heating rate of the loop (Orlando et al., 1995a). The presence of massive flows may alter the line emission with respect to static plasma, because of the delay of the moving plasma to settle to ionization equilibrium (Golub and Herant, 1989). Including the effect of ionization non-equilibrium, the UV lines are predicted to be blue-shifted by loop models (Spadaro et al., 1990). So non-equilibrium emission from flows cannot explain the observed dominant redshifts (Section 3.5). Non-equilibrium of ionization in UV line emission can be driven by shocked siphon flows (Orlando and Peres, 1999) and by reconnection flows (Imada et al., 2011).

In the 1990s, modeling efforts were devoted to explain specifically the extensive evidence of red-shifted UV lines on the solar disk. A hydrodynamic loop model including the effects of nonequilibrium of ionization showed that the redshifts might be produced by downward propagating acoustic waves, possibly stimulated by nanoflares (Hansteen, 1993). Two-dimensional hydrodynamic simulations showed that the UV redshifts might be produced by downdrafts driven by radiatively-cooling condensations in the solar transition region (Reale et al., 1996, 1997b). Predicted redshifts range from those typical of quiet Sun to active regions and may occur more easily in the higher pressure plasma, typical of active regions.

Explosions below the corona were explored to drive flows (Teriaca et al., 1999; Sarro et al., 1999) in magnetic loops around the O VI and C IV formation temperature. The observed redshift of midlow transition region lines as well as the blueshift observed in low coronal lines $\left(T>6 \times 10^{5} \mathrm{~K}\right)$ were compared to numerical simulations of the response of the solar atmosphere to an energy perturbation of $4 \times 10^{24} \mathrm{erg}$, including non-equilibrium of ionization (Teriaca et al., 1999). Performing an integration over the entire period of simulations, they found a redshift in CIV, and a blueshift in O VI and Ne VIII, of a few $\mathrm{km} \mathrm{s}^{-1}$, in reasonable agreement with observations. A similar idea was applied to make predictions about the presence or absence of non-thermal broadening in several spectral lines (e.g., Ne VIII, Mg X, Fe XVII) due to nanoflare-driven chromospheric evaporation (Patsourakos and Klimchuk, 2006). Clearly, the occurrence of such effects in the lines depends considerably on the choice of the heat pulse parameters. Therefore, more constraints are 

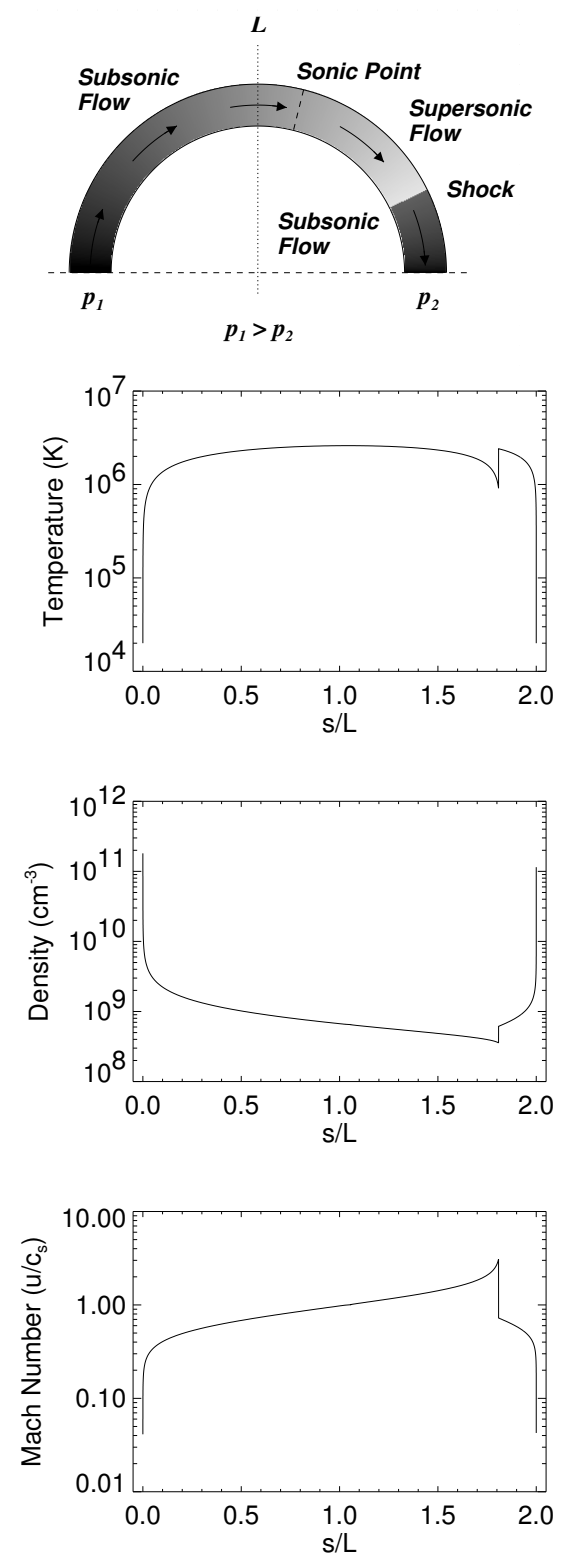

Figure 18: Example of solutions of a siphon flow loop model including a shock. Image reproduced with permission from Orlando and Peres (1999), copyright by Elsevier. 
needed to make the whole model more consistent. In other words, modeling should address specific observations to provide more conclusive results.

Theoretical reasons indicate that flows should be invariably present in coronal loop systems, although they may not be necessarily important in the global loop momentum and energy budget. For instance, it has been shown that the presence of at least moderate flows is necessary to explain why we actually see the loops (Lenz, 2004). The loop emission and detection is in fact due to the emission from heavy ions, like Fe. In hydrostatic equilibrium conditions, gravitational settlement should keep the emitting elements low on the solar surface, and we should not be able to see but the loop footpoints. Instead, detailed modeling shows that flows of few $\mathrm{km} \mathrm{s}^{-1}$ are enough to drag ions high in the corona by Coulomb coupling and to enhance coronal ion abundances by orders of magnitudes. Incidentally, the same modeling shows that, for the same mechanisms, no chemical fractionation of coronal plasma with respect to photospheric composition as a function of the element first ionization potential (FIP) should be present in coronal loops.

Other studies address instead the relative unimportance of flows in coronal loops. In particular, as already mentioned in Section 3.2.2, steady hydrodynamic loop modeling (i.e., assuming equilibrium condition and, therefore, dropping the time-dependent terms in Eqs. (4), (5), and (6)), showed that flows may not be able to explain the evidence of isothermal loops (Patsourakos et al., 2004), as instead proposed by Winebarger et al. (2002c). Flows are able to enhance its density to the levels typically diagnosed from TRACE observations, but they also produce an inversion of the temperature distribution and a structured filter ratio, not observed.

Plasma cooling is a mechanism that may drive significant downflows in a loop (e.g., Bradshaw and Cargill, 2005, 2010). Catastrophic cooling in loops (Müller et al., 2004, 2005) was proposed to explain the evidence of propagating intensity variations observed in the He II $304 \AA$ line with SoHO/EIT (De Groof et al., 2004, Section 3.5). Two possible driving mechanisms had been proposed: slow magnetoacoustic waves or blobs of cool downfalling plasma. A model of cool downfalling blob triggered in a thermally-unstable loop heated at the footpoints gave a qualitative agreement with measured speeds and predicted a significant braking in the high-pressure transition region, to be checked in future high cadence observations in cool lines.

Plasma waves have been more recently proposed to have an important role in driving flows within loops. Acoustic waves excited by heat pulses at the chromospheric loop footpoints and damped by thermal conduction in corona are possible candidates (Taroyan et al., 2005). Even more attention received the propagation of Alfvén waves in coronal loops. Hydrodynamic loop modeling showed that Alfvén waves deposit significant momentum in the plasma, and that steady state conditions with significant flows and relatively high density can be reached (O'Neill and Li, 2005). Analogous results were obtained independently with a different approach: considering a wind-like model to describe a long isothermal loop, Grappin et al. (2003, 2005) showed that the waves can drive pressure variations along the loop which trigger siphon flows. Alfvén disturbances have been more recently shown to be amplified by the presence of loop flows (Taroyan, 2009).

Large-scale MHD models have also addressed the presence of flows in the low corona. These models show that heat pulses released low in the corona in places of strong magnetic field braiding trigger downflows and slight upflows (Figure 19, Hansteen et al., 2010; Zacharias et al., 2011). The corresponding Doppler-shifts are similar to those often observed (see Section 3.5). Most of the mass circulating across the transition region is probably confined in very short loops $\left(\sim 2 \times 10^{8} \mathrm{~cm}\right)$ (Guerreiro et al., 2013).

Large-scale chromospheric upflows (type-II spicules, see Section 3.5) are explored as viable mechanisms of mass and energy supply to coronal loops with loop modeling (Judge et al., 2012). However, theoretical estimates suggest that a corona dominated by this scenario would lead to large discrepancies with observations, therefore confining the possible action of this mechanism in a limited number of structures (Klimchuk, 2012). 

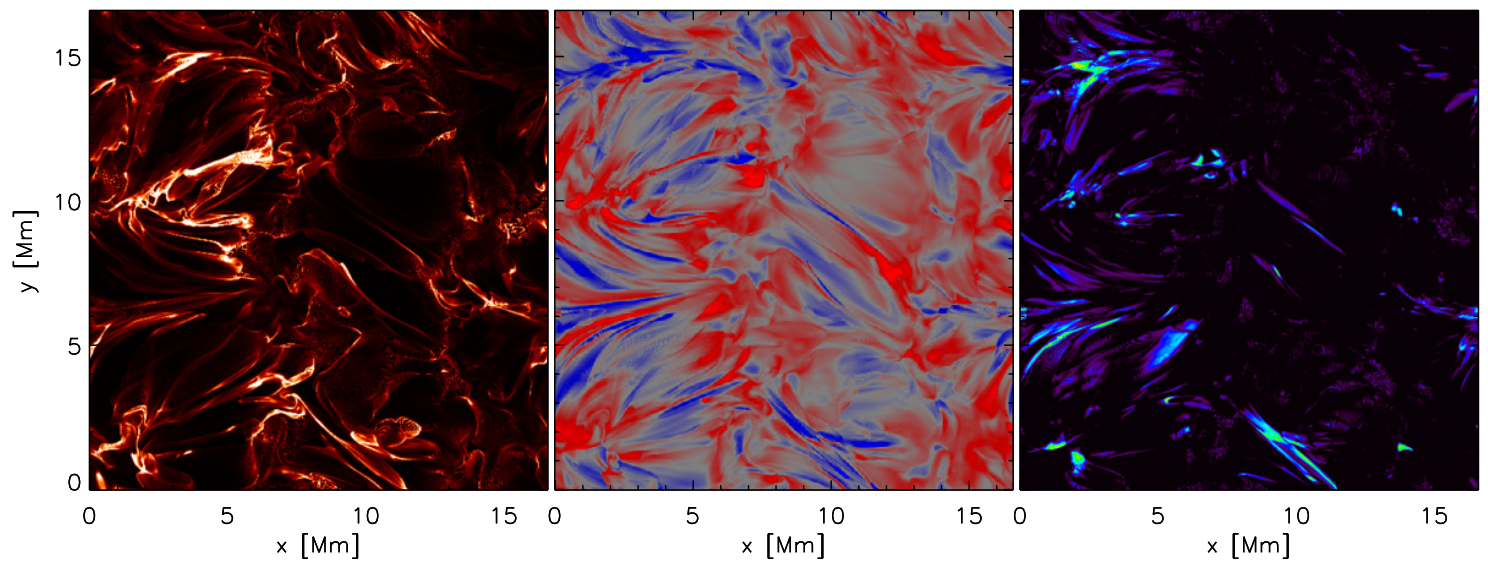

Figure 19: Maps of intensity (left), Doppler shift (middle) and line width (right) in the C IV line in a 3-D MHD simulation of a box of the upper solar atmosphere. The velocity scale is from $-40 \mathrm{~km} \mathrm{~s}^{-1}$ (blue) to $40 \mathrm{~km} \mathrm{~s}^{-1}$ (red). Line widths range from narrow black to wide yellow/red with a maximum of $51 \mathrm{~km} \mathrm{~s}^{-1}$. The average line-shift in the $\mathrm{C}$ IV line is $6.6 \mathrm{~km} \mathrm{~s}^{-1}$ (redshift is positive). Image reproduced with permission from Hansteen et al. (2010), copyright by AAS.

\subsection{Heating}

The problem of what heats coronal loops is essentially the problem of coronal heating, and is a central issue in the whole solar physics. Although the magnetic origin of coronal heating has been well-established since the very first X-ray observations of the corona, the detailed mechanism of conversion of magnetic energy into thermal energy is still under intense debate, because a series of physical effects conspire to make the mechanism intrinsically elusive.

Klimchuk (2006) splits the heating problem into six steps: the identification of the source of energy, its conversion into heat, the plasma response to the heating, the spectrum of the emitted radiation, the final signature in observables. Outside of analytical approaches, the source and conversion of energy are typically studied in detail by means of multi-dimensional full MHD models (e.g., Gudiksen and Nordlund, 2005), which, however, are still not able to provide exhaustive predictions on the plasma response and complete diagnostics on observables. On the other hand, the plasma response is the main target of loop hydrodynamic models, which, instead, are not able to treat the heating problem in a self-consistent way (Section 4.1).

In the investigation of the source of energy, the magnetic field plays an active role in heating the coronal loops (Golub et al., 1980). The observation of a magnetic carpet (Schrijver et al., 1998) suggests that current sheets at the boundary of the carpet cells can dissipate and heat the corona, acting in analogy to geophysical plate tectonics (Priest et al., 2002). A common scenario is that the field lines are wound continuously by the photospheric convective motions and the generated non-potential component is dissipated into heating. Several studies were devoted to the connection and scaling of the magnetic energy to the coronal energy content (Golub et al., 1982) and to the rate of energy release through reconnection (Galeev et al., 1981).

It is known that twisted loops can become kink unstable above a critical twist; however, according to Parker (1988), as soon as the magnetic field lines are tangled at an angle of $\sim 15^{\circ}$, enough magnetic energy can be released to power a loop by rapid reconnection across the spontaneous tangential discontinuities. The dissipation destroys the cross-component of the magnetic field as rapidly as it is produced by the motion of the footpoints, reaching a steady state. The twisting of coronal loops has been studied in the framework of kink instability (Hood and Priest, 1979b; Velli et al., 1990) with resistive effects (Baty, 2000), of loop cross-section (Klimchuk, 2000), of flux emer- 
gence (Hood et al., 2009a), of cellular automaton loop modeling (López Fuentes and Klimchuk, 2010), and in connection with transverse oscillations (Ofman, 2009). Loop twisting or braiding and kink instability can lead to magnetic reconnection with the formation and fragmentation of thin current sheets and their dissipation through resistivity (Hood et al., 2009b; Wilmot-Smith et al., 2010; Bareford et al., 2010; Pontin et al., 2011; Wilmot-Smith et al., 2011; Bareford et al., 2011, 2013).

The comparison of TRACE and Hinode time sequences of active region loops with magnetic field reconstruction models have allowed to measure the changes in the magnetic topology and energy with the time. A high variability corresponds to a high number of magnetic separation lines (Priest et al., 2002) where the energy can be released in short timescales (Lee et al., 2010). A model of nonlinear force-free field traced that magnetic energy is built up in the core of active regions by small-scale photospheric motions (Mackay et al., 2011). The photospheric motions are therefore the ultimate energy source and stress the field or generate waves depending on whether the timescale of the motion is long or short compared to the end-to-end Alfvén travel time.

Following Klimchuk (2006), dissipation of magnetic stresses can be referred to as direct current (DC) heating, and dissipation of waves as alternating current (AC) heating.

The question of the conversion of the magnetic energy into heat is also challenging, because dissipation is predicted to occur on very small scales or large gradients in the corona by classical theory, although there are some indications of anomalously high dissipation coefficients (Martens et al., 1985; Nakariakov et al., 1999; Fuentes-Fernández et al., 2012). As reviewed by Klimchuk (2006), large gradients may be produced in various ways, involving either magnetic field patterns and their evolution, magnetic instabilities such as the kink instability, or velocity pattern, such as turbulence. For waves, resonance absorption and phase mixing may be additional viable mechanisms (see Section 4.4.2).

The problem of plasma response to heating has been kept historically well separated from the primary heating origin, although some attempts have been made to couple them. For instance, in Reale et al. (2005) the time-dependent distribution of energy dissipation along the loop obtained from a hybrid shell model was used as heating input of a time-dependent hydrodynamic loop model (see below). A similar concept was applied to search for signatures of turbulent heating in UV spectral lines (Parenti et al., 2006).

As already mentioned, studies using steady-state or time-dependent purely hydrodynamic loop modeling have addressed primarily the plasma response to heating, and also its radiative emission and the detailed comparison with observations. A forward-modeling including all these steps was performed on a TRACE observation of a brightening coronal loop (Reale et al., 2000a,b, see also Section 3.4). The analysis was used to set up the parameters for the forward modeling, and to run loop hydrodynamic simulations with various assumptions on the heating location and time dependence. The comparison of the TRACE emission predicted by the simulations with the measured one constrained the heat pulse to be short, much less than the observed loop rise phase, and intense, appropriate for a 3 MK loop, and its location to be probably midway between the apex and one of the footpoints.

The investigation of the heating mechanisms through the plasma response is difficult for a variety of reasons. For instance, the problem of background subtraction can be crucial in the comparison with observations, as shown by the three analyses of the same large loop structure observed with Yohkoh/SXT on the solar limb, mentioned in Section 3.3. More specifically, Priest et al. (2000) tried to deduce the form of the heating from Yohkoh observed temperature profiles and found that a uniform heating best describes the data, if the temperature is obtained from the ratio of the total filter intensities, with no background subtraction. Aschwanden (2001) split the measured emission into two components and found a better agreement with heating deposited at the loop footpoints. Reale (2002b) revisited the analysis of the same loop system, considering conventional hydrostatic single-loop models and accounting accurately for an unstructured background 
contribution. With forward-modeling, i.e., synthesizing from the model observable quantities to be compared directly with the data, background-subtracted data are fitted with acceptable statistical significance by a model of relatively hot loop $(\sim 3.7 \mathrm{MK})$ heated at the apex, but it was pointed out the importance of background subtraction and the necessity of more specialized observations to address this question. More diagnostic techniques to compare models with observations were proposed afterwards (e.g., Landi and Landini, 2005).

Independently of the adopted numerical or theoretical tool, many studies have been addressing the mechanisms of coronal loop heating clearly distinguishing between the two main classes, i.e., DC heating through moderate and frequent explosive events, named nanoflares (e.g., Parker, 1988) and AC heating via Alfvén waves (e.g., Litwin and Rosner, 1998).

\subsubsection{DC heating}

Heating by nanoflares has a long history as a possible candidate to explain the heating of the solar corona, and, in particular, of the coronal loops (e.g., Peres et al., 1993; Cargill, 1993; Kopp and Poletto, 1993; Shimizu, 1995; Judge et al., 1998; Mitra-Kraev and Benz, 2001; Katsukawa and Tsuneta, 2001; Mendoza-Briceño et al., 2002; Warren et al., 2002, 2003; Spadaro et al., 2003; Cargill and Klimchuk, 1997, 2004; Müller et al., 2004; Testa et al., 2005; Reale et al., 2005; Taroyan et al., 2006; Vekstein, 2009). The coronal tectonics model (Priest et al., 2002) is an updated version of Parker's nanoflare theory, for which the motions of photospheric footpoints continually build up current sheets along the separatrix boundaries of the flux coming from each microscopic source (Priest, 2011).

Models of loops made of thousands of nanoflare-heated strands were developed and applied to derive detailed predictions (Cargill, 1994). In particular, whereas the loop total emission measure distribution should steepen above the canonical $T^{1.5}$ (Jordan, 1980; Orlando et al., 2000; Peres et al., 2001) dependence for temperature above 1 MK. Moreover, it was stressed the importance of the dependence of effects such as the plasma dynamics (filling and draining) on the loop filling factor driven by the elemental heat pulse size (Section 4.1.2). The nanoflare model was early applied to the heating of coronal loops observed by Yohkoh (Cargill and Klimchuk, 1997). A good match was found only for hot (4 MK) loops, with filling factors less than 0.1 , so that it was hypothesized the existence of two distinct classes of hot loops.

Although there is evidence of intermittent heating episodes, it has been questioned whether and to what extent nanoflares are able to provide enough energy to heat the corona (e.g., Aschwanden, 1999). On the other hand, loop models with nanoflares, and, in particular, those considering a prescribed random time distribution of the pulses deposited at the footpoints of multi-stranded loops have been able to explain several features of loop observations, for instance, of warm loops from TRACE (Warren et al., 2002, 2003, see Section 3.2.2).

Hydrodynamic loop modeling showed also that different distributions of the heat pulses along the loop have limited effects on the observable quantities (Patsourakos and Klimchuk, 2005), because most of the differences occur at the beginning of the heat deposition, when the emission measure is low, while later the loop loses memory of the heat distribution (see also Winebarger and Warren, 2004). An application of both static and impulsive models to solar active regions showed that the latter ones are able to simultaneously reproduce EUV and SXR loops in active regions, and to predict radial intensity variations consistent with the localized core and extended emissions (Patsourakos and Klimchuk, 2008). As a further improvement, the simulation of an entire active region with an impulsive heating model reproduced the total observed soft X-ray emission in all of the Yohkoh/SXT filters (Warren and Winebarger, 2007). However, once again, at EUV wavelengths the agreement between the simulation and the observation was only partial.

Nanoflares have been studied also in the framework of stellar coronae. Intermittent heating by relatively intense nanoflares deposited at the loop footpoints makes the loop stable on long 
timescales (Testa et al., 2005; Mendoza-Briceño et al., 2005) (loops infrequently heated at the footpoints are unstable) and, on the other hand, produces a well-defined peak in the average DEM of the loop, similar to that derived from the DEM reconstruction of active stars (Cargill, 1994; Testa et al., 2005). Therefore, this is an alternative way to obtain a steep temperature dependence of the loop emission measure distribution in the low temperature range.

An alternative approach to study nanoflare heating is to analyze intensity fluctuations (Shimizu and Tsuneta, 1997; Vekstein and Katsukawa, 2000; Katsukawa and Tsuneta, 2001; Vekstein and Jain, 2003) and to derive their occurrence distribution (Sakamoto et al., 2008, 2009). From the width of the distributions and autocorrelation functions, it has been suggested that nanoflare signatures are more easily found in observations of warm TRACE loops than of hot Yohkoh/SXT loops. It is to be investigated whether the results change after relaxing the assumption of temperatureindependent distribution widths. Also other variability analysis of TRACE observations was found able to put constraints on loop heating. In particular, in TRACE observations, the lack of observable warm loops and of significant variations in the moss regions implies that the heating in the hot moss loops should not be truly flare-like, but instead quasi-steady and that the heating magnitude is only weakly varying (Antiochos et al., 2003; Warren et al., 2010b).

An analogous approach is to analyze the intensity distributions. The distribution of impulsive events vs their number in the solar and stellar corona is typically described with a power law. The slope of the power law is a critical parameter to establish weather such events are able to heat the solar corona (Hudson, 1991). In particular, a power law index of 2 is the critical value above or below which flare-like events may be able or unable, respectively, to power the whole corona (e.g., Aschwanden, 1999; Bareford et al., 2010; Tajfirouze and Safari, 2012). Unfortunately, due to the faintness of the events, the distribution of weak events is particularly difficult to derive and might even be separate from that of proper flares and microflares. A hydrodynamic model was used to simulate the UV emission of a loop system heated by nanoflares on small, spatially unresolved scales (Parenti and Young, 2008). The simulations confirmed previous results that several spectral lines have an intensity distribution that follows a power-law, in a similar way to the heating function (Hudson, 1991). However, only the high temperature lines best preserve the heating function's power law index (especially Fe XIX).

The shape of the emission measure distribution is, in principle, a powerful tool to constrain the heating mechanisms. The width in temperature provides information about the temporal distribution of a discontinuous heating mechanism: for a broad (multi-thermal) distribution the simultaneous presence of many temperature components along the line of sight may be produced by many strands randomly heated for a short time and then spending most of the time in the cooling, thus "crossing" many different temperatures. A peaked distribution, i.e., plasma closer to an isothermal condition, indicates a plasma sustained longer at a certain temperature, with a heating much more uniform in time than for multi-thermal loops. A semi-analytical loop model of a cycling heating/cooling (Cargill and Klimchuk, 2004) naturally led to hot-underdense/warmoverdense loop (Section 4.1.2), as observed (Winebarger et al., 2003b, Section 3.3.3), and showed that the width of the DEM of a nanoflare-heated loop can depend on the number of strands which compose the loop: a relatively flat DEM or a peaked (isothermal) DEM are obtained with strands of diameter about $15 \mathrm{~km}$ or about $150 \mathrm{~km}$, respectively. This is of relevance for the diagnostics both of the loop fine structure (Section 3.2.2) and of the DEM reconstruction (Section 3.3). In general, a broad emission measure distribution would be a signature of a low-frequency heating, whereas a peaked distribution would be a signature of high-frequency heating (Warren et al., 2010b; Susino et al., 2010). The timescale is basically dictated by the cooling times. High frequency heating seems to explain several debated evidence in warm loops of active regions, i.e., loop lifetime, high density, and the narrow differential emission measure, but not the higher temperature loops detected in the X-rays (Warren et al., 2010a). It is remarked that overdense plasma would be emphasized also by deviations from equilibrium of ionization due to impulsive heating (Bradshaw and Klimchuk,

Living Reviews in Solar Physics

http: //www. livingreviews . org/lrsp-2014-4 
2011), and that the predicted cool side of the emission measure distribution might steepen using updated radiative losses (Reale et al., 2012). However, the constraints on heating from emission measure distribution are largely debated; broad and peaked emission measure distributions of hot 3 MK loops might be compatible with steady heating models (Winebarger et al., 2011). This debate has been specifically addressed and all evidence has been collected and analysed through loop modeling. In particular, the consistency of the DEM slopes on the cool side with low frequency nanoflare heating has been tested. It has been found that, although heating by single pulses might explain the majority of DEMs derived in the literature (Bradshaw et al., 2012) and that trains of nanoflares might explain practically all of them (Reep et al., 2013), the uncertainties in the data analysis and DEM reconstruction are too large reach conclusive answers. Radiative losses are important to the existence of small and cool loops (height $\leq 8 \mathrm{Mm}, \mathrm{T} \leq 10^{5} \mathrm{~K}$ ) that determine the cool side of the emission measure distribution (Sasso et al., 2012).

Support to dynamic heating comes from modeling loops with steady heating located at the footpoints. It is known that such heating is not able to keep loop atmosphere in steady equilibrium because they are thermally unstable (Antiochos and Klimchuk, 1991; Antiochos et al., 1999; Müller et al., 2004; Karpen and Antiochos, 2008; Mok et al., 2008). Catastrophic cooling occurs along the loops some time after the heating is switched on and might explain deviations from hydrostatic equilibrium, and some features of the light curves measured in the EUV band (Peter et al., 2012). However, the timescales required by this scenario seem too long compared to the measured loop lifetimes (Klimchuk et al., 2010).

\subsubsection{AC heating}

Loop oscillations, modes and wave propagation deserve a review by themselves, and are outside of the scope of the present one. Here we account for some aspects which are relevant for the loop heating. A review of coronal waves and oscillations can be found in Nakariakov and Verwichte (2005). New observations from SDO AIA provide ample evidence of wave activity in the solar corona (Title, 2010), as reported on in Section 3.5.2.

As reviewed by Klimchuk (2006), MHD waves of many types are generated in the photosphere, e.g., acoustic, Alfvén, fast and slow magnetosonic waves. Propagating upwards, the waves may transfer energy to the coronal part of the loops. The question is what fraction of the wave flux is able to pass through the very steep density and temperature gradients in the transition region. Acoustic and slow-mode waves form shocks and are strongly damped, fast-mode waves are strongly refracted and reflected (Narain and Ulmschneider, 1996).

Ionson $(1978,1982,1983)$ devised an LRC equivalent circuit to show the potential importance of AC processes to heat the corona. Hollweg (1984) used a dissipation length formalism to propose resonance absorption of Alfvén waves as a potential coronal heating mechanism. A loop may be considered as a high-quality resonance cavity for hydromagnetic waves. Turbulent photospheric motions can excite small-scale waves. Most Alfvén waves are strongly reflected in the chromosphere and transition region, where the Alfvén speed increases dramatically with height. Significant transmission is possible only within narrow frequency bands centered on discrete values where loop resonance conditions are satisfied (Hollweg, 1981, 1984; Ionson, 1982). The waves resonate as a global mode and dissipate efficiently when their frequency is near the local Alfvén waves frequency $\omega_{A} \approx 2 \pi v_{A} / L$. By solving the linearized MHD equations, Davila (1987) showed that this mechanism can potentially heat the corona, as further supported by numerical solution of MHD equations for low beta plasma (Steinolfson and Davila, 1993), and although Parker (1991) argued that Alfvén waves are difficult to be generated by solar convection.

Hollweg (1985) estimated that enough flux may pass through the base of long $\left(>10^{10} \mathrm{~cm}\right)$ active region loops to provide their heating, but shorter loops are a problem, since they have higher resonance frequencies and the photospheric power spectrum is believed to decrease exponentially 
with frequency in this range. Litwin and Rosner (1998) suggested that short loops may transmit waves with low frequencies, as long as the field is sufficiently twisted. Hollweg and Yang (1988) proposed that Alfvén resonance can pump energy out of the surface wave into thin layers surrounding the resonant field lines and that the energy can be distributed by an eddy viscosity throughout large portions of coronal active region loops.

Waves may be generated directly in the corona, and some evidence was found (e.g., Nakariakov et al., 1999; Aschwanden et al., 1999a; Berghmans and Clette, 1999; De Moortel et al., 2002). It is unclear whether coronal waves carry a sufficient energy flux to heat the plasma (Tomczyk et al., 2007). Ofman et al. (1995) studied the dependence on the wavenumber for comparison with observations of loop oscillations and found partial agreement with velocity amplitudes measured from non-thermal broadening of soft X-ray lines. The observed non-thermal broadening of transition region and coronal spectral lines implies fluxes that may be sufficient to heat both the quiet Sun and active regions, but it is unclear whether the waves are efficiently dissipated (Porter et al., 1994). Furthermore, the non-thermal line broadening could be produced by unresolved loop flows that are unrelated to waves (e.g., Patsourakos and Klimchuk, 2006). Ofman et al. (1998) included inhomogeneous density structure and found that a broadband wave spectrum becomes necessary for efficient resonance and that it fragments the loop into many density layers that resemble the multistrand concept. The heat deposition by the resonance of Alfvén waves in a loop was investigated by O'Neill and Li (2005). A multi-strand loop model where the heating is due to the dissipation of MHD waves was applied to explain filter-ratios along loops (Bourouaine and Marsch, 2010, see Sections 3.3.3, 4.2). By assuming a functional form first proposed by Hollweg (1986), hydrodynamic loop modeling showed that, depending on the model parameters, heating by Alfvén waves leads to different classes of loop solutions, such as the isothermal cool loops indicated by TRACE, or the hot loops observed with Yohkoh/SXT. Specific diagnostics are still to be defined for the comparison with observations.

Efficient wave dissipation may be allowed by enhanced dissipation coefficients inferred from fast damping of flaring loop oscillations in the corona (Nakariakov et al., 1999), but the same effect may also favor efficient magnetic reconnection in nanoflares. Alfvén waves required for resonant absorption are relatively high frequency waves. Evidence for lower frequency Alfvén waves has been found in the chromosphere with the Hinode SOT (De Pontieu et al., 2007b). Such waves may supply energy in the corona even outside of resonance with different mechanisms to be explored with modeling. Among dissipation mechanisms phase mixing with enhanced resistivity was suggested by Ofman and Aschwanden (2002) and supported by the analysis of Ofman and Wang (2008). Also multistrand structure has been recognized to be important in possible wave dissipation and loop twisting (Ofman, 2009). Long-period (> $10 \mathrm{~s}$ ) chromospheric kink waves might propagate into the corona by transformation into Alfvén waves and be dissipated there (Soler et al., 2012).

In the more general context of coronal heating, after several previous works, follow-up modeling and analytical effort has been devoted to the dissipation of Alfven waves through phase mixing (e.g., Heyvaerts and Priest, 1983; Nakariakov et al., 1997; Botha et al., 2000; Ofman and Aschwanden, 2002) and ponderomotive force (Verwichte et al., 1999) in a nonideal inhomogeneous medium, finding effects on very long timescales (> 1 month, McLaughlin et al., 2011).

Intensity disturbances propagating along active region loops at speeds above $100 \mathrm{~km} \mathrm{~s}^{-1}$ were detected with TRACE and interpreted as slow magnetosonic waves (Nakariakov et al., 2000). These waves probably originate from the underlying oscillations, i.e., the 3-minute chromospheric/transition-region oscillations in sunspots and the 5-minute solar global oscillations (p-modes). Slow magnetosonic waves might be good candidates as coronal heating sources according to a detailed model, including the effect of chromosphere and transition region and of the radiative losses in the corona (Beliën et al., 1999). Such waves might be generated directly from upward propagating Alfvén waves. Contrary conclusions, in favor of fast magnetosonic waves, have been also obtained, but with much simpler modeling (Pekünlü et al., 2001). Slow magnetosonic 
waves with periods of about 5 minutes have been more recently detected in the transition region and coronal emission lines by Hinode/EIS at the footpoint of a coronal loop rooted at plage, but found to carry not enough energy to heat the corona (Wang et al., 2009). Slow magnetosonic waves might be coupled to upflows and produced by impulsive events at the base of active region loops (Ofman et al., 2012).

Investigation of AC heating has been made also through comparison with DC heating. Antolin et al. (2008) compared observational signatures of coronal heating by Alfvén waves and nanoflares using two coronal loop models and found that Hinode XRT intensity histograms display powerlaw distributions whose indices differ considerably, to be checked against observations. Lundquist et al. $(2008 \mathrm{a}, \mathrm{b})$ applied a method for predicting active region coronal emissions using magnetic field measurements and a chosen heating relationship to 10 active regions. With their forwardmodeling, they found a volumetric coronal heating rate proportional to magnetic field and inversely proportional to field-line loop length, which seems to point to, although not conclusively, the steadystate scaling of two heating mechanisms: van Ballegooijen's current layers theory (van Ballegooijen, 1986), taken in the AC limit, and Parker's critical angle mechanism (Parker, 1988), in the case where the angle of misalignment is a twist angle. As interesting points of contacts with the models of impulsive heating, it has been proposed that loops can be heated impulsively by Alfvén waves dissipated on reasonable timescales through turbulent cascade that develops when the waves are transmitted from the photosphere to the corona (van Ballegooijen et al., 2011; Asgari-Targhi et al., 2013), using reduced MHD equations.

\subsection{Large-scale modeling}

Coronal loops have been studied also with models that include the magnetic field. We can distinguish several levels of treatment of the magnetic effects. One basic level is to use global scalings to discriminate between different heating mechanisms. Based on a previous study of the plasma parameters and the magnetic flux density (Mandrini et al., 2000), Démoulin et al. (2003) derived the dependence of the mean coronal heating rate on the magnetic flux density from the analysis of an active region. By using the scaling laws of coronal loops, they found that models based on the dissipation of stressed, current-carrying magnetic fields are in better agreement with the observations than models that attribute coronal heating to the dissipation of MHD waves injected at the base of the corona. A similar approach was applied to the whole corona, by populating magnetic field lines taken from observed magnetograms with quasi-static loop atmospheres (Schrijver et al., 2004). The best match to X-ray and EUV observation was obtained with a heating that scales as expected from DC reconnection at tangential discontinuities.

Large-scale modeling has been able to explain the ignition of warm loops from primary energy release mechanisms. A large-scale approach (see also Section 4.1) is by "ab initio" modeling, i.e., with full MHD modeling of an entire coronal region (Gudiksen and Nordlund, 2005; Gudiksen et al., 2011). Observed solar granular velocity pattern, a potential extrapolation of a SoHO/MDI magnetogram, and a standard stratified atmosphere are used as initial conditions. The first simulations showed that, at steady state, the magnetic field is able to dissipate $(3-4) \times 10^{6} \mathrm{erg} \mathrm{cm}^{-2} \mathrm{~s}^{-1}$ in a highly intermittent corona, at an average temperature of $\sim 10^{6} \mathrm{~K}$, adequate to reproduce typical warm loop populations observed in TRACE images. Warm loops were also obtained with time-dependent loop modeling including the intermittent magnetic dissipation in MHD turbulence due to loop footpoint motions (Reale et al., 2005). The dissipation rate along a loop predicted with a hybrid-shell model (Nigro et al., 2004) was used as heating input [see Eq. (6)] in a proper time-dependent loop model, the Palermo-Harvard code (Peres et al., 1982). It was shown that the most intense nanoflares excited in an ambient magnetic field of about $10 \mathrm{G}$ can produce warm loops with temperatures of $1-1.5 \mathrm{MK}$ in the corona of a $30000 \mathrm{~km}$ long loop.

More recently, reduced MHD (rMHD) was used to identify MHD anisotropic turbulence as the 
physical mechanism responsible for the transport of energy from the large scales, where energy is injected by photospheric motions, to the small scales, where it is dissipated (Rappazzo et al., 2007, 2008). Strong turbulence was found for weak axial magnetic fields and long loops. The predicted heating rate is appropriate for warm loops, in agreement with Reale et al. (2005). Shell models of rMHD turbulence were used to analyze the case of a coronal loop heated by photospheric turbulence and found that the Alfvén waves interact nonlinearly and form turbulent spectra (Buchlin and Velli, 2007). An intermittent heating function is active, on average able to sustain the corona and proportional to the aspect ratio of the loop to the $\sim 1.5$ power. Adding a profile of density and/or magnetic field along the loop somewhat change the heat deposition, in particular in the low part of the loop (Buchlin et al., 2007). These models also predict the formation of current sheets that can be dissipated on these small scales and impulsively through turbulent cascades (Rappazzo et al., 2010; Rappazzo and Velli, 2011). Transient current sheets are also found from large-scale full MHD modeling (Bingert and Peter, 2011). In the same framework loops have been described as partially resonant cavities for low-frequency fluctuations transmitted from the chromosphere (Verdini et al., 2012).

There are new efforts to include magnetic effects in the loop modeling. Haynes et al. (2008) studied observational properties of a kink unstable coronal loop, using a fluid code and finding potentially observable density effects. Browning et al. (2008) studied coronal heating by nanoflares triggered by a kink instability using three-dimensional magnetohydrodynamic numerical simulations of energy release for a cylindrical coronal loop model. Magnetic energy is dissipated, leading to large or small heating events according to the initial current profile.

Interesting perspectives are developing from models in which self-organized criticality triggers loop coronal heating (e.g., López Fuentes and Klimchuk, 2010). For Uzdensky (2007) and Cassak et al. (2008) coronal heating is self-regulating and keeps the coronal plasma roughly marginally collisionless. In the long run, the coronal heating process may be represented by repeating cycles that consist of fast reconnection events (i.e., nanoflares), followed by rapid evaporation episodes, followed by relatively long periods $(\sim 1 \mathrm{hr})$ during which magnetic stresses build up and the plasma simultaneously cools down and precipitates. An avalanche model was proposed for solar flares (Morales and Charbonneau, 2008), based on an idealized representation of a coronal loop as a bundle of magnetic flux strands wrapping around one another. The system is driven by random deformation of the strands, and a form of reconnection is assumed to take place when the angle subtended by two strands crossing at the same lattice site exceeds some preset threshold. For a generic coronal loop of length $10^{10} \mathrm{~cm}$ and diameter $10^{8} \mathrm{~cm}$, the mechanism leads to flare energies ranging between $10^{23}$ and $10^{29} \mathrm{erg}$, for an instability threshold angle of 11 degrees between contiguous magnetic flux strands. 


\section{$5 \quad$ Stellar Coronal Loops}

Non-solar X-ray missions since Einstein and European X-ray Observatory SATellite (EXOSAT) have established that most other stars have a confined corona, often much more active that the solar one (e.g., Linsky and Serio, 1993). The level of activity is ruled by several factors, but, first of all, the age of the star is important: young fast-rotating stars are more active (e.g., Telleschi et al., 2005). The topic of stellar coronal loops deserves a review by itself (e.g., Rosner et al., 1985) and here only a few relevant issues are discussed. A complete and more recent review of stellar coronae, with an extensive part regarding loops, is by Güdel (2004). In the framework of the solar-stellar connection, it is very important the comparison of what we know about the spatially resolved but single solar corona and what about the unresolved but numerous stellar coronae, which offer a variety of different environments. The lack of spatial resolution inhibits to obtain direct information about the size and appearance of the loops, and the general aspect of the corona. We therefore have to rely on indirect evidence. One possible approach to get information is to benefit from transient X-ray events, such as flares, which provide estimations of the loop scale length from their dependence on the decay and rise timescales (Reale, 2002a, 2003, and Section 4.1.2 for reviews). Detailed hydrodynamic modeling can provide even more constraints, for instance, on the heat deposition (e.g., Reale et al., 1988, 2004). The study of stellar X-ray flares allowed, for instance, to constrain that most stellar flares involve plasma confined in closed structures (Reale et al., 2002), and to infer the presence both of loops with size similar to those observed on the Sun (e.g., Reale et al., 1988) and of giant loops (Favata et al., 2005; Getman et al., 2008), with length exceeding the stellar radius. Analogies between solar and stellar downflows have also been found (Reale et al., 2013). Radio observations have provided direct evidence for the presence of large coronal loops in the Algol system (Peterson et al., 2010).

Another approach is to use the entire solar X-ray corona as a template and "Rosetta stone" to interpret stellar coronae. A detailed implementation of this approach was devised and applied extensively using Yohkoh data over its entire life, which covers a whole solar cycle (Orlando et al., 2000; Peres et al., 2000). It was shown that the solar corona indeed provides a pattern of components, i.e., quiet structures, active regions, active region cores, flares, which can be identified in stellar coronal data and which can explain stellar activity giving different weights to the components (Peres et al., 2001, 2004). The method was also applied to describe stellar coronae in terms of loop populations and to extract general information and constraints on coronal heating (Peres et al., 2004). It was applied to flares (Reale et al., 2001) and to describe the evolution of active regions (Orlando et al., 2004). More recently it was shown that a continuous unresolved flaring activity may explain the most active coronae, but also that the coronal heating appears to follow different scaling for quiet regions and for active and flaring regions across the cycle (Argiroffi et al., 2008).

Cargill and Klimchuk (2006) realized that the strong hot peaks in the emission measuretemperature distributions in the coronae of some binary stars (Sanz-Forcada et al., 2003) are similar to those expected for an impulsively-heated solar corona. A coronal model comprised of many impulsively heated strands shows that the evidence may be compatible with coronae made of many very small loops (length under $10^{3} \mathrm{~km}$ ) heated by microflares.

The growing evidence of hot plasma and of variability will link more tightly the investigation of the solar coronal heating to the study of stellar coronae, where very hot steady components are often detected (e.g., Schmitt et al., 1990; Scelsi et al., 2005). 


\section{Conclusions and Perspectives}

Coronal loops have been the subject of in-depth studies for over 50 years. Since they owe their identity to the brightness of the confined plasma, most of the studies have addressed the physics of the confined plasma, i.e., its structure, dynamics, and evolution. The coronal loops are the building blocks of the bright solar corona and, as such, they are important as the basic laboratory to investigate the mechanisms of coronal magnetism, dynamics, and heating. Our knowledge on coronal loops has progressed with the development of the instrument capabilities. The starting point has been the observation, mostly in the X-ray band, that coronal loops are globally steady on timescales longer than the plasma cooling times. This has allowed to develop the loop scaling laws, which work well to describe the hydrostatic properties of coronal plasma confined in a magnetic flux tube (Section 4.1.1). However, since the 1990s observations have more and more revealed that coronal loops are dynamic and structured, both spatially and thermally. Imaging instruments have shown bundles of fine strands whose thickness is not well resolved up-to-date, although we begin to have constraints from the latest observations with the Hi-C $(\sim 100 \mathrm{~km})$. The lack of agreement about the thickness values might indicate a distribution of strand sizes (Section 3.2.2). EUV spectroscopy shows a variety of thermal structuring, from nearly isothermal loops to broad distributions over a temperature decade $(6<\log T<7)$. The width of the distributions might depend on the heating intensity (warm vs hot loops), on the age of the active region and/or on the magnetic field (Section 3.3). The presence, whenever confirmed, of minor but widespread and filamented, very hot plasma components $(\log T \sim 7)$ out of flares might be a tracer of smallscale impulsive heating. Coronal rain might mark thermal instability on small scales of thickness. High-resolution spectroscopy has also detected widespread flows $\left(\sim 10 \mathrm{~km} \mathrm{~s}^{-1}\right)$ with a complex pattern, especially low in the corona and transition region (Section 3.5). The pattern is described quite well as switching from redshifts to blueshifts with increasing temperature, thus indicating the coexistence of cool downfalling plasma and warm evaporation. This complex pattern is also a signature of fine structure, at least in the low corona. Higher in the corona, flow patterns are less defined, simply because of geometrical reasons. Fast upflows $\left(\sim 100 \mathrm{~km} \mathrm{~s}^{-1}\right)$ from the chromosphere might play a role in feeding coronal loops (Section 3.5.1). Continuous monitoring of the full solar disk has also allowed very detailed photometric analysis (Section 3.4). The detailed analysis of the light curves seems to indicate the presence of systematic cooling patterns, both in the X-rays and, from comparison of different channels, in the EUV band. The monitoring has also allowed the detection of MHD waves propagating along coronal loops (Section 3.5.2).

The physics of the plasma confined inside coronal loops is well described with compressible hydrodynamics with a very important role of thermal conduction and radiative losses from optically thin medium. The mass and energy are transported mostly along the field lines, with little direct role of the magnetic field. The initial hydrostatic single loop scenario is now replaced by a much more dynamic one with loops structured into bundle of finer strands (Section 4.1.2). The physics of the plasma inside each strand does not change much, but one important issue becomes how these strands combine into the unresolved observed structures. Multi-strand loop modeling is able to explain the fuzzier and fuzzier appearance of loops observed in channels sensitive to hotter and hotter emission, and flat filter ratios along loops (Section 4.2). A proper MHD description is required when we need to address directly the interaction of plasma with the ambient magnetic field, both regarding the confinement itself, e.g., the expansion across the transition region, and the conversion of the magnetic energy to power the plasma temperature and dynamics. Although flows along loops have long been modeled (Section 4.3), the recent evidence for upflows from the chromosphere has provided a new boost to models and, since it has been suggested that the flows might also carry the energy to heat the loop plasma, it has also revitalized the investigation of coronal heating, the central issue of coronal loop physics. The most basic question regarding the conversion of magnetic energy into heat remains probably whether this conversion occurs on small

Living Reviews in Solar Physics

http: //www. livingreviews . org/lrsp-2014-4 
or large timescales, both in the charging and in the release (Section 4.4). This may make the difference between energy provided directly through fast magnetic reconnection (nanoflares) and energy dissipated more gradually by MHD waves, but new modeling seems to make the boundary much more blurred, wheareas MHD waves are dissipated impulsively through turbulent cascades. So, at the moment, there is some convergence to heating mechanisms released on short timescales inside thin strands composing the loops. The charge-and-release processes are also deserving attention regarding both the connection to the magnetic field, and the cadence and distribution of the events in the single strand. Turbulent cascades might provide a natural explanation for the dissipation of magnetic energy in anomalously small scales.

Key issues for the future remain the loop fine structure and dynamics. We need to address what are the ultimate elementary loop components, and whether they are unique or determined by local conditions, i.e., what determines the section scale size. We need the highest possible spatial resolution, probably in different bands. Further investigation of the plasma fine thermal structure and dynamics requires also high spectral resolution. High resolution broad-band X-ray spectroscopy is foreseeable to probe the hot components, signature of impulsive heating. Also the investigation of temporal variations still deserves attention. If loops are really so dynamic and subject to a distribution of heating events, whatever it is, the light curves are very difficult to interpret and signatures of any possible small-scale events are confused in a storming activity and by the plasma inertia. The analysis of emission variations is very important, because it can potentially shed light on heating mechanisms based on short impulsive events (nanoflares) or on wave-like phenomena (Alfvén waves). The continuous monitoring by the SDO mission is a very powerful tool for temporal variability studies. The analysis of imaging multi-channel observations seems to indicate widespread plasma cooling, which needs further independent investigation. Observations from IRIS are providing very detailed information about the interaction of the corona with the chromosphere and, in particular, about the intriguing transition region. Highest-resolution observations in the EUV seem also to emphasize the importance of the transition region, because it is very sensitive to changes of the physical conditions and, therefore, it can be a tracer of basic loop processes.

Great help is expected from modeling. Multi-stranded time-dependent loop models are still providing a wealth of information and might contribute to study specific issues such as the sequence and the relative weight of evaporation-draining cycles, and the vertical structure and dynamics of the thin transition region. The coupling with the chromosphere is becoming increasingly important. Improving numerical and computing resources are allowing to address the more basic question of the coupling of the plasma with the magnetic field and of the conversion of the magnetic energy into heat. The approaches involve both full MHD models and other models that couple different regimes, such as the large-scale magnetic field and the locally confined plasma. A totally selfconsistent description is still out-of-reach, but 3D MHD models are beginning to attack some very basic issues, regarding the heat release, such as the role of the MHD instabilities and their switchon. Turbulence seems a key to the anomalous dissipation needed to explain the loop ignition.

The study of coronal loops is very alive and is the subject of Coronal Loop Workshops, taking place every two years, which are site of debate, inspiration of new investigations, and school for young investigators. 


\section{Acknowledgements}

The author thanks P. Testa, S. Orlando, G. Peres, J. Klimchuk, and the anonymous referees for suggestions. The author acknowledges support from the Italian Ministero dell'Università e della Ricerca. The Transition Region and Coronal Explorer (TRACE) is a mission of the StanfordLockheed Institute for Space Research, and part of the NASA Small Explorers program. The solar X-ray images of Figure 2 is from the Yohkoh mission of ISAS, Japan. The X-ray Telescope was prepared by the Lockheed-Martin Solar and Astrophysics Laboratory, the National Astronomical Observatory of Japan, and the University of Tokyo with the support of NASA and ISAS. SDO is the first mission launched for NASA's Living With a Star program. 


\section{References}

Acton, L. W., Finch, M. L., Gilbreth, C. W. et al., 1980, "The soft X-ray polychromator for the Solar Maximum Mission", Solar Phys., 65, 53-71. [DOI], [ADS]. (Cited on page 8.)

Antiochos, S. K., 1979, "The stability of solar coronal loops", Astrophys. J. Lett., 232, L125-L129. [DOI], [ADS]. (Cited on page 35.)

Antiochos, S. K., 1984, "A dynamic model for the solar transition region", Astrophys. J., 280, 416-422. [DOI], $[\mathrm{ADS}]$. (Cited on page 46.)

Antiochos, S. K. and Klimchuk, J. A., 1991, "A model for the formation of solar prominences", Astrophys. J., 378, 372-377. [DOI], [ADS]. (Cited on page 53.)

Antiochos, S. K. and Noci, G., 1986, "The structure of the static corona and transition region", Astrophys. J., 301, 440-447. [DOI], [ADS]. (Cited on page 38.)

Antiochos, S. K., MacNeice, P. J., Spicer, D. S. and Klimchuk, J. A., 1999, "The Dynamic Formation of Prominence Condensations", Astrophys. J., 512, 985-991. [DOI], [ADS], [arXiv:astro-ph/9808199]. (Cited on pages 35 and 53.)

Antiochos, S. K., Karpen, J. T., DeLuca, E. E., Golub, L. and Hamilton, P., 2003, "Constraints on Active Region Coronal Heating", Astrophys. J., 590, 547-553. [DOI], [ADS]. (Cited on pages 27 and 52.)

Antolin, P. and Rouppe van der Voort, L., 2012, "Observing the Fine Structure of Loops through Highresolution Spectroscopic Observations of Coronal Rain with the CRISP Instrument at the Swedish Solar Telescope", Astrophys. J., 745, 152. [DOI], [ADS], [arXiv:1112.0656 [astro-ph.SR]]. (Cited on page 16.)

Antolin, P., Shibata, K., Kudoh, T., Shiota, D. and Brooks, D., 2008, "Predicting Observational Signatures of Coronal Heating by Alfvén Waves and Nanoflares", Astrophys. J., 688, 669-682. [DOI], [ADS]. (Cited on page 55.)

Antonucci, E., Gabriel, A. H., Acton, L. W. et al., 1982, "Impulsive phase of flares in soft X-ray emission", Solar Phys., 78, 107-123. [DOI], [ADS]. (Cited on page 32.)

Argiroffi, C., Peres, G., Orlando, S. and Reale, F., 2008, "The flaring and quiescent components of the solar corona", Astron. Astrophys., 488, 1069-1077. [DOI], [ADS], [arXiv:0805.2685]. (Cited on page 57.)

Aschwanden, M. J., 1999, "Do EUV Nanoflares Account for Coronal Heating?", Solar Phys., 190, 233-247. [DOI], [ADS]. (Cited on pages 51 and 52.)

Aschwanden, M. J., 2001, "Revisiting the Determination of the Coronal Heating Function from Yohkoh Data", Astrophys. J. Lett., 559, L171-L174. [DOI], [ADS]. (Cited on pages 45 and 50.)

Aschwanden, M. J., 2004, Physics of the Solar Corona: An Introduction, Springer-Praxis Books in Geophysical Sciences, Springer; Praxis, Berlin; New York; Chichester. [ADS], [Google Books]. (Cited on page 6.)

Aschwanden, M. J. and Nightingale, R. W., 2005, "Elementary Loop Structures in the Solar Corona Analyzed from TRACE Triple-Filter Images", Astrophys. J., 633, 499-517. [DOI], [ADS]. (Cited on pages 15,18 , and 26.)

Aschwanden, M. J. and Nitta, N., 2000, "The Effect of Hydrostatic Weighting on the Vertical Temperature Structure of the Solar Corona", Astrophys. J. Lett., 535, L59-L62. [DOI], [ADS], [arXiv:astroph/0004093]. (Cited on page 38.)

Aschwanden, M. J., Fletcher, L., Schrijver, C. J. and Alexander, D., 1999a, "Coronal Loop Oscillations Observed with the Transition Region and Coronal Explorer", Astrophys. J., 520, 880-894. [DOI], [ADS]. (Cited on pages 33 and 54.) 
Aschwanden, M. J., Newmark, J. S., Delaboudinière, J.-P., Neupert, W. M., Klimchuk, J. A., Gary, G. A., Portier-Fozzani, F. and Zucker, A., 1999b, "Three-dimensional stereoscopic analysis of solar active region loops. I. SOHO/EIT observations at temperatures of $(1.0-1.5) \times 10^{6} \mathrm{~K}$ ", Astrophys. J., 515, 842-867. [DOI], [ADS]. (Cited on page 25.)

Aschwanden, M. J., Nightingale, R. W. and Alexander, D., 2000, "Evidence for Nonuniform Heating of Coronal Loops Inferred from Multithread Modeling of TRACE Data", Astrophys. J., 541, 1059-1077. [DOI], [ADS]. (Cited on pages 11 and 25.)

Aschwanden, M. J., Poland, A. I. and Rabin, D. M., 2001, "The New Solar Corona", Annu. Rev. Astron. Astrophys., 39, 175-210. [DOI], [ADS]. (Cited on pages 6 and 18.)

Aschwanden, M. J., De Pontieu, B., Schrijver, C. J. and Title, A. M., 2002, "Transverse Oscillations in Coronal Loops Observed with TRACE II. Measurements of Geometric and Physical Parameters", Solar Phys., 206, 99-132. [DOI], [ADS]. (Cited on pages 13 and 22.)

Aschwanden, M. J., Nightingale, R. W. and Boerner, P., 2007, "A Statistical Model of the Inhomogeneous Corona Constrained by Triple-Filter Measurements of Elementary Loop Strands with TRACE", Astrophys. J., 656, 577-597. [DOI], [ADS]. (Cited on page 15.)

Aschwanden, M. J., Nitta, N. V., Wülser, J.-P. and Lemen, J. R., 2008, "First 3D Reconstructions of Coronal Loops with the STEREO A+B Spacecraft. II. Electron Density and Temperature Measurements", Astrophys. J., 680, 1477-1495. [DOI], [ADS]. (Cited on pages 14 and 18.)

Aschwanden, M. J., Wülser, J.-P., Nitta, N. V., Lemen, J. R. and Sandman, A., 2009, "First ThreeDimensional Reconstructions of Coronal Loops with the STEREO A+B Spacecraft. III. Instant Stereoscopic Tomography of Active Regions", Astrophys. J., 695, 12-29. [DOI], [ADS]. (Cited on pages 8, 14, and 25.)

Aschwanden, M. J., Wülser, J.-P., Nitta, N. V., Lemen, J. R., DeRosa, M. L. and Malanushenko, A., 2012, "First Three-dimensional Reconstructions of Coronal Loops with the STEREO A+B Spacecraft. IV. Magnetic Modeling with Twisted Force-free Fields", Astrophys. J., 756, 124. [DOI], [ADS], [arXiv:1207.2790 [astro-ph.SR]]. (Cited on page 14.)

Aschwanden, M. J., Boerner, P., Schrijver, C. J. and Malanushenko, A., 2013, "Automated Temperature and Emission Measure Analysis of Coronal Loops and Active Regions Observed with the Atmospheric Imaging Assembly on the Solar Dynamics Observatory (SDO/AIA)", Solar Phys., 283, 5-30. [DOI], [ADS]. (Cited on page 11.)

Asgari-Targhi, M., van Ballegooijen, A. A., Cranmer, S. R. and DeLuca, E. E., 2013, "The Spatial and Temporal Dependence of Coronal Heating by Alfvén Wave Turbulence", Astrophys. J., 773, 111. [DOI], [ADS], [arXiv:1306.6038 [astro-ph.SR]]. (Cited on page 55.)

Athay, R. G. and Holzer, T. E., 1982, "The role of spicules in heating the solar atmosphere", Astrophys. J., 255, 743-752. [DOI], [ADS]. (Cited on page 32.)

Bak, P., Chen, K. and Creutz, M., 1989, "Self-organized criticality in the 'Game of Life"', Nature, 342, 780-782. [DOI], [ADS]. (Cited on page 46.)

Ballai, I., Jess, D. B. and Douglas, M., 2011, "TRACE observations of driven loop oscillations", Astron. Astrophys., 534, A13. [DOI], [ADS], [arXiv:1108.2593 [astro-ph.SR]]. (Cited on page 12.)

Bareford, M. R., Browning, P. K. and van der Linden, R. A. M., 2010, "A nanoflare distribution generated by repeated relaxations triggered by kink instability", Astron. Astrophys., 521, A70. [DOI], [ADS], [arXiv:1005.5249 [astro-ph.SR]]. (Cited on pages 50 and 52.)

Bareford, M. R., Browning, P. K. and van der Linden, R. A. M., 2011, "The Flare-Energy Distributions Generated by Kink-Unstable Ensembles of Zero-Net-Current Coronal Loops", Solar Phys., 273, 93-115. [DOI], [ADS], [arXiv:1103.5378 [astro-ph.SR]]. (Cited on page 50.) 
Bareford, M. R., Hood, A. W. and Browning, P. K., 2013, "Coronal heating by the partial relaxation of twisted loops", Astron. Astrophys., 550, A40. [DOI], [ADS], [arXiv:1211.3855 [astro-ph.SR]]. (Cited on page 50.)

Bartoe, J.-D. F., Brueckner, G. E., Purcell, J. D. and Tousey, R., 1977, "Extreme ultraviolet spectrograph ATM experiment S082B", Appl. Opt., 16, 879-886. [ADS]. (Cited on page 7.)

Baty, H., 2000, "Magnetic reconnection in kinked coronal loops", Astron. Astrophys., 353, 1074-1082. [ADS]. (Cited on page 49.)

Beliën, A. J. C., Martens, P. C. H. and Keppens, R., 1999, "Coronal Heating by Resonant Absorption: The Effects of Chromospheric Coupling", Astrophys. J., 526, 478-493. [DOI], [ADS]. (Cited on page 54.)

Benz, A. O., 2008, "Flare Observations", Living Rev. Solar Phys., 5, lrsp-2008-1. [DOI], [ADS]. URL (accessed 26 October 2010): http://www.livingreviews.org/lrsp-2008-1. (Cited on page 26.)

Berger, T. E., De Pontieu, B., Fletcher, L., Schrijver, C. J., Tarbell, T. D. and Title, A. M., 1999, "What is Moss?", Solar Phys., 190, 409-418. [DOI], [ADS]. (Cited on pages 24 and 27.)

Berghmans, D. and Clette, F., 1999, "Active region EUV transient brightenings: First Results by EIT of SOHO JOP 80", Solar Phys., 186, 207-229. [DOI], [ADS]. (Cited on page 54.)

Berton, R. and Sakurai, T., 1985, "Stereoscopic determination of the three-dimensional geometry of coronal magnetic loops", Solar Phys., 96, 93-111. [DOI], [ADS]. (Cited on page 13.)

Betta, R., Peres, G., Reale, F. and Serio, S., 1997, "An adaptive grid code for high resolution 1-D hydrodynamics of the solar and stellar transition region and corona", Astron. Astrophys. Suppl., 122, 585-592. [DOI], [ADS]. (Cited on pages 35 and 36.)

Betta, R. M., Peres, G., Reale, F. and Serio, S., 2001, "Coronal loop hydrodynamics. The solar flare observed on November 12, 1980 revisited: The UV line emission", Astron. Astrophys., 380, 341-346. [DOI], [ADS], [arXiv:astro-ph/0110514]. (Cited on page 40.)

Beveridge, C., Longcope, D. W. and Priest, E. R., 2003, "A model for elemental coronal flux loops", Solar Phys., 216, 27-40. [DOI], [ADS]. (Cited on page 15.)

Bingert, S. and Peter, H., 2011, "Intermittent heating in the solar corona employing a 3D MHD model", Astron. Astrophys., 530, A112. [DOI], [ADS], [arXiv:1103.6042 [physics.space-ph]]. (Cited on page 56.)

Boerner, P., Edwards, C., Lemen, J. et al., 2012, "Initial Calibration of the Atmospheric Imaging Assembly (AIA) on the Solar Dynamics Observatory (SDO)", Solar Phys., 275, 41-66. [DOI], [ADS]. (Cited on page 8 .)

Bohlin, J. D., Frost, K. J., Burr, P. T., Guha, A. K. and Withbroe, G. L., 1980, "Solar Maximum Mission", Solar Phys., 65, 5-14. [DOI], [ADS]. (Cited on page 8.)

Borrini, G. and Noci, G., 1982, "Non-equilibrium ionization in coronal loops", Solar Phys., 77, 153-166. [DOI], [ADS]. (Cited on page 46.)

Botha, G. J. J., Arber, T. D., Nakariakov, V. M. and Keenan, F. P., 2000, “A developed stage of Alfvén wave phase mixing", Astron. Astrophys., 363, 1186-1194. [ADS]. (Cited on page 54.)

Bourouaine, S. and Marsch, E., 2010, "Multi-strand Coronal Loop Model and Filter-ratio Analysis", Astrophys. J., 708, 1281-1289. [DOI], [ADS], [arXiv:0911.3506 [astro-ph.SR]]. (Cited on pages 45 and 54.)

Boutry, C., Buchlin, E., Vial, J.-C. and Régnier, S., 2012, "Flows at the Edge of an Active Region: Observation and Interpretation", Astrophys. J., 752, 13. [DOI], [ADS], [arXiv:1204.1377 [astro-ph.SR]]. (Cited on page 29.) 
Bradshaw, S. J. and Cargill, P. J., 2005, "The cooling of coronal plasmas", Astron. Astrophys., 437, 311-317. [DOI], [ADS]. (Cited on page 48.)

Bradshaw, S. J. and Cargill, P. J., 2006, "Explosive heating of low-density coronal plasma", Astron. Astrophys., 458, 987-995. [DOI], [ADS]. (Cited on pages 35 and 36.)

Bradshaw, S. J. and Cargill, P. J., 2010, "The Cooling of Coronal Plasmas. III. Enthalpy Transfer as a Mechanism for Energy Loss", Astrophys. J., 717, 163-174. [DOI], [ADS]. (Cited on pages 41 and 48.)

Bradshaw, S. J. and Cargill, P. J., 2013, "The Influence of Numerical Resolution on Coronal Density in Hydrodynamic Models of Impulsive Heating", Astrophys. J., 770, 12. [DOI], [ADS], [arXiv:1305.1902 [astro-ph.SR]]. (Cited on pages 35 and 36.)

Bradshaw, S. J. and Klimchuk, J. A., 2011, "What Dominates the Coronal Emission Spectrum During the Cycle of Impulsive Heating and Cooling?", Astrophys. J. Suppl. Ser., 194, 26. [DOI], [ADS]. (Cited on page 52.)

Bradshaw, S. J. and Mason, H. E., 2003, "The radiative response of solar loop plasma subject to transient heating", Astron. Astrophys., 407, 1127-1138. [DOI], [ADS]. (Cited on page 35.)

Bradshaw, S. J., Klimchuk, J. A. and Reep, J. W., 2012, "Diagnosing the Time-dependence of Active Region Core Heating from the Emission Measure. I. Low-frequency Nanoflares", Astrophys. J., 758, 53. [DOI], [ADS], [arXiv:1209.0737 [astro-ph.SR]]. (Cited on page 53.)

Bray, R. J., Cram, L. E., Durrant, C. and Loughhead, R. E., 1991, Plasma loops in the solar corona, Cambridge Astrophysics Series, 18, Cambridge University Press, Cambridge. [ADS]. (Cited on pages 6 and 38.)

Brekke, P., 1993, "Observed redshifts in O V and downflows in the solar transition region", Astrophys. J., 408, 735-743. [DOI], [ADS]. (Cited on page 29.)

Brekke, P., 1999, "Observations of Transition Region Plasma", Solar Phys., 190, 379-408. [DOI], [ADS]. (Cited on page 31.)

Brekke, P., Kjeldseth-Moe, O. and Harrison, R. A., 1997, "High-Velocity Flows in an Active Region Loop System Observed with the Coronal Diagnostic Spectrometer (CDS) on SOHO", Solar Phys., 175, 511521. [DOI], [ADS]. (Cited on pages 11, 29, and 31.)

Brickhouse, N. S. and Schmelz, J. T., 2006, "The Transparency of Solar Coronal Active Regions", Astrophys. J. Lett., 636, L53-L56. [DOI], [ADS], [arXiv:astro-ph/0511683]. (Cited on page 24.)

Brković, A., Landi, E., Landini, M., Rüedi, I. and Solanki, S. K., 2002, "Models for solar magnetic loops. II. Comparison with SOHO-CDS observations on the solar disk", Astron. Astrophys., 383, 661-677. [DOI], [ADS]. (Cited on page 38.)

Brooks, D. H. and Warren, H. P., 2009, "Flows and Motions in Moss in the Core of a Flaring Active Region: Evidence for Steady Heating", Astrophys. J. Lett., 703, L10-L13. [DOI], [ADS], [arXiv:0905.3462 [astroph.SR]]. (Cited on page 31.)

Brooks, D. H. and Warren, H. P., 2012, "The Coronal Source of Extreme-ultraviolet Line Profile Asymmetries in Solar Active Region Outflows", Astrophys. J. Lett., 760, L5. [DOI], [ADS], [arXiv:1210.1274 [astro-ph.SR]]. (Cited on page 32.)

Brooks, D. H., Ugarte-Urra, I. and Warren, H. P., 2008, "The Role of Transient Brightenings in Heating the Solar Corona", Astrophys. J. Lett., 689, L77-L80. [DOI], [ADS]. (Cited on page 28.)

Brooks, D. H., Warren, H. P. and Young, P. R., 2011, "EUV Spectral Line Formation and the Temperature Structure of Active Region Fan Loops: Observations with Hinode/EIS and SDO/AIA", Astrophys. J., 730, 85. [DOI], [ADS], [arXiv:1101.5240 [astro-ph.SR]]. (Cited on page 26.) 
Brooks, D. H., Warren, H. P. and Ugarte-Urra, I., 2012, "Solar Coronal Loops Resolved by Hinode and the Solar Dynamics Observatory", Astrophys. J. Lett., 755, L33. [DOI], [ADS]. (Cited on page 16.)

Brooks, D. H., Warren, H. P., Ugarte-Urra, I. and Winebarger, A. R., 2013, "High Spatial Resolution Observations of Loops in the Solar Corona", Astrophys. J. Lett., 772, L19. [DOI], [ADS], [arXiv:1305.2246 [astro-ph.SR]]. (Cited on page 16.)

Brosius, J. W., Davila, J. M., Thomas, R. J. and Monsignori-Fossi, B. C., 1996, "Measuring Active and Quiet-Sun Coronal Plasma Properties with Extreme-Ultraviolet Spectra from SERTS", Astrophys. J. Suppl. Ser., 106, 143-164. [DOI], [ADS]. (Cited on pages 18 and 25.)

Browning, P. K., Gerrard, C., Hood, A. W., Kevis, R. and van der Linden, R. A. M., 2008, "Heating the corona by nanoflares: simulations of energy release triggered by a kink instability", Astron. Astrophys., 485, 837-848. [DOI], [ADS]. (Cited on page 56.)

Buchlin, E. and Velli, M., 2007, "Shell Models of RMHD Turbulence and the Heating of Solar Coronal Loops", Astrophys. J., 662, 701-714. [DOI], [ADS], [arXiv:astro-ph/0606610]. (Cited on page 56.)

Buchlin, E., Cargill, P. J., Bradshaw, S. J. and Velli, M., 2007, "Profiles of heating in turbulent coronal magnetic loops", Astron. Astrophys., 469, 347-354. [DOI], [ADS], [arXiv:astro-ph/0702748]. (Cited on page 56.)

Cargill, P. J., 1993, "The Fine Structure of a Nanoflare-Heated Corona", Solar Phys., 147, 263-268. [DOI], [ADS]. (Cited on page 51.)

Cargill, P. J., 1994, "Some implications of the nanoflare concept", Astrophys. J., 422, 381-393. [DOI], [ADS]. (Cited on pages 51 and 52.)

Cargill, P. J., 1995, "Diagnostics of Coronal Heating", in Infrared Tools for Solar Astrophysics: What's Next?, Proceedings of the Fifteenth NSO/Sac Peak Workshop, 19-23 September 1994, Sunspot, New Mexico, (Eds.) Kuhn, J. R., Penn, M. J., p. 17, World Scientific, Singapore. [ADS]. (Cited on page 6.)

Cargill, P. J. and Klimchuk, J. A., 1997, "A Nanoflare Explanation for the Heating of Coronal Loops Observed by YOHKOH", Astrophys. J., 478, 799. [DOI], [ADS]. (Cited on page 51.)

Cargill, P. J. and Klimchuk, J. A., 2004, "Nanoflare Heating of the Corona Revisited", Astrophys. J., 605, 911-920. [DOI], [ADS]. (Cited on pages 15, 41, 43, 51, and 52.)

Cargill, P. J. and Klimchuk, J. A., 2006, "On the Temperature-Emission Measure Distribution in Stellar Coronae", Astrophys. J., 643, 438-443. [DOI], [ADS]. (Cited on page 57.)

Cargill, P. J. and Priest, E. R., 1980, "Siphon flows in coronal loops: I. Adiabatic flow", Solar Phys., 65, 251-269. [DOI], [ADS]. (Cited on page 46.)

Cargill, P. J., Bradshaw, S. J. and Klimchuk, J. A., 2012a, "Enthalpy-based Thermal Evolution of Loops. II. Improvements to the Model", Astrophys. J., 752, 161. [DOI], [ADS], [arXiv:1204.5960 [astro-ph.SR]]. (Cited on page 37.)

Cargill, P. J., Bradshaw, S. J. and Klimchuk, J. A., 2012b, "Enthalpy-based Thermal Evolution of Loops. III. Comparison of Zero-dimensional Models", Astrophys. J., 758, 5. [DOI], [ADS]. (Cited on page 37.)

Cassak, P. A., Mullan, D. J. and Shay, M. A., 2008, "From Solar and Stellar Flares to Coronal Heating: Theory and Observations of How Magnetic Reconnection Regulates Coronal Conditions", Astrophys. J. Lett., 676, L69-L72. [DOI], [ADS], [arXiv:0710.3399]. (Cited on page 56.)

Chae, J., Schühle, U. and Lemaire, P., 1998a, "SUMER Measurements of Nonthermal Motions: Constraints on Coronal Heating Mechanisms", Astrophys. J., 505, 957-973. [DOI], [ADS]. (Cited on page 31.) 
Chae, J., Wang, H., Lee, C.-Y., Goode, P. R. and Schühle, U., 1998b, "Chromospheric Upflow Events Associated with Transition Region Explosive Events", Astrophys. J. Lett., 504, L123. [DOI], [ADS]. (Cited on page 30.)

Chae, J., Yun, H. S. and Poland, A. I., 1998c, "Temperature Dependence of Ultraviolet Line Average Doppler Shifts in the Quiet Sun", Astrophys. J. Suppl. Ser., 114, 151. [DOI], [ADS]. (Cited on pages 9 and 30.)

Charbonneau, P., McIntosh, S. W., Liu, H.-L. and Bogdan, T. J., 2001, "Avalanche models for solar flares (Invited Review)", Solar Phys., 203, 321-353. [DOI], [ADS]. (Cited on page 46.)

Chen, F. and Ding, M. D., 2010, "Evidence of Explosive Evaporation in a Microflare Observed by Hinode/EIS", Astrophys. J., 724, 640-648. [DOI], [ADS], [arXiv:1009.3193 [astro-ph.SR]]. (Cited on pages 29 and 32.)

Cheng, C.-C., Oran, E. S., Doschek, G. A., Boris, J. P. and Mariska, J. T., 1983, "Numerical simulations of loops heated to solar flare temperatures. I", Astrophys. J., 265, 1090-1119. [DOI], [ADS]. (Cited on page 40.)

Cirtain, J. W., Del Zanna, G., DeLuca, E. E., Mason, H. E., Martens, P. C. H. and Schmelz, J. T., 2007, "Active Region Loops: Temperature Measurements as a Function of Time from Joint TRACE and SOHO CDS Observations", Astrophys. J., 655, 598-605. [DOI], [ADS]. (Cited on page 28.)

Cirtain, J. W., Golub, L., Winebarger, A. R. et al., 2013, "Energy release in the solar corona from spatially resolved magnetic braids", Nature, 493, 501-503. [DOI], [ADS]. (Cited on pages 9 and 26.)

Craig, I. J. D., McClymont, A. N. and Underwood, J. H., 1978, "The Temperature and Density Structure of Active Region Coronal Loops", Astron. Astrophys., 70, 1. [ADS]. (Cited on page 38.)

Culhane, L., Harra, L. K., Baker, D. et al., 2007, "Hinode EUV Study of Jets in the Sun's South Polar Corona", Publ. Astron. Soc. Japan, 59, S751-S756. [ADS]. (Cited on page 8.)

Dadashi, N., Teriaca, L. and Solanki, S. K., 2011, "The quiet Sun average Doppler shift of coronal lines up to 2 MK", Astron. Astrophys., 534, A90. [DOI], [ADS], [arXiv:1109.4493 [astro-ph.SR]]. (Cited on page 30.)

Dadashi, N., Teriaca, L., Tripathi, D., Solanki, S. K. and Wiegelmann, T., 2012, "Doppler shift of hot coronal lines in a moss area of an active region", Astron. Astrophys., 548, A115. [DOI], [ADS], [arXiv:1211.5473 [astro-ph.SR]]. (Cited on pages 30 and 31.)

Dahlburg, R. B., Einaudi, G., Rappazzo, A. F. and Velli, M., 2012, "Turbulent coronal heating mechanisms: coupling of dynamics and thermodynamics", Astron. Astrophys., 544, L20. [DOI], [ADS], [arXiv:1208.2459 [astro-ph.SR]]. (Cited on page 45.)

Davila, J. M., 1987, "Heating of the solar corona by the resonant absorption of Alfvén waves", Astrophys. J., 317, 514-521. [DOI], [ADS]. (Cited on page 53.)

De Groof, A., Berghmans, D., van Driel-Gesztelyi, L. and Poedts, S., 2004, "Intensity variations in EIT shutterless mode: Waves or flows?", Astron. Astrophys., 415, 1141-1151. [DOI], [ADS]. (Cited on pages 33 and 48.)

De Moortel, I. and Nakariakov, V. M., 2012, "Magnetohydrodynamic waves and coronal seismology: an overview of recent results", Philos. Trans. R. Soc. London, Ser. A, 370, 3193-3216. [DOI], [ADS], [arXiv:1202.1944 [astro-ph.SR]]. (Cited on page 6.)

De Moortel, I., Ireland, J. and Walsh, R. W., 2000, "Observation of oscillations in coronal loops", Astron. Astrophys., 355, L23-L26. [ADS]. (Cited on page 33.) 
De Moortel, I., Ireland, J., Walsh, R. W. and Hood, A. W., 2002, "Longitudinal intensity oscillations in coronal loops observed with TRACE I. Overview of Measured Parameters", Solar Phys., 209, 61-88. [DOI], $[\mathrm{ADS}]$. (Cited on page 54.)

De Pontieu, B. and McIntosh, S. W., 2010, "Quasi-periodic Propagating Signals in the Solar Corona: The Signature of Magnetoacoustic Waves or High-velocity Upflows?", Astrophys. J., 722, 1013-1029. [DOI], [ADS], [arXiv:1008.5300 [astro-ph.SR]]. (Cited on page 33.)

De Pontieu, B., McIntosh, S., Hansteen, V. H. et al., 2007a, "A Tale of Two Spicules: The Impact of Spicules on the Magnetic Chromosphere", Publ. Astron. Soc. Japan, 59, S655-S662. [DOI], [ADS], [arXiv:0710.2934]. (Cited on page 32.)

De Pontieu, B., McIntosh, S. W., Carlsson, M. et al., 2007b, "Chromospheric Alfvénic Waves Strong Enough to Power the Solar Wind", Science, 318, 1574-1577. [DOI], [ADS]. (Cited on page 54.)

De Pontieu, B., McIntosh, S. W., Hansteen, V. H. and Schrijver, C. J., 2009, "Observing the roots of solar coronal heating - in the chromosphere", Astrophys. J. Lett., 701, L1-L6. [DOI], [ADS], [arXiv:0906.5434]. (Cited on page 32.)

De Pontieu, B., McIntosh, S. W., Carlsson, M. et al., 2011, "The Origins of Hot Plasma in the Solar Corona", Science, 331, 55. [DOI], [ADS]. (Cited on pages 26 and 32.)

DeForest, C. E., 2007, "On the Size of Structures in the Solar Corona", Astrophys. J., 661, 532-542. [DOI], [ADS], [arXiv:astro-ph/0610178]. (Cited on page 15.)

Del Zanna, G., 2008, "Flows in active region loops observed by Hinode EIS", Astron. Astrophys., 481, L49-L52. [DOI], [ADS]. (Cited on page 30.)

Del Zanna, G., 2012, "Benchmarking atomic data for astrophysics: a first look at the soft X-ray lines", Astron. Astrophys., 546, A97. [DOI], [ADS], [arXiv:1208.2142 [astro-ph.SR]]. (Cited on page 18.)

Del Zanna, G. and Mason, H. E., 2003, "Solar active regions: SOHO/CDS and TRACE observations of quiescent coronal loops", Astron. Astrophys., 406, 1089-1103. [DOI], [ADS]. (Cited on pages 18, 22, and 25.)

Del Zanna, G., O’Dwyer, B. and Mason, H. E., 2011, "SDO AIA and Hinode EIS observations of "warm" loops", Astron. Astrophys., 535, A46. [DOI], [ADS]. (Cited on page 26.)

Delaboudinière, J.-P., Artzner, G. E., Brunaud, J. et al., 1995, "EIT: Extreme-Ultraviolet Imaging Telescope for the SOHO Mission", Solar Phys., 162, 291-312. [DOI], [ADS]. (Cited on page 8.)

Démoulin, P., van Driel-Gesztelyi, L., Mandrini, C. H., Klimchuk, J. A. and Harra, L., 2003, "The LongTerm Evolution of AR 7978: Testing Coronal Heating Models", Astrophys. J., 586, 592-605. [DOI], [ADS]. (Cited on page 55.)

Dere, K. P., 1982, "Extreme ultraviolet spectra of solar active regions and their analysis", Solar Phys., 77, 77-93. [DOI], [ADS]. (Cited on page 29.)

Dere, K. P., 2008, "The plasma filling factor of coronal bright points. Coronal bright points", Astron. Astrophys., 491, 561-566. [DOI], [ADS]. (Cited on page 21.)

Dere, K. P., 2009, "The plasma filling factor of coronal bright points. II. Combined EIS and TRACE results", Astron. Astrophys., 497, 287-290. [DOI], [ADS]. (Cited on page 21.)

Dere, K. P., Bartoe, J.-D. F. and Brueckner, G. E., 1986, "Outflows and ejections in the solar transition zone", Astrophys. J., 310, 456-462. [DOI], [ADS]. (Cited on page 29.)

Dere, K. P., Bartoe, J.-D. F. and Brueckner, G. E., 1989, "Explosive events in the solar transition zone", Solar Phys., 123, 41-68. [DOI], [ADS]. (Cited on page 30.) 
Dere, K. P., Landi, E., Mason, H. E., Monsignori Fossi, B. C. and Young, P. R., 1997, "CHIANTI - an atomic database for emission lines", Astron. Astrophys. Suppl., 125, 149-173. [DOI], [ADS]. (Cited on page 18.)

Dere, K. P., Landi, E., Young, P. R. and Del Zanna, G., 2001, "CHIANTI - An Atomic Database for Emission Lines. IV. Extension to X-Ray Wavelengths", Astrophys. J. Suppl. Ser., 134, 331-354. [DOI], [ADS]. (Cited on page 18.)

Dere, K. P., Landi, E., Young, P. R., Del Zanna, G., Landini, M. and Mason, H. E., 2009, "CHIANTI - an atomic database for emission lines. IX. Ionization rates, recombination rates, ionization equilibria for the elements hydrogen through zinc and updated atomic data", Astron. Astrophys., 498, 915-929. [DOI], [ADS]. (Cited on page 18.)

DeRosa, M. L., Schrijver, C. J., Barnes, G. et al., 2009, "A Critical Assessment of Nonlinear Force-Free Field Modeling of the Solar Corona for Active Region 10953", Astrophys. J., 696, 1780-1791. [DOI], [ADS], [arXiv:0902.1007 [astro-ph.SR]]. (Cited on page 14.)

Di Giorgio, S., Reale, F. and Peres, G., 2003, "CDS/SoHO multi-line observation of a solar active region: Detection of a hot stable loop and of a cool dynamic loop", Astron. Astrophys., 406, 323-335. [DOI], [ADS]. (Cited on pages 22, 23, 26, 27, 29, and 31.)

Di Matteo, V., Reale, F., Peres, G. and Golub, L., 1999, "Analysis and comparison of loop structures imaged with NIXT and Yohkoh/SXT", Astron. Astrophys., 342, 563-574. [ADS]. (Cited on pages 15 and 21.)

Domingo, V., Fleck, B. and Poland, A. I., 1995, "The SOHO Mission: an Overview", Solar Phys., 162, 1-37. [DOI], [ADS]. (Cited on page 8.)

Doschek, G. A., Bohlin, J. D. and Feldman, U., 1976, "Doppler wavelength shifts of transition zone lines measured in SKYLAB solar spectra", Astrophys. J. Lett., 205, L177-L180. [DOI], [ADS]. (Cited on page 29.)

Doschek, G. A., Boris, J. P., Cheng, C. C., Mariska, J. T. and Oran, E. S., 1982, "A Numerical Simulation of Cooling Coronal Flare Plasma", Astrophys. J., 258, 373. [DOI], [ADS]. (Cited on page 35.)

Doschek, G. A., Mariska, J. T., Warren, H. P., Culhane, L., Watanabe, T., Young, P. R., Mason, H. E. and Dere, K. P., 2007, "The Temperature and Density Structure of an Active Region Observed with the Extreme-Ultraviolet Imaging Spectrometer on Hinode", Publ. Astron. Soc. Japan, 59, 707. [ADS]. (Cited on pages 20 and 31.)

Doschek, G. A., Warren, H. P., Mariska, J. T., Muglach, K., Culhane, J. L., Hara, H. and Watanabe, T., 2008, "Flows and Nonthermal Velocities in Solar Active Regions Observed with the EUV Imaging Spectrometer on Hinode: A Tracer of Active Region Sources of Heliospheric Magnetic Fields?", Astrophys. J., 686, 1362-1371. [DOI], [ADS], [arXiv:0807.2860]. (Cited on page 31.)

Dudok de Wit, T. and Auchère, F., 2007, "Multispectral analysis of solar EUV images: linking temperature to morphology", Astron. Astrophys., 466, 347-355. [DOI], [ADS], [arXiv:astro-ph/0702052]. (Cited on page 15.)

Dudok de Wit, T., Moussaoui, S., Guennou, C., Auchère, F., Cessateur, G., Kretzschmar, M., Vieira, L. A. and Goryaev, F. F., 2013, "Coronal Temperature Maps from Solar EUV Images: A Blind Source Separation Approach", Solar Phys., 283, 31-47. [DOI], [ADS], [arXiv:1203.0116 [astro-ph.SR]]. (Cited on page 17.)

Dymova, M. V. and Ruderman, M. S., 2006, "The geometry effect on transverse oscillations of coronal loops", Astron. Astrophys., 459, 241-244. [DOI], [ADS]. (Cited on page 13.)

Edlén, B., 1943, "Die Deutung der Emissionslinien im Spektrum der Sonnenkorona", Z. Astrophys., 22, 30-64. [ADS]. (Cited on page 5.) 
Favata, F., Reale, F., Micela, G., Sciortino, S., Maggio, A. and Matsumoto, H., 2000, "An extreme X-ray flare observed on EV Lac by ASCA in July 1998", Astron. Astrophys., 353, 987-997. [ADS], [arXiv:astro-ph/9909491]. (Cited on page 42.)

Favata, F., Flaccomio, E., Reale, F., Micela, G., Sciortino, S., Shang, H., Stassun, K. G. and Feigelson, E. D., 2005, "Bright X-Ray Flares in Orion Young Stars from COUP: Evidence for Star-Disk Magnetic Fields?", Astrophys. J. Suppl. Ser., 160, 469-502. [DOI], [ADS], [arXiv:astro-ph/0506134]. (Cited on page 57.)

Feldman, U., Doschek, G. A. and Mariska, J. T., 1979, "On the structure of the solar transition zone and lower corona", Astrophys. J., 229, 369-374. [DOI], [ADS]. (Cited on page 18.)

Feldman, U., Doschek, G. A. and Cohen, L., 1982, "Doppler wavelength shifts of ultraviolet spectral lines in solar active regions", Astrophys. J., 255, 325-328. [DOI], [ADS]. (Cited on page 29.)

Feldman, U., Dammasch, I. E. and Doschek, G. A., 2011, "Redshifts, Widths, and Radiances of Spectral Lines Emitted by the Solar Transition Region", Astrophys. J., 743, 165. [DOI], [ADS]. (Cited on page 29.)

Feng, L., Inhester, B., Solanki, S. K., Wiegelmann, T., Podlipnik, B., Howard, R. A. and Wülser, J.-P., 2007, "First stereoscopic coronal loop reconstructions from STEREO SECCHI images", Astrophys. J. Lett., 671, L205-L208. [DOI], [ADS], [arXiv:0802.0773]. (Cited on page 13.)

Fisher, G. H., Canfield, R. C. and McClymont, A. N., 1985a, "Flare loop radiative hydrodynamics. VII. Dynamics of the thick-target heated chromosphere", Astrophys. J., 289, 434-441. [DOI], [ADS]. (Cited on pages 35 and 40.)

Fisher, G. H., Canfield, R. C. and McClymont, A. N., 1985b, "Flare loop radiative hydrodynamics. VI. Chromospheric evaporation due to heating by nonthermal electrons", Astrophys. J., 289, 425-433. [DOI], [ADS]. (Cited on page 40.)

Fisher, G. H., Canfield, R. C. and McClymont, A. N., 1985c, "Flare loop radiative hydrodynamics. V. Response to thick-target heating", Astrophys. J., 289, 414-424. [DOI], [ADS]. (Cited on page 40.)

Fletcher, L. and De Pontieu, B., 1999, "Plasma Diagnostics of Transition Region 'Moss' using SOHO/CDS and TRACE", Astrophys. J. Lett., 520, L135-L138. [DOI], [ADS]. (Cited on page 24.)

Foukal, P., 1975, "The temperature structure and pressure balance of magnetic loops in active regions", Solar Phys., 43, 327-336. [DOI], [ADS]. (Cited on page 7.)

Foukal, P. V., 1976, "The pressure and energy balance of the cool corona over sunspots", Astrophys. J., 210, 575-581. [DOI], [ADS]. (Cited on pages 7, 11, 27, and 29.)

Fuentes-Fernández, J., Parnell, C. E., Hood, A. W., Priest, E. R. and Longcope, D. W., 2012, "Consequences of spontaneous reconnection at a two-dimensional non-force-free current layer", Phys. Plasmas, 19(2), 022901. [DOI], [ADS], [arXiv:1202.0161 [astro-ph.SR]]. (Cited on page 50.)

Gabriel, A. H., 1976, "A magnetic model of the solar transition region", Philos. Trans. R. Soc. London, Ser. A, 281, 339-352. [ADS]. (Cited on pages 9 and 37.)

Gabriel, A. H. and Jordan, C., 1975, "Analysis of EUV observations of regions of the quiet and active corona at the time of the 1970 March 7 eclipse", Mon. Not. R. Astron. Soc., 173, 397-418. [ADS]. (Cited on page 18.)

Galeev, A. A., Rosner, R., Serio, S. and Vaiana, G. S., 1981, "Dynamics of coronal structures: Magnetic field-related heating and loop energy balance", Astrophys. J., 243, 301-308. [DOI], [ADS]. (Cited on page 49.)

Gan, W. Q., Zhang, H. Q. and Fang, C., 1991, "A hydrodynamic model of the impulsive phase of a solar flare loop", Astron. Astrophys., 241, 618-624. [ADS]. (Cited on page 35.) 
Gburek, S., Sylwester, J., Kowalinski, M. et al., 2013, "SphinX: The Solar Photometer in X-Rays", Solar Phys., 283, 631-649. [DOI], [ADS]. (Cited on page 8.)

Gebbie, K. B., Hill, F., November, L. J. et al., 1981, "Steady flows in the solar transition region observed with SMM", Astrophys. J. Lett., 251, L115-L118. [DOI], [ADS]. (Cited on page 29.)

Getman, K. V., Feigelson, E. D., Micela, G., Jardine, M. M., Gregory, S. G. and Garmire, G. P., 2008, "X-Ray Flares in Orion Young Stars. II. Flares, Magnetospheres, and Protoplanetary Disks", Astrophys. J., 688, 437-455. [DOI], [ADS], [arXiv:0807.3007]. (Cited on page 57.)

Giacconi, R., Reidy, W. P., Zehnpfennig, T., Lindsay, J. C. and Muney, W. S., 1965, "Solar X-Ray Image Obtained Using Grazing-Incidence Optics", Astrophys. J., 142, 1274-1278. [DOI], [ADS]. (Cited on page 7.)

Golub, L., 1996, "The Solar X-Ray Corona", Astrophys. J. Suppl. Ser., 237, 33-48. [DOI], [ADS]. (Cited on page 6.)

Golub, L. and Herant, M., 1989, "Analysis of the 23 June 1988 flare using NIXT multilayer X-ray images", in X-Ray/EUV Optics for Astronomy and Microscopy, San Diego, CA, August 7-11, 1989, (Ed.) Hoover, R. B., Proc. SPIE, 1160, pp. 629-635, SPIE, Bellingham, WA. [ADS]. (Cited on pages 8 and 46.)

Golub, L. and Pasachoff, J. M., 1997, The Solar Corona, Cambridge University Press, Cambridge. [ADS], [Google Books]. (Cited on page 6.)

Golub, L. and Pasachoff, J. M., 2001, Nearest Star: The Surprising Science of Our Sun, Harvard University Press, Cambridge, MA. [ADS]. (Cited on page 6.)

Golub, L., Maxson, C., Rosner, R., Vaiana, G. S. and Serio, S., 1980, "Magnetic fields and coronal heating", Astrophys. J., 238, 343-348. [DOI], [ADS]. (Cited on page 49.)

Golub, L., Noci, G., Poletto, G. and Vaiana, G. S., 1982, "Active region coronal evolution", Astrophys. J., 259, 359-365. [DOI], [ADS]. (Cited on pages 7 and 49.)

Golub, L., DeLuca, E. E., Austin, G. et al., 2007, "The X-Ray Telescope (XRT) for the Hinode Mission", Solar Phys., 243, 63-86. [DOI], [ADS]. (Cited on page 8.)

Gómez, D. O., Martens, P. C. H. and Golub, L., 1993, "Normal incidence X-ray telescope power spectra of X-ray emission from solar active regions. I. Observations. II. Theory", Astrophys. J., 405, 767-781. [DOI], [ADS]. (Cited on page 15.)

Gontikakis, C., Contopoulos, I. and Dara, H. C., 2008, "Distribution of coronal heating in a solar active region", Astron. Astrophys., 489, 441-447. [DOI], [ADS]. (Cited on page 38.)

Grappin, R., Léorat, J. and Ofman, L., 2003, "Flows in coronal loops driven by Alfvén waves: 1.5 MHD simulations with transparent boundary conditions", in Solar Wind Ten, Proceedings of the Tenth International Solar Wind Conference, Pisa, Italy, 17-21 June 2002, (Eds.) Velli, M., Bruno, R., Malara, F., AIP Conference Proceedings, 679, pp. 750-753, American Institute of Physics, Melville, NY. [DOI], [ADS]. (Cited on page 48.)

Grappin, R., Léorat, J. and Habbal, S. R., 2005, "Siphon flows and oscillations in long coronal loops due to Alfvén waves", Astron. Astrophys., 437, 1081-1092. [DOI], [ADS]. (Cited on page 48.)

Griffiths, N. W., Fisher, G. H., Woods, D. T., Acton, L. W. and Siegmund, O. H. W., 2000, "Simultaneous SOHO and Yohkoh Observations of a Small Solar Active Region", Astrophys. J., 537, 481-494. [DOI], [ADS]. (Cited on page 21.)

Grotrian, W., 1939, "Zur Frage der Deutung der Linien im Spektrum der Sonnenkorona", Naturwissenschaften, 27, 214. [DOI], [ADS]. (Cited on page 5.) 
Guarrasi, M., Reale, F. and Peres, G., 2010, "Coronal Fuzziness Modeled with Pulse-heated Multi-stranded Loop Systems", Astrophys. J., 719, 576-582. [DOI], [ADS]. (Cited on pages 16, 21, 24, 37, 44, and 45.)

Güdel, M., 2004, "X-ray astronomy of stellar coronae", Astron. Astrophys. Rev., 12, 71-237. [DOI], [ADS], [arXiv:astro-ph/0406661]. (Cited on page 57.)

Gudiksen, B. V. and Nordlund, A., 2005, "An Ab Initio Approach to the Solar Coronal Heating Problem", Astrophys. J., 618, 1020-1030. [DOI], [ADS], [arXiv:astro-ph/0407266]. (Cited on pages 37, 49, and 55.)

Gudiksen, B. V., Carlsson, M., Hansteen, V. H., Hayek, W., Leenaarts, J. and Martínez-Sykora, J., 2011, "The stellar atmosphere simulation code Bifrost: Code description and validation", Astron. Astrophys., 531, A154. [DOI], [ADS], [1105.6306 [astro-ph.SR]]. (Cited on page 55.)

Guennou, C., Auchère, F., Soubrié, E., Bocchialini, K., Parenti, S. and Barbey, N., 2012a, "On the Accuracy of the Differential Emission Measure Diagnostics of Solar Plasmas. Application to SDO/AIA. I. Isothermal Plasmas", Astrophys. J. Suppl. Ser., 203, 25. [DOI], [ADS], [arXiv:1210.2304 [astro-ph.SR]]. (Cited on page 18.)

Guennou, C., Auchère, F., Soubrié, E., Bocchialini, K., Parenti, S. and Barbey, N., 2012b, "On the Accuracy of the Differential Emission Measure Diagnostics of Solar Plasmas. Application to SDO/AIA. II. Multithermal Plasmas", Astrophys. J. Suppl. Ser., 203, 26. [DOI], [ADS], [arXiv:1210.2302 [astroph.SR]]. (Cited on page 18.)

Guerreiro, N., Hansteen, V. and De Pontieu, B., 2013, "The Cycling of Material between the Solar Corona and Chromosphere", Astrophys. J., 769, 47. [DOI], [ADS]. (Cited on page 48.)

Habbal, S. R., Ronan, R. and Withbroe, G. L., 1985, "Spatial and temporal variations of solar coronal loops", Solar Phys., 98, 323-340. [DOI], [ADS]. (Cited on page 27.)

Haisch, B. M., Strong, K. T., Harrison, R. A. and Gary, G. A., 1988, "Active region coronal loops: Structure and variability", Astrophys. J. Suppl. Ser., 68, 371-405. [DOI], [ADS]. (Cited on page 27.)

Handy, B. N., Acton, L. W., Kankelborg, C. C. et al., 1999, "The transition region and coronal explorer", Solar Phys., 187, 229-260. [DOI], [ADS]. (Cited on page 8.)

Hannah, I. G. and Kontar, E. P., 2012, "Differential emission measures from the regularized inversion of Hinode and SDO data", Astron. Astrophys., 539, A146. [DOI], [ADS], [arXiv:1201.2642 [astro-ph.SR]]. (Cited on page 18.)

Hansteen, V., 1993, "A new interpretation of the redshift observed in optically thin transition region lines", Astrophys. J., 402, 741-755. [DOI], [ADS]. (Cited on pages 29, 35, and 46.)

Hansteen, V. H., Carlsson, M. and Gudiksen, B., 2007, "3D Numerical Models of the Chromosphere, Transition Region, and Corona", in The Physics of Chromospheric Plasmas, Coimbra, Portugal, 9-13 October 2006, (Eds.) Heinzel, P., Dorotovič, I., Rutten, R. J., ASP Conference Series, 368, pp. 107114, Astronomical Society of the Pacific, San Francisco. [ADS], [arXiv:0704.1511 [astro-ph]]. (Cited on page 37.)

Hansteen, V. H., Hara, H., De Pontieu, B. and Carlsson, M., 2010, "On Redshifts and Blueshifts in the Transition Region and Corona", Astrophys. J., 718, 1070-1078. [DOI], [ADS], [arXiv:1001.4769 [astroph.SR]]. (Cited on pages 48 and 49.)

Hara, H., Tsuneta, S., Lemen, J. R., Acton, L. W. and McTiernan, J. M., 1992, "High-temperature plasmas in active regions observed with the Soft X-ray Telescope aboard YOHKOH", Publ. Astron. Soc. Japan, 44, L135-L140. [ADS]. (Cited on page 8.)

Hara, H., Watanabe, T., Harra, L. K., Culhane, J. L., Young, P. R., Mariska, J. T. and Doschek, G. A., 2008, "Coronal Plasma Motions near Footpoints of Active Region Loops Revealed from Spectroscopic Observations with Hinode EIS", Astrophys. J. Lett., 678, L67-L71. [DOI], [ADS]. (Cited on page 31.) 
Harrison, R. A., Sawyer, E. C., Carter, M. K. et al., 1995, "The Coronal Diagnostic Spectrometer for the Solar and Heliospheric Observatory", Solar Phys., 162, 233-290. [DOI], [ADS]. (Cited on page 8.)

Haynes, M., Arber, T. D. and Verwichte, E., 2008, "Coronal loop slow mode oscillations driven by the kink instability", Astron. Astrophys., 479, 235-239. [DOI], [ADS]. (Cited on page 56.)

Heyvaerts, J. and Priest, E. R., 1983, "Coronal heating by phase-mixed shear Alfvén waves", Astron. Astrophys., 117, 220-234. [ADS]. (Cited on page 54.)

Hollweg, J. V., 1981, "Alfvén waves in the solar atmosphere II: Open and closed magnetic flux tubes", Solar Phys., 70, 25-66. [DOI], [ADS]. (Cited on page 53.)

Hollweg, J. V., 1984, "Resonances of coronal loops", Astrophys. J., 277, 392-403. [DOI], [ADS]. (Cited on page 53.)

Hollweg, J. V., 1985, "Viscosity in a Magnetized Plasma: Physical Interpretation", J. Geophys. Res., 90, 7620-7622. [DOI], [ADS]. (Cited on page 53.)

Hollweg, J. V., 1986, "Transition region, corona, and solar wind in coronal holes", J. Geophys. Res., 91, 4111-4125. [DOI], [ADS]. (Cited on page 54.)

Hollweg, J. V. and Yang, G., 1988, "Resonance Absorption of Compressible Magnetohydrodynamic Waves at Thin 'Surfaces"', J. Geophys. Res., 93, 5423-5436. [DOI], [ADS]. (Cited on page 54.)

Hood, A. W. and Priest, E. R., 1979a, "The equilibrium of solar coronal magnetic loops", Astron. Astrophys., 77, 233-251. [ADS]. (Cited on page 38.)

Hood, A. W. and Priest, E. R., 1979b, "Kink instability of solar coronal loops as the cause of solar flares", Solar Phys., 64, 303-321. [DOI], [ADS]. (Cited on page 49.)

Hood, A. W. and Priest, E. R., 1980, "Are solar coronal loops in thermal equilibrium", Astron. Astrophys., 87, 126-131. [ADS]. (Cited on page 35.)

Hood, A. W., Archontis, V., Galsgaard, K. and Moreno-Insertis, F., 2009a, "The emergence of toroidal flux tubes from beneath the solar photosphere", Astron. Astrophys., 503, 999-1011. [DOI], [ADS]. (Cited on page 50.)

Hood, A. W., Browning, P. K. and van der Linden, R. A. M., 2009b, "Coronal heating by magnetic reconnection in loops with zero net current", Astron. Astrophys., 506, 913-925. [DOI], [ADS]. (Cited on page 50.)

Hori, K., Yokoyama, T., Kosugi, T. and Shibata, K., 1997, "Pseudo-Two-dimensional Hydrodynamic Modeling of Solar Flare Loops", Astrophys. J., 489, 426-441. [DOI], [ADS]. (Cited on page 40.)

Hori, K., Yokoyama, T., Kosugi, T. and Shibata, K., 1998, "Single and Multiple Solar Flare Loops: Hydrodynamics and Ca XIX Resonance Line Emission", Astrophys. J., 500, 492-510. [DOI], [ADS]. (Cited on page 40.)

Huang, Z., Frazin, R. A., Landi, E., Manchester, W. B., Vásquez, A. M. and Gombosi, T. I., 2012, "Newly Discovered Global Temperature Structures in the Quiet Sun at Solar Minimum", Astrophys. J., 755, 86. [DOI], [ADS], [arXiv:1207.6661 [astro-ph.SR]]. (Cited on page 11.)

Hudson, H. S., 1991, "Solar flares, microflares, nanoflares, and coronal heating", Solar Phys., 133, 357-369. [DOI], [ADS]. (Cited on page 52.)

Ignatiev, A. P., Kolachevsky, N. N., Korneev, V. V. et al., 1998, "Manufacture and testing of x-ray optical elements for the TEREK-C and RES-C instruments on the CORONAS-I mission", in Current Russian Research in Optics and Photonics: New Methods and Instruments for Space- and Earth-based Spectroscopy in XUV, UV, IR, and Millimeter Waves, (Eds.) Sobelman, I. I., Slemzin, V. A., Proc. SPIE, 3406, pp. 20-34, SPIE, Bellingham, WA. [DOI], [ADS]. (Cited on page 8.) 
Imada, S., Murakami, I., Watanabe, T., Hara, H. and Shimizu, T., 2011, "Magnetic Reconnection in Nonequilibrium Ionization Plasma", Astrophys. J., 742, 70. [DOI], [ADS], [arXiv:1108.5026 [astro-ph.SR]]. (Cited on page 46.)

Ionson, J. A., 1978, "Resonant absorption of Alfvénic surface waves and the heating of solar coronal loops", Astrophys. J., 226, 650-673. [DOI], [ADS]. (Cited on page 53.)

Ionson, J. A., 1982, "Resonant electrodynamic heating of stellar coronal loops: an LRC circuit analog", Astrophys. J., 254, 318-334. [DOI], [ADS]. (Cited on page 53.)

Ionson, J. A., 1983, "Electrodynamic coupling in magnetically confined X-ray plasmas of astrophysical origin", Astrophys. J., 271, 778-792. [DOI], [ADS]. (Cited on page 53.)

Jakimiec, J., Sylwester, B., Sylwester, J., Serio, S., Peres, G. and Reale, F., 1992, "Dynamics of flaring loops II. Flare evolution in the density-temperature diagram", Astron. Astrophys., 253, 269-276. [ADS]. (Cited on pages 40, 42, and 43.)

Ji, H., Cao, W. and Goode, P. R., 2012, "Observation of Ultrafine Channels of Solar Corona Heating", Astrophys. J. Lett., 750, L25. [DOI], [ADS]. (Cited on page 16.)

Jordan, C., 1976, "The structure and energy balance of solar active regions", Philos. Trans. R. Soc. London, Ser. A, 281, 391-404. [ADS]. (Cited on page 37.)

Jordan, C., 1980, "The energy balance of the solar transition region", Astron. Astrophys., 86, 355-363. [ADS]. (Cited on pages 37 and 51.)

Jordan, C., Ayres, T. R., Brown, A., Linsky, J. L. and Simon, T., 1987, "The chromospheres and coronae of five G-K main-sequence stars", Mon. Not. R. Astron. Soc., 225, 903-937. [ADS]. (Cited on page 18.)

Judge, P. G., Hansteen, V., Wikstol, O., Wilhelm, K., Schühle, U. and Moran, T., 1998, "Evidence in Support of the 'Nanoflare' Picture of Coronal Heating from SUMER Data", Astrophys. J., 502, 981. [DOI], [ADS]. (Cited on page 51.)

Judge, P. G., De Pontieu, B., McIntosh, S. W. and Olluri, K., 2012, "The Connection of Type II Spicules to the Corona", Astrophys. J., 746, 158. [DOI], [ADS], [arXiv:1112.6174 [astro-ph.SR]]. (Cited on page 48.)

Kaiser, M. L., Kucera, T. A., Davila, J. M., St Cyr, O. C., Guhathakurta, M. and Christian, E., 2008, "The STEREO mission: An introduction", Space Sci. Rev., 136, 5-16. [DOI], [ADS]. (Cited on page 8.)

Kamio, S., Peter, H., Curdt, W. and Solanki, S. K., 2011, "Continuous upflows and sporadic downflows observed in active regions", Astron. Astrophys., 532, A96. [DOI], [ADS], [arXiv:1107.1993 [astro-ph.SR]]. (Cited on page 32.)

Kano, R. and Tsuneta, S., 1995, "Scaling Law of Solar Coronal Loops Obtained with YOHKOH", Astrophys. J., 454, 934. [DOI], [ADS]. (Cited on pages 20 and 38.)

Karpen, J. T. and Antiochos, S. K., 2008, "Condensation Formation by Impulsive Heating in Prominences", Astrophys. J., 676, 658-671. [DOI], [ADS]. (Cited on page 53.)

Kashyap, V. and Drake, J. J., 1998, "Markov-Chain Monte Carlo Reconstruction of Emission Measure Distributions: Application to Solar Extreme-Ultraviolet Spectra", Astrophys. J., 503, 450. [DOI], [ADS]. (Cited on page 18.)

Katsukawa, Y. and Tsuneta, S., 2001, "Small Fluctuation of Coronal X-Ray Intensity and a Signature of Nanoflares", Astrophys. J., 557, 343-350. [DOI], [ADS]. (Cited on pages 51 and 52.)

Katsukawa, Y. and Tsuneta, S., 2005, "Magnetic Properties at Footpoints of Hot and Cool Loops", Astrophys. J., 621, 498-511. [DOI], [ADS]. (Cited on page 15.) 
Klimchuk, J. A., 1987, "On the large-scale dynamics and magnetic structure of solar active regions", Astrophys. J., 323, 368-379. [DOI], [ADS]. (Cited on page 29.)

Klimchuk, J. A., 2000, "Cross-Sectional Properties of Coronal Loops", Solar Phys., 193, 53-75. [ADS]. (Cited on pages 14 and 49.)

Klimchuk, J. A., 2006, "On Solving the Coronal Heating Problem", Solar Phys., 234, 41-77. [DOI], [ADS], [arXiv:astro-ph/0511841]. (Cited on pages 6, 20, 32, 40,49, 50, and 53.)

Klimchuk, J. A., 2012, "The role of type II spicules in the upper solar atmosphere", J. Geophys. Res., 117(A16), A12102. [DOI], [ADS], [arXiv:1207.7048 [astro-ph.SR]]. (Cited on pages 32 and 48.)

Klimchuk, J. A. and Gary, D. E., 1995, "A Comparison of Active Region Temperatures and Emission Measures Observed in Soft X-Rays and Microwaves and Implications for Coronal Heating", Astrophys. J., 448, 925. [DOI], [ADS]. (Cited on page 20.)

Klimchuk, J. A. and Porter, L. J., 1995, "Scaling of heating rates in solar coronal loops", Nature, 377, 131-133. [DOI], [ADS]. (Cited on page 20.)

Klimchuk, J. A., Lemen, J. R., Feldman, U., Tsuneta, S. and Uchida, Y., 1992, "Thickness variations along coronal loops observed by the Soft X-ray Telescope on YOHKOH", Publ. Astron. Soc. Japan, 44, L181-L185. [ADS]. (Cited on page 14.)

Klimchuk, J. A., Patsourakos, S. and Cargill, P. J., 2008, "Highly Efficient Modeling of Dynamic Coronal Loops", Astrophys. J., 682, 1351-1362. [DOI], [ADS], [arXiv:0710.0185]. (Cited on page 37.)

Klimchuk, J. A., Karpen, J. T. and Antiochos, S. K., 2010, "Can Thermal Nonequilibrium Explain Coronal Loops?", Astrophys. J., 714, 1239-1248. [DOI], [ADS], [arXiv:0912.0953 [astro-ph.SR]]. (Cited on page 53.)

Ko, Y.-K., Doschek, G. A., Warren, H. P. and Young, P. R., 2009, "Hot Plasma in Nonflaring Active Regions Observed by the Extreme-Ultraviolet Imaging Spectrometer on Hinode", Astrophys. J., 697, 1956-1970. [DOI], [ADS], [arXiv:0903.3029]. (Cited on page 20.)

Kopp, R. A. and Poletto, G., 1993, "Coronal Heating by Nanoflares: Individual Events and Global Energetics", Astrophys. J., 418, 496. [DOI], [ADS]. (Cited on page 51.)

Kopp, R. A., Poletto, G., Noci, G. and Bruner, M., 1985, "Analysis of loop flows observed on 27 March, 1980 by the UVSP instrument during the solar maximum mission", Solar Phys., 98, 91-118. [DOI], [ADS]. (Cited on page 27.)

Kosugi, T., Matsuzaki, K., Sakao, T. et al., 2007, "The Hinode (Solar-B) Mission: An Overview", Solar Phys., 243, 3-17. [DOI], [ADS]. (Cited on page 8.)

Kramar, M., Jones, S., Davila, J., Inhester, B. and Mierla, M., 2009, "On the Tomographic Reconstruction of the 3D Electron Density for the Solar Corona from STEREO COR1 Data", Solar Phys., 259, 109-121. [DOI], [ADS]. (Cited on page 8.)

Krieger, A., Paolini, F., Vaiana, G. S. and Webb, D., 1972, "Results from OSO-IV: the Long Term Behavior of X-Ray Emitting Regions", Solar Phys., 22, 150-177. [DOI], [ADS]. (Cited on page 7.)

Krieger, A. S., 1978, "The decay of coronal loops brightened by flares and transients", Solar Phys., 56, 107-120. [DOI], [ADS]. (Cited on page 27.)

Landi, E., 2007, "Ion Temperatures in the Quiet Solar Corona", Astrophys. J., 663, 1363-1368. [DOI], [ADS]. (Cited on page 17.)

Landi, E. and Feldman, U., 2004, "Models for Solar Magnetic Loops. IV. On the Relation between Coronal and Footpoint Plasma in Active Region Loops", Astrophys. J., 611, 537-544. [DOI], [ADS]. (Cited on pages 22,26 , and 38.) 
Landi, E. and Feldman, U., 2008, "The Thermal Structure of an Active Region Observed Outside the Solar Disk", Astrophys. J., 672, 674-683. [DOI], [ADS]. (Cited on page 22.)

Landi, E. and Klimchuk, J. A., 2010, "On the Isothermality of Solar Plasmas", Astrophys. J., 723, 320-328. [DOI], [ADS]. (Cited on page 18.)

Landi, E. and Landini, M., 2004, "Models for Solar Magnetic Loops. III. Dynamic Models and Coronal Diagnostic Spectrometer Observations", Astrophys. J., 608, 1133-1147. [DOI], [ADS]. (Cited on pages 22 and 38.)

Landi, E. and Landini, M., 2005, "Models for Solar Magnetic Loops. V. A New Diagnostic Technique to Compare Loop Models and Observations", Astrophys. J., 618, 1039-1043. [DOI], [ADS]. (Cited on page 51.)

Landi, E. and Reale, F., 2013, "Prominence Plasma Diagnostics through Extreme-ultraviolet Absorption", Astrophys. J., 772, 71. [DOI], [ADS], [arXiv:1209.2934 [astro-ph.SR]]. (Cited on page 20.)

Landi, E., Landini, M., Dere, K. P., Young, P. R. and Mason, H. E., 1999, "CHIANTI - an atomic database for emission lines. III. Continuum radiation and extension of the ion database", Astron. Astrophys. Suppl., 135, 339-346. [DOI], [ADS]. (Cited on page 18.)

Landi, E., Feldman, U. and Dere, K. P., 2002, "A Comparison between Coronal Emission Lines from an Isothermal Spectrum Observed with the Coronal Diagnostic Spectrometer and CHIANTI Emissivities", Astrophys. J., 574, 495-503. [DOI], [ADS]. (Cited on page 18.)

Landi, E., Del Zanna, G., Young, P. R., Dere, K. P., Mason, H. E. and Landini, M., 2006, "CHIANTI - An Atomic Database for Emission Lines. VII. New Data for X-Rays and Other Improvements", Astrophys. J. Suppl. Ser., 162, 261-280. [DOI], [ADS]. (Cited on page 18.)

Landi, E., Miralles, M. P., Curdt, W. and Hara, H., 2009, "Physical properties of cooling plasma in quiescent active region loops", Astrophys. J., 695, 221-237. [DOI], [ADS]. (Cited on page 25.)

Landi, E., Del Zanna, G., Young, P. R., Dere, K. P. and Mason, H. E., 2012a, "CHIANTI - An Atomic Database for Emission Lines. XII. Version 7 of the Database", Astrophys. J., 744, 99. [DOI], [ADS]. (Cited on page 18.)

Landi, E., Reale, F. and Testa, P., 2012b, "Monte Carlo Markov chain DEM reconstruction of isothermal plasmas", Astron. Astrophys., 538, A111. [DOI], [ADS], [arXiv:1112.2857 [astro-ph.SR]]. (Cited on pages 18 and 19.)

Landi, E., Young, P. R., Dere, K. P., Del Zanna, G. and Mason, H. E., 2013, "CHIANTI - An Atomic Database for Emission Lines. XIII. Soft X-Ray Improvements and Other Changes", Astrophys. J., 763, 86. [DOI], [ADS]. (Cited on page 18.)

Landini, M. and Landi, E., 2002, "Models for solar magnetic loops. I. A simple theoretical model and diagnostic procedure", Astron. Astrophys., 383, 653-660. [DOI], [ADS]. (Cited on page 38.)

Landini, M. and Monsignori Fossi, B. C., 1975, "A loop model of active coronal regions", Astron. Astrophys., 42, 213-220. [ADS]. (Cited on pages 37 and 38.)

Lee, J.-Y., Barnes, G., Leka, K. D., Reeves, K. K., Korreck, K. E., Golub, L. and DeLuca, E. E., 2010, "The Role of Magnetic Topology in the Heating of Active Region Coronal Loops", Astrophys. J., 723, 1493-1506. [DOI], [ADS], [arXiv:1009.2070 [astro-ph.SR]]. (Cited on page 50.)

Lemen, J. R., Title, A. M., Akin, D. J. et al., 2012, "The Atmospheric Imaging Assembly (AIA) on the Solar Dynamics Observatory (SDO)", Solar Phys., 275, 17-40. [DOI], [ADS]. (Cited on page 8.)

Lenz, D. D., 2004, "Effects of Flow on Structure and Abundances in Multispecies Solar Coronal Loops", Astrophys. J., 604, 433-441. [DOI], [ADS]. (Cited on page 48.) 
Lenz, D. D., DeLuca, E. E., Golub, L., Rosner, R. and Bookbinder, J. A., 1999, "Temperature and Emission-Measure Profiles along Long-lived Solar Coronal Loops Observed with the Transition Region and Coronal Explorer", Astrophys. J. Lett., 517, L155-L158. [DOI], [ADS], [arXiv:astro-ph/9903491]. (Cited on pages 11, 25, 38, and 45.)

Lin, R. P., Schwartz, R. A., Kane, S. R., Pelling, R. M. and Hurley, K. C., 1984, "Solar hard X-ray microflares", Astrophys. J., 283, 421-425. [DOI], [ADS]. (Cited on page 27.)

Linsky, J. F. and Serio, S. (Eds.), 1993, Physics of Solar and Stellar Coronae: G.S. Vaiana Memorial Symposuim, Proceedings of a conference of the IAU, held in Palermo, Italy, 22 - 26 June, 1992, Astrophysics and Space Science Library, 183, Kluwer, Dordrecht; Boston. [DOI], [ADS], [Google Books]. (Cited on page 57.)

Litwin, C. and Rosner, R., 1998, "Alfvén Wave Transmission and Heating of Solar Coronal Loops", Astrophys. J., 499, 945. [DOI], [ADS]. (Cited on pages 51 and 54.)

Liu, W., Title, A. M., Zhao, J., Ofman, L., Schrijver, C. J., Aschwanden, M. J., De Pontieu, B. and Tarbell, T. D., 2011, "Direct Imaging of Quasi-periodic Fast Propagating Waves of $\sim 2000 \mathrm{~km} \mathrm{~s}^{-1}$ in the Low Solar Corona by the Solar Dynamics Observatory Atmospheric Imaging Assembly", Astrophys. J. Lett., 736, L13. [DOI], [ADS], [arXiv:1106.3150 [astro-ph.SR]]. (Cited on page 33.)

López Fuentes, M. C. and Klimchuk, J. A., 2010, "A Simple Model for the Evolution of Multi-stranded Coronal Loops", Astrophys. J., 719, 591-601. [DOI], [ADS], [arXiv:1004.2061 [astro-ph.SR]]. (Cited on pages 46,50 , and 56. .)

López Fuentes, M. C., Klimchuk, J. A. and Démoulin, P., 2006, "The Magnetic Structure of Coronal Loops Observed by TRACE", Astrophys. J., 639, 459-474. [DOI], [ADS], [arXiv:astro-ph/0507462]. (Cited on page 15.)

López Fuentes, M. C., Klimchuk, J. A. and Mandrini, C. H., 2007, "The Temporal Evolution of Coronal Loops Observed by GOES SXI", Astrophys. J., 657, 1127-1136. [DOI], [ADS], [arXiv:astro-ph/0611338]. (Cited on page 27.)

Lu, E. T. and Hamilton, R. J., 1991, "Avalanches and the distribution of solar flares", Astrophys. J. Lett., 380, L89-L92. [DOI], [ADS]. (Cited on page 46.)

Luna, M., Terradas, J., Oliver, R. and Ballester, J. L., 2010, "Transverse Oscillations of a Multi-stranded Loop", Astrophys. J., 716, 1371-1380. [DOI], [ADS], [arXiv:0912.4491 [astro-ph.SR]]. (Cited on page 46.)

Lundquist, L. L., Fisher, G. H. and McTiernan, J. M., 2008a, "Forward Modeling of Active Region Coronal Emissions. I. Methods and Testing", Astrophys. J. Suppl. Ser., 179, 509-533. [DOI], [ADS]. (Cited on page 55.)

Lundquist, L. L., Fisher, G. H., Metcalf, T. R., Leka, K. D. and McTiernan, J. M., 2008b, "Forward Modeling of Active Region Coronal Emissions. II. Implications for Coronal Heating", Astrophys. J., 689, 1388-1405. [DOI], [ADS]. (Cited on page 55.)

Mackay, D. H., Green, L. M. and van Ballegooijen, A., 2011, "Modeling the Dispersal of an Active Region: Quantifying Energy Input into the Corona", Astrophys. J., 729, 97. [DOI], [ADS], [arXiv:1102.5296 [astro-ph.SR]]. (Cited on page 50.)

MacNeice, P., 1986, "A numerical hydrodynamic model of a heated coronal loop", Solar Phys., 103, 47-66. [DOI], [ADS]. (Cited on pages 35 and 40.)

MacNeice, P., Pallavicini, R., Mason, H. E., Simnett, G. M., Antonucci, E., Shine, R. A. and Dennis, B. R., 1985, "Multiwavelength analysis of a well observed flare from SMM", Solar Phys., 99, 167-188. [DOI], [ADS]. (Cited on page 8.) 
Madjarska, M. S., Vanninathan, K. and Doyle, J. G., 2011, "Can coronal hole spicules reach coronal temperatures?", Astron. Astrophys., 532, L1. [DOI], [ADS], [arXiv:1105.1296 [astro-ph.SR]]. (Cited on page 32.)

Maggio, A., Pallavicini, R., Reale, F. and Tagliaferri, G., 2000, "Twin X-ray flares and the active corona of AB Dor observed with BeppoSAX", Astron. Astrophys., 356, 627-642. [ADS]. (Cited on page 42.)

Malanushenko, A. and Schrijver, C. J., 2013, "On the Anisotropy in Expansion of Magnetic Flux Tubes in the Solar Corona", Astrophys. J., 775, 120. [DOI], [ADS], [arXiv:1307.3440 [astro-ph.SR]]. (Cited on page 15.)

Mandrini, C. H., Démoulin, P. and Klimchuk, J. A., 2000, "Magnetic Field and Plasma Scaling Laws: Their Implications for Coronal Heating Models", Astrophys. J., 530, 999-1015. [DOI], [ADS]. (Cited on page 55.)

Mariska, J. T., Feldman, U. and Doschek, G. A., 1980, "Physical conditions in the solar atmosphere above an active region", Astrophys. J., 240, 300-305. [DOI], [ADS]. (Cited on page 18.)

Martens, P. C. H., 2010, "Scaling Laws and Temperature Profiles for Solar and Stellar Coronal Loops with Non-uniform Heating", Astrophys. J., 714, 1290-1304. [DOI], [ADS], [arXiv:0804.2241]. (Cited on page 38.)

Martens, P. C. H., van den Oord, G. H. J. and Hoyng, P., 1985, "Observations of steady anomalous magnetic heating in thin current sheets", Solar Phys., 96, 253-275. [DOI], [ADS]. (Cited on page 50.)

Martens, P. C. H., Kankelborg, C. C. and Berger, T. E., 2000, "On the Nature of the 'Moss' Observed by TRACE", Astrophys. J., 537, 471-480. [DOI], [ADS]. (Cited on page 24.)

Martens, P. C. H., Cirtain, J. W. and Schmelz, J. T., 2002, "The Inadequacy of Temperature Measurements in the Solar Corona through Narrowband Filter and Line Ratios", Astrophys. J. Lett., 577, L115-L117. [DOI], [ADS]. (Cited on page 22.)

Mason, H. E., Landi, E., Pike, C. D. and Young, P. R., 1999, "Electron density and temperature structure of two limb active regions observed by SOHO-CDS", Solar Phys., 189, 129-146. [ADS]. (Cited on page 22.)

McIntosh, S. W. and De Pontieu, B., 2012, "Estimating the "Dark" Energy Content of the Solar Corona", Astrophys. J., 761, 138. [DOI], [ADS], [arXiv:1211.4178 [astro-ph.SR]]. (Cited on page 33.)

McIntosh, S. W., De Pontieu, B., Carlsson, M., Hansteen, V., Boerner, P. and Goossens, M., 2011, "Alfvénic waves with sufficient energy to power the quiet solar corona and fast solar wind", Nature, 475, 477-480. [DOI], [ADS]. (Cited on page 33.)

McIntosh, S. W., Tian, H., Sechler, M. and De Pontieu, B., 2012, "On the Doppler Velocity of Emission Line Profiles Formed in the "Coronal Contraflow" that Is the Chromosphere-Corona Mass Cycle", Astrophys. J., 749, 60. [DOI], [ADS], [arXiv:1202.1248 [astro-ph.SR]]. (Cited on page 32.)

McLaughlin, J. A. and Ofman, L., 2008, "Three-dimensional Magnetohydrodynamic Wave Behavior in Active Regions: Individual Loop Density Structure", Astrophys. J., 682, 1338-1350. [DOI], [ADS]. (Cited on page 37.)

McLaughlin, J. A., de Moortel, I. and Hood, A. W., 2011, "Phase mixing of nonlinear visco-resistive Alfvén waves", Astron. Astrophys., 527, A149. [DOI], [ADS], [arXiv:1101.5945 [astro-ph.SR]]. (Cited on page 54.)

McTiernan, J. M., 2009, "RHESSI/GOES Observations of the Nonflaring Sun from 2002 to 2006", Astrophys. J., 697, 94-99. [DOI], [ADS]. (Cited on page 21.)

Mendoza-Briceno, C. A. and Hood, A. W., 1997, "The effect of spatially dependent heating on the thermal equilibria of coronal loops", Astron. Astrophys., 325, 791-802. [ADS]. (Cited on page 45.) 
Mendoza-Briceño, C. A., Erdélyi, R. and Di G. Sigalotti, L., 2002, "Coronal Loop Heating by Random Energy Releases", Astrophys. J. Lett., 579, L49-L52. [DOI], [ADS]. (Cited on page 51.)

Mendoza-Briceño, C. A., Sigalotti, L. D. G. and Erdélyi, R., 2005, "Catastrophic Cooling of Impulsively Heated Coronal Loops", Astrophys. J., 624, 1080-1092. [DOI], [ADS]. (Cited on page 52.)

Miceli, M., Reale, F., Gburek, S. et al., 2012, "X-ray emitting hot plasma in solar active regions observed by the SphinX spectrometer", Astron. Astrophys., 544, A139. [DOI], [ADS], [arXiv:1207.4665 [astroph.SR]]. (Cited on page 21.)

Mitra-Kraev, U. and Benz, A. O., 2001, "A nanoflare heating model for the quiet solar corona", Astron. Astrophys., 373, 318-328. [DOI], [ADS], [arXiv:astro-ph/0104218]. (Cited on page 51.)

Mok, Y., Mikić, Z., Lionello, R. and Linker, J. A., 2008, "The Formation of Coronal Loops by Thermal Instability in Three Dimensions", Astrophys. J. Lett., 679, L161-L165. [DOI], [ADS]. (Cited on page 53.)

Montesinos, B. and Thomas, J. H., 1989, "Siphon flows in isolated magnetic flux tubes. II. Adiabatic flows", Astrophys. J., 337, 977-988. [DOI], [ADS]. (Cited on page 46.)

Montesinos, B. and Thomas, J. H., 1993, "Siphon flows in isolated magnetic flux tubes. V. Radiative flows with variable ionization", Astrophys. J., 402, 314-325. [DOI], [ADS]. (Cited on page 46.)

Morales, L. and Charbonneau, P., 2008, "Self-organized Critical Model of Energy Release in an Idealized Coronal Loop", Astrophys. J., 682, 654-666. [DOI], [ADS]. (Cited on page 56.)

Morton, R. J. and McLaughlin, J. A., 2013, "Hi-C and AIA observations of transverse magnetohydrodynamic waves in active regions", Astron. Astrophys., 553, L10. [DOI], [ADS]. (Cited on page 33.)

Müller, D. A. N., Hansteen, V. H. and Peter, H., 2003, "Dynamics of solar coronal loops. I. Condensation in cool loops and its effect on transition region lines", Astron. Astrophys., 411, 605-613. [DOI], [ADS]. (Cited on page 35.)

Müller, D. A. N., Peter, H. and Hansteen, V. H., 2004, "Dynamics of solar coronal loops. II. Catastrophic cooling and high-speed downflows", Astron. Astrophys., 424, 289-300. [DOI], [ADS], [arXiv:astroph/0405538]. (Cited on pages 48, 51, and 53.)

Müller, D. A. N., De Groof, A., Hansteen, V. H. and Peter, H., 2005, "High-speed coronal rain", Astron. Astrophys., 436, 1067-1074. [DOI], [ADS]. (Cited on page 48.)

Muller, R., Roudier, T., Vigneau, J. and Auffret, H., 1994, "The proper motion of network bright points and the heating of the solar corona", Astron. Astrophys., 283, 232-240. [ADS]. (Cited on page 32.)

Nagai, F., 1980, "A model of hot loops associated with solar flares. I. Gasdynamics in the loops", Solar Phys., 68, 351-379. [DOI], [ADS]. (Cited on pages 35 and 40.)

Nagai, F. and Emslie, A. G., 1984, "Gas dynamics in the impulsive phase of solar flares. I. Thick-target heating by nonthermal electrons", Astrophys. J., 279, 896-908. [DOI], [ADS]. (Cited on pages 35 and 40.)

Nagata, S., Hara, H., Kano, R. et al., 2003, "Spatial and Temporal Properties of Hot and Cool Coronal Loops", Astrophys. J., 590, 1095-1110. [DOI], [ADS]. (Cited on page 15.)

Nakariakov, V. M. and Ofman, L., 2001, "Determination of the coronal magnetic field by coronal loop oscillations", Astron. Astrophys., 372, L53-L56. [DOI], [ADS]. (Cited on pages 12 and 33.)

Nakariakov, V. M. and Verwichte, E., 2005, "Coronal Waves and Oscillations", Living Rev. Solar Phys., 2, lrsp-2005-3. [DOI], [ADS]. URL (accessed 26 October 2010):

http://www.livingreviews.org/lrsp-2005-3. (Cited on page 53.) 
Nakariakov, V. M., Roberts, B. and Murawski, K., 1997, "Alfvén Wave Phase Mixing as a Source of Fast Magnetosonic Waves", Solar Phys., 175, 93-105. [DOI], [ADS]. (Cited on page 54.)

Nakariakov, V. M., Ofman, L., DeLuca, E. E., Roberts, B. and Davila, J. M., 1999, "TRACE observations of damped coronal loop oscillations: implications for coronal heating", Science, 285, 862-864. [DOI], [ADS]. (Cited on pages 12, 33, 50, and 54.)

Nakariakov, V. M., Verwichte, E., Berghmans, D. and Robbrecht, E., 2000, "Slow magnetoacoustic waves in coronal loops", Astron. Astrophys., 362, 1151-1157. [ADS]. (Cited on page 54.)

Narain, U. and Ulmschneider, P., 1996, "Chromospheric and Coronal Heating Mechanisms II", Space Sci. Rev., 75, 453-509. [DOI], [ADS]. (Cited on page 53.)

Nigro, G., Malara, F., Carbone, V. and Veltri, P., 2004, "Nanoflares and MHD Turbulence in Coronal Loops: A Hybrid Shell Model", Phys. Rev. Lett., 92, 194501. [DOI], [ADS]. (Cited on page 55.)

Nisticò, G., Nakariakov, V. M. and Verwichte, E., 2013, "Decaying and decayless transverse oscillations of a coronal loop", Astron. Astrophys., 552, A57. [DOI], [ADS]. (Cited on page 33.)

Nitta, N., 2000, "The relation between hot and cool loops", Solar Phys., 195, 123-133. [ADS]. (Cited on page 21.)

Nitta, S., Imada, S. and Yamamoto, T. T., 2012, "Clear Detection of Chromospheric Evaporation Upflows with High Spatial/Temporal Resolution by Hinode XRT", Solar Phys., 276, 183-197. [DOI], [ADS]. (Cited on page 32.)

Noci, G., 1981, "Siphon flows in the solar corona", Solar Phys., 69, 63-76. [DOI], [ADS]. (Cited on page 46.)

Noci, G., Spadaro, D., Zappala, R. A. and Antiochos, S. K., 1989, "Mass flows and the ionization states of coronal loops", Astrophys. J., 338, 1131-1138. [DOI], [ADS]. (Cited on page 46.)

Noglik, J. B., Walsh, R. W. and Cirtain, J. W., 2008, "Comparison of High-Resolution TRACE Data to Spectroscopic CDS Data for Temperature Determination", Astrophys. J., 674, 1191-1200. [DOI], [ADS]. (Cited on pages 25 and 26.)

O’Dwyer, B., Del Zanna, G., Mason, H. E., Sterling, A. C., Tripathi, D. and Young, P. R., 2011, "Hinode extreme-ultraviolet imaging spectrometer observations of a limb active region", Astron. Astrophys., 525, A137. [DOI], [ADS]. (Cited on page 21.)

Ofman, L., 2009, "Three-Dimensional Magnetohydrodynamic Models of Twisted Multithreaded Coronal Loop Oscillations", Astrophys. J., 694, 502-511. [DOI], [ADS]. (Cited on pages 37, 46, 50, and 54.)

Ofman, L. and Aschwanden, M. J., 2002, "Damping Time Scaling of Coronal Loop Oscillations Deduced from Transition Region and Coronal Explorer Observations", Astrophys. J. Lett., 576, L153-L156. [DOI], [ADS]. (Cited on page 54.)

Ofman, L. and Wang, T., 2002, "Hot Coronal Loop Oscillations Observed by SUMER: Slow Magnetosonic Wave Damping by Thermal Conduction", Astrophys. J. Lett., 580, L85-L88. [DOI], [ADS]. (Cited on page 35.)

Ofman, L. and Wang, T. J., 2008, "Hinode observations of transverse waves with flows in coronal loops", Astron. Astrophys., 482, L9-L12. [DOI], [ADS]. (Cited on pages 32, 45, and 54.)

Ofman, L., Davila, J. M. and Steinolfson, R. S., 1995, "Coronal heating by the resonant absorption of Alfvén waves: Wavenumber scaling laws", Astrophys. J., 444, 471-477. [DOI], [ADS]. (Cited on page 54.) 
Ofman, L., Klimchuk, J. A. and Davila, J. M., 1998, "A Self-consistent Model for the Resonant Heating of Coronal Loops: The Effects of Coupling with the Chromosphere", Astrophys. J., 493, 474-479. [DOI], [ADS]. (Cited on page 54.)

Ofman, L., Liu, W., Title, A. and Aschwanden, M., 2011, "Modeling Super-fast Magnetosonic Waves Observed by SDO in Active Region Funnels", Astrophys. J. Lett., 740, L33. [DOI], [ADS]. (Cited on page 33.)

Ofman, L., Wang, T. J. and Davila, J. M., 2012, "Slow Magnetosonic Waves and Fast Flows in Active Region Loops", Astrophys. J., 754, 111. [DOI], [ADS], [arXiv:1205.5732 [astro-ph.SR]]. (Cited on pages 32 and 55.)

Ogawara, Y., Takano, T., Kato, T., Kosugi, T., Tsuneta, S., Watanabe, T., Kondo, I. and Uchida, Y., 1991, "The SOLAR-A Mission: An Overview", Solar Phys., 136, 1-16. [DOI], [ADS]. (Cited on page 8.)

O'Neill, I. and Li, X., 2005, "Coronal loops heated by turbulence-driven Alfvén waves: A two fluid model", Astron. Astrophys., 435, 1159-1167. [DOI], [ADS]. (Cited on pages 48 and 54.)

Oraevsky, V. N. and Sobelman, I. I., 2002, "Comprehensive studies of solar activity on the CORONAS-F satellite", Astron. Lett., 28, 401-410. [DOI], [ADS]. (Cited on page 8.)

Orlando, S. and Peres, G., 1999, "Effects on UV lines observations of stationary plasma flows confined in coronal loops", Phys. Chem. Earth, 24, 401-406. [DOI], [ADS]. (Cited on pages 46 and 47.)

Orlando, S., Peres, G. and Serio, S., 1995a, "Models of stationary siphon flows in stratified, thermally conducting coronal loops II. Shocked solutions", Astron. Astrophys., 300, 549. [ADS]. (Cited on page 46.)

Orlando, S., Peres, G. and Serio, S., 1995b, "Models of stationary siphon flows in stratified, thermally conducting coronal loops I. Regular solutions", Astron. Astrophys., 294, 861-873. [ADS]. (Cited on page 46.)

Orlando, S., Peres, G. and Reale, F., 2000, "The Sun as an X-Ray Star. I. Deriving the Emission Measure Distribution versus Temperature of the Whole Solar Corona from the Yohkoh/Soft X-Ray Telescope Data", Astrophys. J., 528, 524-536. [DOI], [ADS]. (Cited on pages 51 and 57.)

Orlando, S., Peres, G. and Reale, F., 2004, "The Sun as an X-ray star: Active region evolution, rotational modulation, and implications for stellar X-ray variability", Astron. Astrophys., 424, 677-689. [DOI], [ADS]. (Cited on page 57.)

O'Shea, E., Banerjee, D. and Doyle, J. G., 2007, "Plasma condensation in coronal loops", Astron. Astrophys., 475, L25-L28. [DOI], [ADS]. (Cited on page 27.)

Parenti, S. and Young, P. R., 2008, "On the ultraviolet signatures of small scale heating in coronal loops", Astron. Astrophys., 492, 857-862. [DOI], [ADS]. (Cited on page 52.)

Parenti, S., Buchlin, E., Cargill, P. J., Galtier, S. and Vial, J.-C., 2006, "Modeling the Radiative Signatures of Turbulent Heating in Coronal Loops", Astrophys. J., 651, 1219-1228. [DOI], [ADS]. (Cited on page 50.)

Parker, E. N., 1988, "Nanoflares and the solar X-ray corona", Astrophys. J., 330, 474-479. [DOI], [ADS]. (Cited on pages 45, 49, 51, and 55.)

Parker, E. N., 1991, "Heating solar coronal holes", Astrophys. J., 372, 719-727. [DOI], [ADS]. (Cited on page 53.)

Parnell, C. E. and De Moortel, I., 2012, "A contemporary view of coronal heating", Philos. Trans. R. Soc. London, Ser. A, 370, 3217-3240. [DOI], [ADS], [arXiv:1206.6097 [astro-ph.SR]]. (Cited on page 6.) 
Pascoe, D. J., de Moortel, I. and McLaughlin, J. A., 2009, "Impulsively generated oscillations in a 3D coronal loop", Astron. Astrophys., 505, 319-327. [DOI], [ADS]. (Cited on page 37.)

Patsourakos, S. and Klimchuk, J. A., 2005, "Coronal Loop Heating by Nanoflares: The Impact of the Field-aligned Distribution of the Heating on Loop Observations", Astrophys. J., 628, 1023-1030. [DOI], [ADS]. (Cited on page 51.)

Patsourakos, S. and Klimchuk, J. A., 2006, "Nonthermal Spectral Line Broadening and the Nanoflare Model", Astrophys. J., 647, 1452-1465. [DOI], [ADS]. (Cited on pages 46 and 54.)

Patsourakos, S. and Klimchuk, J. A., 2007, "The Cross-Field Thermal Structure of Coronal Loops from Triple-Filter TRACE Observations", Astrophys. J., 667, 591-601. [DOI], [ADS]. (Cited on pages 17, 25, and 26.)

Patsourakos, S. and Klimchuk, J. A., 2008, "Static and Impulsive Models of Solar Active Regions", Astrophys. J., 689, 1406-1411. [DOI], [ADS], [arXiv:0808.2745]. (Cited on page 51.)

Patsourakos, S. and Klimchuk, J. A., 2009, "Spectroscopic Observations of Hot Lines Constraining Coronal Heating in Solar Active Regions", Astrophys. J., 696, 760-765. [DOI], [ADS], [arXiv:0903.3880]. (Cited on page 20.)

Patsourakos, S., Klimchuk, J. A. and MacNeice, P. J., 2004, "The Inability of Steady-Flow Models to Explain the Extreme-Ultraviolet Coronal Loops", Astrophys. J., 603, 322-329. [DOI], [ADS]. (Cited on pages 45 and 48 .)

Pekünlü, E. R., Çakırlı, Ö. and Özetken, E., 2001, "Solar coronal heating by magnetosonic waves", Mon. Not. R. Astron. Soc., 326, 675-685. [DOI], [ADS]. (Cited on page 54.)

Peres, G. and Vaiana, G. S., 1990, "X-ray observations, scaling laws and magnetic fields", Mem. Soc. Astron. Ital., 61, 401-430. [ADS]. (Cited on pages 6 and 7.)

Peres, G., Serio, S., Vaiana, G. S. and Rosner, R., 1982, "Coronal closed structures. IV. Hydrodynamical stability and response to heating perturbations", Astrophys. J., 252, 791-799. [DOI], [ADS]. (Cited on pages 35,40 , and 55.)

Peres, G., Spadaro, D. and Noci, G., 1992, "Steady siphon flows in closed coronal structures: Comparison with extreme-ultraviolet observations", Astrophys. J., 389, 777-783. [DOI], [ADS]. (Cited on page 46.)

Peres, G., Reale, F. and Serio, S., 1993, "Hydrodynamics and Diagnostics of Coronal Loops Subject to Dynamic Heating", in Physics of Solar and Stellar Coronae: G.S. Vaiana Memorial Symposuim, Proceedings of a conference of the IAU, held in Palermo, Italy, 22-26 June, (Eds.) Linsky, J. L., Serio, S., Astrophysics and Space Science Library, 183, p. 151, Kluwer, Dordrecht; Boston. [ADS]. (Cited on page 51.)

Peres, G., Reale, F. and Golub, L., 1994, "Loop models of low coronal structures observed by the Normal Incidence X-Ray Telescope (NIXT)", Astrophys. J., 422, 412-415. [DOI], [ADS]. (Cited on pages 21 and 24.)

Peres, G., Orlando, S., Reale, F., Rosner, R. and Hudson, H. S., 2000, "The Sun as an X-Ray Star. II. Using the Yohkoh/Soft X-Ray Telescope-derived Solar Emission Measure versus Temperature to Interpret Stellar X-Ray Observations", Astrophys. J., 528, 537-551. [DOI], [ADS]. (Cited on pages 9, 25, and 57.)

Peres, G., Orlando, S., Reale, F. and Rosner, R., 2001, "The Distribution of the Emission Measure, and of the Heating Budget, among the Loops in the Corona", Astrophys. J., 563, 1045-1054. [DOI], [ADS], [arXiv:astro-ph/0111192]. (Cited on pages 51 and 57.)

Peres, G., Orlando, S. and Reale, F., 2004, "Are Coronae of Late-Type Stars Made of Solar-like Structures? The X-Ray Surface Flux versus Hardness Ratio Diagram and the Pressure-Temperature Correlation", Astrophys. J., 612, 472-480. [DOI], [ADS], [arXiv:astro-ph/0405281]. (Cited on page 57.) 
Pesnell, W. D., Thompson, B. J. and Chamberlin, P. C., 2012, "The Solar Dynamics Observatory (SDO)", Solar Phys., 275, 3-15. [DOI], [ADS]. (Cited on page 8.)

Peter, H., 1999, "Analysis of Transition-Region Emission-Line Profiles from Full-Disk Scans of the Sun Using the SUMER Instrument on SOHO", Astrophys. J., 516, 490-504. [DOI], [ADS]. (Cited on page 29.)

Peter, H. and Bingert, S., 2012, "Constant cross section of loops in the solar corona", Astron. Astrophys., 548, A1. [DOI], [ADS], [arXiv:1209.0789 [astro-ph.SR]]. (Cited on pages 15 and 37.)

Peter, H., Bingert, S. and Kamio, S., 2012, "Catastrophic cooling and cessation of heating in the solar corona", Astron. Astrophys., 537, A152. [DOI], [ADS], [arXiv:1112.3667 [astro-ph.SR]]. (Cited on page 53.)

Peter, H., Bingert, S., Klimchuk, J. A. et al., 2013, "Structure of solar coronal loops: from miniature to large-scale", Astron. Astrophys., 556, A104. [DOI], [ADS], [arXiv:1306.4685 [astro-ph.SR]]. (Cited on page 16.)

Peterson, W. M., Mutel, R. L., Güdel, M. and Goss, W. M., 2010, "A large coronal loop in the Algol system", Nature, 463, 207-209. [DOI], [ADS]. (Cited on page 57.)

Poletto, G., Vaiana, G. S., Zombeck, M. V., Krieger, A. S. and Timothy, A. F., 1975, "A comparison of coronal X-ray structures of active regions with magnetic fields computed from photospheric observations", Solar Phys., 44, 83-99. [DOI], [ADS]. (Cited on page 7.)

Pontin, D. I., Wilmot-Smith, A. L., Hornig, G. and Galsgaard, K., 2011, "Dynamics of braided coronal loops. II. Cascade to multiple small-scale reconnection events", Astron. Astrophys., 525, A57. [DOI], [ADS], [arXiv:1003.5784 [astro-ph.SR]]. (Cited on page 50.)

Porter, L. J. and Klimchuk, J. A., 1995, "Soft X-Ray Loops and Coronal Heating", Astrophys. J., 454, 499. [DOI], [ADS]. (Cited on pages 11 and 20.)

Porter, L. J., Klimchuk, J. A. and Sturrock, P. A., 1994, "The possible role of MHD waves in heating the solar corona", Astrophys. J., 435, 482-501. [DOI], [ADS]. (Cited on page 54.)

Pottasch, S. R., 1963, "The Lower Solar Corona: Interpretation of the Ultraviolet Spectrum", Astrophys. J., 137, 945-966. [DOI], [ADS]. (Cited on page 18.)

Priest, E. R., 1978, "The structure of coronal loops", Solar Phys., 58, 57-87. [DOI], [ADS]. (Cited on page 34.)

Priest, E. R., 1981, "Theory of loop flows and instability", in Solar Active Regions, A Monograph from Skylab Solar Workshop III, (Ed.) Orrall, F. Q., pp. 213-275, Colorado Associated University Press, Boulder, CO. [ADS]. (Cited on page 46.)

Priest, E. R., 2011, "The Flux Tube Tectonics model for coronal heating", J. Atmos. Sol.-Terr. Phys., 73, 271-276. [DOI], [ADS]. (Cited on page 51.)

Priest, E. R., Foley, C. R., Heyvaerts, J., Arber, T. D., Mackay, D. H., Culhane, J. L. and Acton, L. W., 2000, "A Method to Determine the Heating Mechanisms of the Solar Corona", Astrophys. J., 539 1002-1022. [DOI], [ADS]. (Cited on pages 18 and 50.)

Priest, E. R., Heyvaerts, J. F. and Title, A. M., 2002, "A Flux-Tube Tectonics Model for Solar Coronal Heating Driven by the Magnetic Carpet", Astrophys. J., 576, 533-551. [DOI], [ADS]. (Cited on pages 45, 49, 50, and 51.)

Rappazzo, A. F. and Velli, M., 2011, "Magnetohydrodynamic turbulent cascade of coronal loop magnetic fields", Phys. Rev. E, 83(6), 065401. [DOI], [ADS], [arXiv:1005.1640 [astro-ph.SR]]. (Cited on page 56.) 
Rappazzo, A. F., Velli, M., Einaudi, G. and Dahlburg, R. B., 2007, "Coronal Heating, Weak MHD Turbulence, and Scaling Laws", Astrophys. J. Lett., 657, L47-L51. [DOI], [ADS], [arXiv:astro-ph/0701872]. (Cited on page 56.)

Rappazzo, A. F., Velli, M., Einaudi, G. and Dahlburg, R. B., 2008, "Nonlinear Dynamics of the Parker Scenario for Coronal Heating", Astrophys. J., 677, 1348-1366. [DOI], [ADS], [arXiv:0709.3687]. (Cited on page 56.)

Rappazzo, A. F., Velli, M. and Einaudi, G., 2010, "Shear Photospheric Forcing and the Origin of Turbulence in Coronal Loops", Astrophys. J., 722, 65-78. [DOI], [ADS], [arXiv:1003.3872 [astro-ph.SR]]. (Cited on page 56.)

Raymond, J. C., Cox, D. P. and Smith, B. W., 1976, "Radiative cooling of a low-density plasma", Astrophys. J., 204, 290-292. [DOI], [ADS]. (Cited on page 35.)

Reale, F., 1999, "Inclination of large coronal loops observed by TRACE", Solar Phys., 190, 139-144. [DOI], [ADS]. (Cited on page 38.)

Reale, F., 2002a, "Stellar Flare Modeling", in Stellar Coronae in the Chandra and XMM-NEWTON Era, Proceedings of a symposium held at ESTEC, Noordwijk, The Netherlands, 25-29 June 2001, (Eds.) Favata, F., Drake, J. J., ASP Conference Series, 277, p. 103, Astronomical Society of the Pacific, San Francisco. [ADS]. (Cited on pages 18 and 57.)

Reale, F., 2002b, "More on the Determination of the Coronal Heating Function from Yohkoh Data", Astrophys. J., 580, 566-573. [DOI], [ADS], [arXiv:astro-ph/0207550]. (Cited on page 50.)

Reale, F., 2003, "Modeling solar and stellar flares", Adv. Space Res., 32, 1057-1066. [DOI], [ADS]. (Cited on page 57.)

Reale, F., 2005, "Sub-Structuring Dynamics and Heating in Dense Coronal Structures", in The Dynamic Sun: Challenges for Theory and Observations, Proceedings of the 11th European Solar Physics Meeting, 11 - 16 September 2005, Leuven, Belgium, (Eds.) Danesy, D., Poedts, S., De Groof, A., Andries, J., ESA Special Publications, SP-600, ESA Publications Division, Noordwijk. [ADS]. (Cited on page 6.)

Reale, F., 2007, "Diagnostics of stellar flares from X-ray observations: from the decay to the rise phase", Astron. Astrophys., 471, 271-279. [DOI], [ADS], [arXiv:0705.3254]. (Cited on pages 40, 42, 43, and 44.)

Reale, F. and Ciaravella, A., 2006, "Analysis of a multi-wavelength time-resolved observation of a coronal loop", Astron. Astrophys., 449, 1177-1192. [DOI], [ADS], [arXiv:astro-ph/0512397]. (Cited on pages 18, 22, and 26.)

Reale, F. and Orlando, S., 2008, "Nonequilibrium of Ionization and the Detection of Hot Plasma in Nanoflare-heated Coronal Loops", Astrophys. J., 684, 715-724. [DOI], [ADS], [arXiv:0805.3512]. (Cited on pages 36 and 40.)

Reale, F. and Peres, G., 2000, "TRACE-derived Temperature and Emission Measure Profiles along Longlived Coronal Loops: The Role of Filamentation", Astrophys. J. Lett., 528, L45-L48. [DOI], [ADS], [arXiv:astro-ph/9911096]. (Cited on pages 36 and 45.)

Reale, F., Peres, G., Serio, S., Rosner, R. and Schmitt, J. H. M. M., 1988, "Hydrodynamic modeling of an X-ray flare on Proxima Centauri observed by the Einstein telescope", Astrophys. J., 328, 256-264. [DOI], [ADS]. (Cited on page 57.)

Reale, F., Serio, S. and Peres, G., 1993, "Dynamics of the decay of confined stellar X-ray flares", Astron. Astrophys., 272, 486. [ADS]. (Cited on page 41.)

Reale, F., Peres, G. and Serio, S., 1996, "Radiatively-driven downdrafts and redshifts in transition region lines. I. Reference model", Astron. Astrophys., 316, 215-228. [ADS]. (Cited on pages 29 and 46.) 
Reale, F., Betta, R., Peres, G., Serio, S. and McTiernan, J., 1997a, "Determination of the length of coronal loops from the decay of X-ray flares I. Solar flares observed with YOHKOH SXT", Astron. Astrophys., 325, 782-790. [ADS]. (Cited on page 41.)

Reale, F., Peres, G. and Serio, S., 1997b, "Radiatively driven downdrafts and redshifts in transition region lines. II. Exploring the parameter space", Astron. Astrophys., 318, 506-520. [ADS]. (Cited on pages 29 and 46.)

Reale, F., Peres, G., Serio, S., Betta, R. M., DeLuca, E. E. and Golub, L., 2000a, "A Brightening Coronal Loop Observed by TRACE. II. Loop Modeling and Constraints on Heating", Astrophys. J., 535, 423437. [DOI], [ADS]. (Cited on pages 13, 27, 35, and 50.)

Reale, F., Peres, G., Serio, S., DeLuca, E. E. and Golub, L., 2000b, "A Brightening Coronal Loop Observed by TRACE. I. Morphology and Evolution", Astrophys. J., 535, 412-422. [DOI], [ADS]. (Cited on pages 13,27 , and 50.)

Reale, F., Peres, G. and Orlando, S., 2001, "The Sun as an X-Ray Star. III. Flares", Astrophys. J., 557, 906-920. [DOI], [ADS], [arXiv:astro-ph/0104021]. (Cited on page 57.)

Reale, F., Bocchino, F. and Peres, G., 2002, "Modeling non-confined coronal flares: Dynamics and X-ray diagnostics", Astron. Astrophys., 383, 952-971. [DOI], [ADS], [arXiv:astro-ph/0112333]. (Cited on page 57.)

Reale, F., Güdel, M., Peres, G. and Audard, M., 2004, "Modeling an X-ray flare on Proxima Centauri: Evidence of two flaring loop components and of two heating mechanisms at work", Astron. Astrophys., 416, 733-747. [DOI], [ADS], [arXiv:astro-ph/0312267]. (Cited on page 57.)

Reale, F., Nigro, G., Malara, F., Peres, G. and Veltri, P., 2005, "Modeling a Coronal Loop Heated by Magnetohydrodynamic Turbulence Nanoflares", Astrophys. J., 633, 489-498. [DOI], [ADS], [arXiv:astroph/0506694]. (Cited on pages 50, 51, 55, and 56.)

Reale, F., Parenti, S., Reeves, K. K. et al., 2007, "Fine Thermal Structure of a Coronal Active Region", Science, 318, 1582. [DOI], [ADS]. (Cited on page 21.)

Reale, F., McTiernan, J. M. and Testa, P., 2009a, "Comparison of Hinode/XRT and RHESSI Detection of Hot Plasma in the Non-Flaring Solar Corona", Astrophys. J. Lett., 704, L58-L61. [DOI], [ADS], [arXiv:0909.2529]. (Cited on pages 9, 17, 21, and 25.)

Reale, F., Testa, P., Klimchuk, J. A. and Parenti, S., 2009b, "Evidence of Widespread Hot Plasma in a Nonflaring Coronal Active Region from Hinode/X-Ray Telescope", Astrophys. J., 698, 756-765. [DOI], [ADS], [arXiv:0904.0878]. (Cited on page 21.)

Reale, F., Guarrasi, M., Testa, P., DeLuca, E. E., Peres, G. and Golub, L., 2011, "Solar Dynamics Observatory Discovers Thin High Temperature Strands in Coronal Active Regions", Astrophys. J. Lett., 736, L16. [DOI], [ADS], [arXiv:1106.1591 [astro-ph.SR]]. (Cited on pages 16, 21, and 45.)

Reale, F., Landi, E. and Orlando, S., 2012, "Post-flare Ultraviolet Light Curves Explained with Thermal Instability of Loop Plasma", Astrophys. J., 746, 18. [DOI], [ADS], [arXiv:1111.3579 [astro-ph.SR]]. (Cited on pages 40 and 53.)

Reale, F., Orlando, S., Testa, P., Peres, G., Landi, E. and Schrijver, C. J., 2013, "Bright Hot Impacts by Erupted Fragments Falling Back on the Sun: A Template for Stellar Accretion", Science, 341, 251-253. [DOI], [ADS]. (Cited on page 57.)

Reep, J. W., Bradshaw, S. J. and Klimchuk, J. A., 2013, "Diagnosing the Time Dependence of Active Region Core Heating from the Emission Measure. II. Nanoflare Trains", Astrophys. J., 764, 193. [DOI], [ADS], [arXiv:1303.4466 [astro-ph.SR]]. (Cited on page 53.) 
Reeves, E. M., Timothy, J. G. and Huber, M. C. E., 1977, "Extreme UV spectroheliometer on the Apollo Telescope Mount", Appl. Opt., 16, 837-848. [ADS]. (Cited on page 7.)

Reeves, K. K. and Warren, H. P., 2002, "Modeling the Cooling of Postflare Loops", Astrophys. J., 578, 590-597. [DOI], [ADS]. (Cited on page 40.)

Reidy, W. P., Vaiana, G. S., Zehnpfennig, T. and Giacconi, R., 1968, "Study of X-Ray Images of the Sun at Solar Minimum", Astrophys. J., 151, 333. [DOI], [ADS]. (Cited on page 7.)

Rosner, R., Tucker, W. H. and Vaiana, G. S., 1978, "Dynamics of the quiescent solar corona", Astrophys. J., 220, 643-665. [DOI], [ADS]. (Cited on pages 7, 26, 34, and 37.)

Rosner, R., Golub, L. and Vaiana, G. S., 1985, "On stellar X-ray emission", Annu. Rev. Astron. Astrophys., 23, 413-452. [DOI], [ADS]. (Cited on page 57.)

Rottman, G. J., Hassler, D. M., Jones, M. D. and Orrall, F. Q., 1990, "The systematic radial downflow in the transition region of the quiet sun from limb-to-limb observations of the C IV resonance lines", Astrophys. J., 358, 693-697. [DOI], [ADS]. (Cited on page 29.)

Saito, K. and Billings, D. E., 1964, "Polarimetric Observations of a Coronal Condensation", Astrophys. J., 140, 760. [DOI], [ADS]. (Cited on page 13.)

Sakamoto, Y., Tsuneta, S. and Vekstein, G., 2008, "Observational Appearance of Nanoflares with SXT and TRACE", Astrophys. J., 689, 1421-1432. [DOI], [ADS]. (Cited on pages 28 and 52.)

Sakamoto, Y., Tsuneta, S. and Vekstein, G., 2009, "A Nanoflare Heating Model and Comparison with Observations", Astrophys. J., 703, 2118-2130. [DOI], [ADS]. (Cited on page 52.)

Sakurai, T., 1981, "Calculation of force-free magnetic field with non-constant $\alpha$ ", Solar Phys., 69, 343-359. [DOI], [ADS]. (Cited on page 7.)

Sandman, A. W., Aschwanden, M. J., DeRosa, M. L., Wülser, J.-P. and Alexander, D., 2009, "Comparison of STEREO/EUVI loops with potential magnetic field models", Solar Phys., 259, 1-11. [DOI], [ADS]. (Cited on page 14.)

Sanz-Forcada, J., Brickhouse, N. S. and Dupree, A. K., 2003, "The Structure of Stellar Coronae in Active Binary Systems", Astrophys. J. Suppl. Ser., 145, 147-179. [DOI], [ADS], [arXiv:astro-ph/0210652]. (Cited on page 57.)

Sarro, L. M., Erdélyi, R., Doyle, J. G. and Pérez, M. E., 1999, "Modelling explosive events in the solar atmosphere", Astron. Astrophys., 351, 721-732. [ADS]. (Cited on page 46.)

Sasso, C., Andretta, V., Spadaro, D. and Susino, R., 2012, "Solar low-lying cool loops and their contribution to the transition region EUV output", Astron. Astrophys., 537, A150. [DOI], [ADS], [arXiv:1112.0309 [astro-ph.SR]]. (Cited on page 53.)

Scelsi, L., Maggio, A., Peres, G. and Pallavicini, R., 2005, "Coronal properties of G-type stars in different evolutionary phases", Astron. Astrophys., 432, 671-685. [DOI], [ADS], [arXiv:astro-ph/0501631]. (Cited on page 57.)

Scherrer, P. H., Schou, J., Bush, R. I. et al., 2012, "The Helioseismic and Magnetic Imager (HMI) Investigation for the Solar Dynamics Observatory (SDO)", Solar Phys., 275, 207-227. [DOI], [ADS]. (Cited on page 8.)

Schmelz, J. T., 2002, "Are Coronal Loops Isothermal?", Astrophys. J. Lett., 578, L161-L164. [DOI], [ADS]. (Cited on page 22.)

Schmelz, J. T., Scopes, R. T., Cirtain, J. W., Winter, H. D. and Allen, J. D., 2001, "Observational Constraints on Coronal Heating Models Using Coronal Diagnostics Spectrometer and Soft X-Ray Telescope Data", Astrophys. J., 556, 896-904. [DOI], [ADS]. (Cited on pages 22 and 25.) 
Schmelz, J. T., Beene, J. E., Nasraoui, K., Blevins, H. T., Martens, P. C. H. and Cirtain, J. W., 2003, "The Effect of Background Subtraction on the Temperature of EIT Coronal Loops", Astrophys. J., 599, 604-614. [DOI], [ADS]. (Cited on pages 18 and 22.)

Schmelz, J. T., Nasraoui, K., Richardson, V. L., Hubbard, P. J., Nevels, C. R. and Beene, J. E., 2005, "All Coronal Loops Are the Same: Evidence to the Contrary", Astrophys. J. Lett., 627, L81-L84. [DOI], [ADS], [arXiv:astro-ph/0505593]. (Cited on pages 22 and 23.)

Schmelz, J. T., Nasraoui, K., Del Zanna, G., Cirtain, J. W., DeLuca, E. E. and Mason, H. E., 2007, "Coronal Diagnostic Spectrometer Observations of Isothermal and Multithermal Coronal Loops", Astrophys. J. Lett., 658, L119-L122. [DOI], [ADS]. (Cited on pages 22 and 25.)

Schmelz, J. T., Scott, J. and Rightmire, L. A., 2008, "May Day! Coronal Loop Temperatures from the Hinode EUV Imaging Spectrometer", Astrophys. J. Lett., 684, L115-L118. [DOI], [ADS]. (Cited on page 22.)

Schmelz, J. T., Saar, S. H., DeLuca, E. E., Golub, L., Kashyap, V. L., Weber, M. A. and Klimchuk, J. A., 2009, "Hinode X-Ray Telescope Detection of Hot Emission from Quiescent Active Regions: A Nanoflare Signature?", Astrophys. J. Lett., 693, L131-L135. [DOI], [ADS], [arXiv:0901.3122]. (Cited on pages 21 and 25.)

Schmidt, H. U., 1964, "On the Observable Effects of Magnetic Energy Storage and Release Connected With Solar Flares", in The Physics of Solar Flares, Proceedings of the AAS-NASA Symposium held 28-30 October, 1963 at the Goddard Space Flight Center, Greenbelt, MD, (Ed.) Hess, W. N., NASA Special Publication, SP-50, p. 107, NASA, Washington, DC. [ADS]. (Cited on page 7.)

Schmieder, B., Rust, D. M., Georgoulis, M. K., Démoulin, P. and Bernasconi, P. N., 2004, "Emerging Flux and the Heating of Coronal Loops", Astrophys. J., 601, 530-545. [DOI], [ADS]. (Cited on page 15.)

Schmitt, J. H. M. M., Collura, A., Sciortino, S., Vaiana, G. S., Harnden Jr, F. R. and Rosner, R., 1990, "Einstein Observatory coronal temperatures of late-type stars", Astrophys. J., 365, 704-728. [DOI], [ADS]. (Cited on page 57.)

Schrijver, C. J., 2007, "Braiding-induced Interchange Reconnection of the Magnetic Field and the Width of Solar Coronal Loops", Astrophys. J. Lett., 662, L119-L122. [DOI], [ADS]. (Cited on page 15.)

Schrijver, C. J., Title, A. M., Harvey, K. L. et al., 1998, "Large-scale coronal heating by the small-scale magnetic field of the Sun", Nature, 394, 152-154. [DOI], [ADS]. (Cited on page 49.)

Schrijver, C. J., Sandman, A. W., Aschwanden, M. J. and DeRosa, M. L., 2004, "The Coronal Heating Mechanism as Identified by Full-Sun Visualizations", Astrophys. J., 615, 512-525. [DOI], [ADS]. (Cited on page 55.)

Seaton, D. B., Winebarger, A. R., DeLuca, E. E., Golub, L., Reeves, K. K. and Gallagher, P. T., 2001, "Active Region Transient Events Observed with TRACE", Astrophys. J. Lett., 563, L173-L177. [DOI], [ADS]. (Cited on page 27.)

Selwa, M. and Ofman, L., 2009, "3-D numerical simulations of coronal loops oscillations", Ann. Geophys., 27, 3899-3908. [DOI], [ADS]. (Cited on page 37.)

Serio, S., Peres, G., Vaiana, G. S., Golub, L. and Rosner, R., 1981, "Closed coronal structures. II. Generalized hydrostatic model", Astrophys. J., 243, 288-300. [DOI], [ADS]. (Cited on pages 36, 38, and 39.)

Serio, S., Reale, F., Jakimiec, J., Sylwester, B. and Sylwester, J., 1991, "Dynamics of flaring loops I. Thermodynamic decay scaling laws", Astron. Astrophys., 241, 197-202. [ADS]. (Cited on page 42.)

Sheeley Jr, N. R., 1980, "Temporal variations of loop structures in the solar atmosphere", Solar Phys., 66 , 79-87. [DOI], [ADS]. (Cited on page 27.) 
Shestov, S. V., Kuzin, S. V., Urnov, A. M., Ul'Yanov, A. S. and Bogachev, S. A., 2010, "Solar plasma temperature diagnostics in flares and active regions from spectral lines in the range $280-330 \AA$ in the SPIRIT/CORONAS-F experiment", Astron. Lett., 36, 44-58. [DOI], [ADS]. (Cited on page 21.)

Shimizu, T., 1995, "Energetics and Occurrence Rate of Active-Region Transient Brightenings and Implications for the Heating of the Active-Region Corona", Publ. Astron. Soc. Japan, 47, 251-263. [ADS]. (Cited on page 51.)

Shimizu, T. and Tsuneta, S., 1997, "Deep Survey of Solar Nanoflares with YOHKOH", Astrophys. J., 486, 1045. [DOI], [ADS]. (Cited on page 52.)

Shimizu, T., Tsuneta, S., Acton, L. W., Lemen, J. R., Ogawara, Y. and Uchida, Y., 1994, "Morphology of active region transient brightenings with the YOHKOH Soft X-ray Telescope", Astrophys. J., 422, 906-911. [DOI], [ADS]. (Cited on page 27.)

Shimojo, M., Kurokawa, H. and Yoshimura, K., 2002, "Dynamical Features and Evolutional Characteristics of Brightening Coronal Loops", Solar Phys., 206, 133-142. [DOI], [ADS]. (Cited on page 27.)

Sigalotti, L. D. G. and Mendoza-Briceño, C. A., 2003, "Dynamics of solar coronal loops", Astron. Astrophys., 397, 1083-1095. [DOI], [ADS]. (Cited on page 35.)

Sokolov, I. V., van der Holst, B., Oran, R. et al., 2013, "Magnetohydrodynamic Waves and Coronal Heating: Unifying Empirical and MHD Turbulence Models", Astrophys. J., 764, 23. [DOI], [ADS], [arXiv:1208.3141 [astro-ph.SR]]. (Cited on page 37.)

Soler, R., Andries, J. and Goossens, M., 2012, "Resonant Alfvén waves in partially ionized plasmas of the solar atmosphere", Astron. Astrophys., 537, A84. [DOI], [ADS], [arXiv:1111.4134 [astro-ph.SR]]. (Cited on page 54.)

Spadaro, D., Noci, G., Zappala, R. A. and Antiochos, S. K., 1990, "The effect of nonequilibrium ionization on ultraviolet line shifts in the solar transition region", Astrophys. J., 355, 342-347. [DOI], [ADS]. (Cited on page 46.)

Spadaro, D., Lanza, A. F., Lanzafame, A. C., Karpen, J. T., Antiochos, S. K., Klimchuk, J. A. and MacNeice, P. J., 2003, "A Transient Heating Model for Coronal Structure and Dynamics", Astrophys. J., 582, 486-494. [DOI], [ADS]. (Cited on page 51.)

Spitzer, L., 1962, Physics of Fully Ionized Gases, Interscience Tracts on Physics and Astronomy, 3, Interscience, New York, 2nd rev. edn. [ADS]. (Cited on page 35.)

Steinolfson, R. S. and Davila, J. M., 1993, "Coronal heating by the resonant absorption of Alfvén waves: Importance of the global mode and scaling laws", Astrophys. J., 415, 354-363. [DOI], [ADS]. (Cited on page 53.)

Stelzer, B., Burwitz, V., Audard, M. et al., 2002, "Simultaneous X-ray spectroscopy of YY Gem with Chandra and XMM-Newton", Astron. Astrophys., 392, 585-598. [DOI], [ADS], [arXiv:astro-ph/0206429]. (Cited on page 42.)

Strong, K. T., Harvey, K., Hirayama, T., Nitta, N., Shimizu, T. and Tsuneta, S., 1992, "Observations of the variability of coronal bright points by the Soft X-ray Telescope on YOHKOH", Publ. Astron. Soc. Japan, 44, L161-L166. [ADS]. (Cited on page 27.)

Susino, R., Lanzafame, A. C., Lanza, A. F. and Spadaro, D., 2010, "Signatures of Impulsive Localized Heating in the Temperature Distribution of Multi-Stranded Coronal Loops", Astrophys. J., 709, 499506. [DOI], [ADS], [arXiv:0912.2592 [astro-ph.SR]]. (Cited on page 52.)

Sylwester, B., Sylwester, J., Serio, S., Reale, F., Bentley, R. D. and Fludra, A., 1993, "Dynamics of flaring loops III. Interpretation of flare evolution in the emission measure-temperature diagram", Astron. Astrophys., 267, 586-594. [ADS]. (Cited on pages 41 and 42.) 
Sylwester, B., Sylwester, J. and Phillips, K. J. H., 2010, "Soft X-ray coronal spectra at low activity levels observed by RESIK", Astron. Astrophys., 514, A82. [DOI], [ADS], [arXiv:1003.2980 [astro-ph.SR]]. (Cited on page 21.)

Sylwester, J., Gaicki, I., Kordylewski, Z. et al., 1998, "RESIK: High Sensitivity Soft X-ray Spectrometer for the Study of Solar Flare Plasma", in A Crossroads for European Solar and Heliospheric Physics: Recent Achievements and Future Mission Possibilities, Proceedings of the ESA/IAC Conference, Puerto de la Cruz, Tenerife, Canary Islands, Spain, March 23-27 1998, (Eds.) Priest, E. R., Moreno-Insertis, F., Harris, R. A., ESA Special Publications, SP-417, p. 313, ESA Publications Division, Noordwijk. [ADS]. (Cited on page 8.)

Sylwester, J., Kuzin, S., Kotov, Y. D., Farnik, F. and Reale, F., 2008, "SphinX: A fast solar Photometer in X-rays", J. Astrophys. Astron., 29, 339-343. [DOI], [ADS]. (Cited on page 8.)

Sylwester, J., Kowalinski, M., Gburek, S. et al., 2012, "SphinX Measurements of the 2009 Solar Minimum X-Ray Emission", Astrophys. J., 751, 111. [DOI], [ADS], [arXiv:1203.6809 [astro-ph.SR]]. (Cited on page 19.)

Tajfirouze, E. and Safari, H., 2012, "Can a Nanoflare Model of Extreme-ultraviolet Irradiances Describe the Heating of the Solar Corona?", Astrophys. J., 744, 113. [DOI], [ADS], [arXiv:1112.4926 [astro-ph.SR]]. (Cited on pages 29, 46, and 52.)

Tanaka, Y., 1983, "Introduction to HINOTORI", Solar Phys., 86, 3-6. [DOI], [ADS]. (Cited on page 8.)

Taroyan, Y., 2009, "Alfvén Instability in Coronal Loops With Siphon Flows", Astrophys. J., 694, 69-75. [DOI], [ADS]. (Cited on page 48.)

Taroyan, Y., Erdélyi, R., Doyle, J. G. and Bradshaw, S. J., 2005, "Footpoint excitation of standing acoustic waves in coronal loops", Astron. Astrophys., 438, 713-720. [DOI], [ADS]. (Cited on page 48.)

Taroyan, Y., Bradshaw, S. J. and Doyle, J. G., 2006, "Nanoflare heating of coronal loops: hydrodynamic response and observational consequences", Astron. Astrophys., 446, 315-325. [DOI], [ADS]. (Cited on page 51.)

Telleschi, A., Güdel, M., Briggs, K., Audard, M., Ness, J.-U. and Skinner, S. L., 2005, "Coronal Evolution of the Sun in Time: High-Resolution X-Ray Spectroscopy of Solar Analogs with Different Ages", Astrophys. J., 622, 653-679. [DOI], [ADS], [arXiv:astro-ph/0503546]. (Cited on page 57.)

Teriaca, L., Doyle, J. G., Erdélyi, R. and Sarro, L. M., 1999, "New insight into transition region dynamics via SUMER observations and numerical modelling”, Astron. Astrophys., 352, L99-L102. [ADS]. (Cited on pages 29,30 , and 46. )

Teriaca, L., Madjarska, M. S. and Doyle, J. G., 2002, "Transition region explosive events: Do they have a coronal counterpart?", Astron. Astrophys., 392, 309-317. [DOI], [ADS]. (Cited on page 30.)

Teriaca, L., Banerjee, D., Falchi, A., Doyle, J. G. and Madjarska, M. S., 2004, "Transition region small-scale dynamics as seen by SUMER on SOHO", Astron. Astrophys., 427, 1065-1074. [DOI], [ADS]. (Cited on page 29.)

Teriaca, L., Warren, H. P. and Curdt, W., 2012, "Spectroscopic Observations of Fe XVIII in Solar Active Regions", Astrophys. J. Lett., 754, L40. [DOI], [ADS], [arXiv:1206.4228 [astro-ph.SR]]. (Cited on pages 21 and 45.$)$

Terzo, S. and Reale, F., 2010, "On the importance of background subtraction in the analysis of coronal loops observed with TRACE", Astron. Astrophys., 515, A7. [DOI], [ADS], [arXiv:1002.2121 [astro-ph.SR]]. (Cited on page 18.)

Terzo, S., Reale, F., Miceli, M., Klimchuk, J. A., Kano, R. and Tsuneta, S., 2011, "Widespread Nanoflare Variability Detected with Hinode/X-Ray Telescope in a Solar Active Region", Astrophys. J., 736, 111. [DOI], [ADS], [arXiv:1105.2506 [astro-ph.SR]]. (Cited on page 29.) 
Testa, P. and Reale, F., 2012, "Hinode/EIS Spectroscopic Validation of Very Hot Plasma Imaged with the Solar Dynamics Observatory in Non-flaring Active Region Cores", Astrophys. J. Lett., 750, L10. [DOI], [ADS], [arXiv:1204.0041 [astro-ph.SR]]. (Cited on pages 21 and 45.)

Testa, P., Peres, G., Reale, F. and Orlando, S., 2002, "Temperature and Density Structure of Hot and Cool Loops Derived from the Analysis of TRACE Data", Astrophys. J., 580, 1159-1171. [DOI], [ADS]. (Cited on pages 15 and 18.)

Testa, P., Peres, G. and Reale, F., 2005, "Emission Measure Distribution in Loops Impulsively Heated at the Footpoints", Astrophys. J., 622, 695-703. [DOI], [ADS], [arXiv:astro-ph/0412482]. (Cited on pages 51 and 52.)

Testa, P., De Pontieu, B., Martínez-Sykora, J., Hansteen, V. and Carlsson, M., 2012, "Investigating the Reliability of Coronal Emission Measure Distribution Diagnostics using Three-dimensional Radiative Magnetohydrodynamic Simulations", Astrophys. J., 758, 54. [DOI], [ADS], [arXiv:1208.4286 [astroph.SR]]. (Cited on page 19.)

Thomas, J. H., 1988, "Siphon flows in isolated magnetic flux tubes", Astrophys. J., 333, 407-419. [DOI], [ADS]. (Cited on page 46.)

Thomas, J. H. and Montesinos, B., 1990, "Siphon flows in isolated magnetic flux tubes. III. The equilibrium path of the flux-tube arch", Astrophys. J., 359, 550-559. [DOI], [ADS]. (Cited on page 46.)

Thomas, J. H. and Montesinos, B., 1991, "Siphon flows in isolated magnetic flux tubes. IV. Critical flows with standing tube shocks", Astrophys. J., 375, 404-413. [DOI], [ADS]. (Cited on page 46.)

Tian, H., McIntosh, S. W. and De Pontieu, B., 2011, "The Spectroscopic Signature of Quasi-periodic Upflows in Active Region Timeseries", Astrophys. J. Lett., 727, L37. [DOI], [ADS], [arXiv:1012.5112 [astro-ph.SR]]. (Cited on page 32.)

Title, A. M., 2010, "AIA on SDO", Bull. Am. Astron. Soc., 42, 308.03. [ADS]. (Cited on page 53.)

Tomczyk, S., McIntosh, S. W., Keil, S. L., Judge, P. G., Schad, T., Seeley, D. H. and Edmondson, J., 2007, "Alfvén Waves in the Solar Corona", Science, 317, 1192-1196. [DOI], [ADS]. (Cited on pages 33 and 54.)

Tousey, R., Bartoe, J.-D. F., Brueckner, G. E. and Purcell, J. D., 1977, "Extreme ultraviolet spectroheliograph ATM experiment S082A", Appl. Opt., 16, 870-878. [ADS]. (Cited on page 7.)

Tripathi, D., Mason, H. E., Young, P. R. and Del Zanna, G., 2008, "Density structure of an active region and associated moss using Hinode/EIS", Astron. Astrophys., 481, L53-L56. [DOI], [ADS], [arXiv:0802.3311]. (Cited on page 24.)

Tripathi, D., Mason, H. E., Dwivedi, B. N., Del Zanna, G. and Young, P. R., 2009, "Active Region Loops: Hinode/Extreme-Ultraviolet Imaging Spectrometer Observations", Astrophys. J., 694, 12561265. [DOI], [ADS], [arXiv:0901.0095]. (Cited on pages 15, 24, 26, 31, and 45.)

Tripathi, D., Mason, H. E. and Klimchuk, J. A., 2010, "Evidence of Impulsive Heating in Active Region Core Loops", Astrophys. J., 723, 713-718. [DOI], [ADS], [arXiv:1009.0663 [astro-ph.SR]]. (Cited on page 24.)

Tripathi, D., Klimchuk, J. A. and Mason, H. E., 2011, "Emission Measure Distribution and Heating of Two Active Region Cores", Astrophys. J., 740, 111. [DOI], [ADS], [arXiv:1107.4480 [astro-ph.SR]]. (Cited on page 24.)

Tripathi, D., Mason, H. E., Del Zanna, G. and Bradshaw, S., 2012a, "Observations of Plasma Upflow in a Warm Loop with Hinode/EIS", Astrophys. J. Lett., 754, L4. [DOI], [ADS], [arXiv:1206.3367 [astroph.SR]]. (Cited on page 31.) 
Tripathi, D., Mason, H. E. and Klimchuk, J. A., 2012b, "Active Region Moss: Doppler Shifts from Hinode/Extreme-ultraviolet Imaging Spectrometer Observations", Astrophys. J., 753, 37. [DOI], [ADS], [arXiv:1204.6550 [astro-ph.SR]]. (Cited on page 30.)

Tsuneta, S., Acton, L. W., Bruner, M. et al., 1991, "The soft X-ray telescope for the SOLAR-A mission", Solar Phys., 136, 37-67. [DOI], [ADS]. (Cited on page 8.)

Tsuneta, S., Hara, H., Shimizu, T., Acton, L. W., Strong, K. T., Hudson, H. S. and Ogawara, Y., 1992, "Observation of a solar flare at the limb with the YOHKOH Soft X-ray Telescope", Publ. Astron. Soc. Japan, 44, L63-L69. [ADS]. (Cited on page 20.)

Uchida, Y., 1970, "Diagnosis of Coronal Magnetic Structure by Flare-Associated Hydromagnetic Disturbances", Publ. Astron. Soc. Japan, 22, 341-364. [ADS]. (Cited on page 12.)

Ugarte-Urra, I. and Warren, H. P., 2011, "Temporal Variability of Active Region Outflows", Astrophys. J., 730, 37. [DOI], [ADS], [arXiv:1008.4730 [astro-ph.SR]]. (Cited on pages 29 and 32.)

Ugarte-Urra, I. and Warren, H. P., 2012, "Is Active Region Core Variability Age Dependent?", Astrophys. J., 761, 21. [DOI], [ADS]. (Cited on page 29.)

Ugarte-Urra, I., Warren, H. P. and Brooks, D. H., 2009, "Active Region Transition Region Loop Populations and Their Relationship to the Corona", Astrophys. J., 695, 642-651. [DOI], [ADS], [arXiv:0901.1075]. (Cited on page 22.)

Ulrich, R. K., 1996, "Observations of Magnetohydrodynamic Oscillations in the Solar Atmosphere with Properties of Alfvén Waves", Astrophys. J., 465, 436. [DOI], [ADS]. (Cited on page 32.)

Uritsky, V. M., Davila, J. M., Viall, N. M. and Ofman, L., 2013, "Measuring Temperature-dependent Propagating Disturbances in Coronal Fan Loops Using Multiple SDO/AIA Channels and the Surfing Transform Technique", Astrophys. J., 778, 26. [DOI], [ADS], [arXiv:1308.6195 [astro-ph.SR]]. (Cited on page 12.)

Uzdensky, D. A., 2007, "The Fast Collisionless Reconnection Condition and the Self-Organization of Solar Coronal Heating", Astrophys. J., 671, 2139-2153. [DOI], [ADS], [arXiv:0707.1316]. (Cited on page 56.)

Vaiana, G. S. and Rosner, R., 1978, "Recent advances in coronal physics", Annu. Rev. Astron. Astrophys., 16, 393-428. [DOI], [ADS]. (Cited on pages 6 and 7.)

Vaiana, G. S., Reidy, W. P., Zehnpfennig, T., Vanspeybroeck, L. and Giacconi, R., 1968, "X-ray Structures of the Sun during the Importance 1N Flare of 8 June 1968", Science, 161, 564-567. [DOI], [ADS]. (Cited on page 7.)

Vaiana, G. S., Krieger, A. S. and Timothy, A. F., 1973, "Identification and Analysis of Structures in the Corona from X-Ray Photography", Solar Phys., 32, 81-116. [DOI], [ADS]. (Cited on pages 7, 11, and 17.)

van Ballegooijen, A. A., 1986, "Cascade of magnetic energy as a mechanism of coronal heating", Astrophys. J., 311, 1001-1014. [DOI], [ADS]. (Cited on page 55.)

van Ballegooijen, A. A., Asgari-Targhi, M., Cranmer, S. R. and DeLuca, E. E., 2011, "Heating of the Solar Chromosphere and Corona by Alfvén Wave Turbulence", Astrophys. J., 736, 3. [DOI], [ADS], [arXiv:1105.0402 [astro-ph.SR]]. (Cited on page 55.)

van den Oord, G. H. J. and Mewe, R., 1989, "The X-ray flare and the quiescent emission from Algol as detected by EXOSAT", Astron. Astrophys., 213, 245-260. [ADS]. (Cited on page 42.)

van den Oord, G. H. J., Mewe, R. and Brinkman, A. C., 1988, "An EXOSAT observation of an X-ray flare and quiescent emission from the RS CVn binary $\sigma^{2}$ CrB", Astron. Astrophys., 205, 181-196. [ADS]. (Cited on page 42.) 
Vekstein, G., 2009, "Probing nanoflares with observed fluctuations of the coronal EUV emission", Astron. Astrophys., 499, L5-L8. [DOI], [ADS]. (Cited on pages 15 and 51.)

Vekstein, G. and Katsukawa, Y., 2000, "Scaling Laws for a Nanoflare-Heated Solar Corona", Astrophys. J., 541, 1096-1103. [DOI], [ADS]. (Cited on page 52.)

Vekstein, G. E. and Jain, R., 2003, "Signatures of a nanoflare heated solar corona", Plasma Phys. Control. Fusion, 45, 535-545. [DOI], [ADS]. (Cited on page 52.)

Velli, M., Hood, A. W. and Einaudi, G., 1990, "Ideal kink instabilities in line-tied coronal loops: Growth rates and geometrical properties", Astrophys. J., 350, 428-436. [DOI], [ADS]. (Cited on page 49.)

Verdini, A., Grappin, R. and Velli, M., 2012, "Coronal heating in coupled photosphere-chromospherecoronal systems: turbulence and leakage", Astron. Astrophys., 538, A70. [DOI], [ADS], [arXiv:1111.5359 [astro-ph.SR]]. (Cited on page 56.)

Vernazza, J. E., Avrett, E. H. and Loeser, R., 1981, "Structure of the Solar Chromosphere. III. Models of the EUV Brightness Components of the Quiet Sun", Astrophys. J. Suppl. Ser., 45, 635-725. [DOI], [ADS]. (Cited on page 35.)

Verwichte, E., Nakariakov, V. M. and Longbottom, A. W., 1999, "On the evolution of a nonlinear Alfvén pulse", J. Plasma Phys., 62, 219-232. [DOI], [ADS]. (Cited on page 54.)

Vesecky, J. F., Antiochos, S. K. and Underwood, J. H., 1979, "Numerical modeling of quasi-static coronal loops. I. Uniform energy input", Astrophys. J., 233, 987-997. [DOI], [ADS]. (Cited on pages 34 and 37.)

Viall, N. M. and Klimchuk, J. A., 2011, "Patterns of Nanoflare Storm Heating Exhibited by an Active Region Observed with Solar Dynamics Observatory/Atmospheric Imaging Assembly", Astrophys. J., 738, 24. [DOI], [ADS]. (Cited on page 29.)

Viall, N. M. and Klimchuk, J. A., 2012, "Evidence for Widespread Cooling in an Active Region Observed with the SDO Atmospheric Imaging Assembly", Astrophys. J., 753, 35. [DOI], [ADS], [arXiv:1202.4001 [astro-ph.SR]]. (Cited on page 29.)

Wang, J., Shibata, K., Nitta, N., Slater, G. L., Savy, S. K. and Ogawara, Y., 1997, "Shrinkage of Coronal X-Ray Loops", Astrophys. J. Lett., 478, L41. [DOI], [ADS]. (Cited on page 27.)

Wang, T., Ofman, L., Davila, J. M. and Su, Y., 2012, "Growing Transverse Oscillations of a Multistranded Loop Observed by SDO/AIA", Astrophys. J. Lett., 751, L27. [DOI], [ADS], [arXiv:1204.1376 [astroph.SR]]. (Cited on pages 33 and 45.)

Wang, T., Ofman, L. and Davila, J. M., 2013, "Three-dimensional Magnetohydrodynamic Modeling of Propagating Disturbances in Fan-like Coronal Loops", Astrophys. J. Lett., 775, L23. [DOI], [ADS], [arXiv:1308.0282 [astro-ph.SR]]. (Cited on pages 12 and 32.)

Wang, T. J., Ofman, L. and Davila, J. M., 2009, "Propagating Slow Magnetoacoustic Waves in Coronal Loops Observed by Hinode/EIS", Astrophys. J., 696, 1448-1460. [DOI], [ADS], [arXiv:0902.4480 [astroph.SR]]. (Cited on page 55.)

Warren, H. P., 2006, "Multithread Hydrodynamic Modeling of a Solar Flare", Astrophys. J., 637, 522-530. [DOI], [ADS], [arXiv:astro-ph/0507328]. (Cited on page 40.)

Warren, H. P. and Winebarger, A. R., 2006, "Hydrostatic Modeling of the Integrated Soft X-Ray and Extreme Ultraviolet Emission in Solar Active Regions", Astrophys. J., 645, 711-719. [DOI], [ADS], [arXiv:astro-ph/0602052]. (Cited on pages 36 and 45.)

Warren, H. P. and Winebarger, A. R., 2007, "Static and Dynamic Modeling of a Solar Active Region", Astrophys. J., 666, 1245-1255. [DOI], [ADS], [arXiv:astro-ph/0609023]. (Cited on page 51.) 
Warren, H. P., Winebarger, A. R. and Hamilton, P. S., 2002, "Hydrodynamic Modeling of Active Region Loops", Astrophys. J. Lett., 579, L41-L44. [DOI], [ADS]. (Cited on pages 25, 36, 44, 45, and 51.)

Warren, H. P., Winebarger, A. R. and Mariska, J. T., 2003, "Evolving Active Region Loops Observed with the Transition Region and Coronal explorer. II. Time-dependent Hydrodynamic Simulations", Astrophys. J., 593, 1174-1186. [DOI], [ADS]. (Cited on pages 25, 44, 45, and 51.)

Warren, H. P., Ugarte-Urra, I., Doschek, G. A., Brooks, D. H. and Williams, D. R., 2008a, "Observations of Active Region Loops with the EUV Imaging Spectrometer on Hinode", Astrophys. J. Lett., 686, L131-L134. [DOI], [ADS], [arXiv:0808.3227]. (Cited on pages 25 and 26.)

Warren, H. P., Winebarger, A. R., Mariska, J. T., Doschek, G. A. and Hara, H., 2008b, "Observation and Modeling of Coronal 'Moss' With the EUV Imaging Spectrometer on Hinode", Astrophys. J., 677, 1395-1400. [DOI], [ADS], [arXiv:0709.0396]. (Cited on page 25.)

Warren, H. P., Kim, D. M., DeGiorgi, A. M. and Ugarte-Urra, I., 2010a, "Modeling Evolving Coronal Loops with Observations from Stereo, Hinode, and Trace", Astrophys. J., 713, 1095-1107. [DOI], [ADS], [arXiv:0904.3920 [astro-ph.SR]]. (Cited on pages 26 and 52.)

Warren, H. P., Winebarger, A. R. and Brooks, D. H., 2010b, "Evidence for Steady Heating: Observations of an Active Region Core with Hinode and TRACE", Astrophys. J., 711, 228-238. [DOI], [ADS], [arXiv:0910.0458 [astro-ph.SR]]. (Cited on pages 27, 28, and 52.)

Warren, H. P., Brooks, D. H. and Winebarger, A. R., 2011, "Constraints on the Heating of Hightemperature Active Region Loops: Observations from Hinode and the Solar Dynamics Observatory", Astrophys. J., 734, 90. [DOI], [ADS], [arXiv:1009.5976 [astro-ph.SR]]. (Cited on pages 9, 24, and 28.)

Warren, H. P., Winebarger, A. R. and Brooks, D. H., 2012, "A Systematic Survey of High-temperature Emission in Solar Active Regions", Astrophys. J., 759, 141. [DOI], [ADS], [arXiv:1204.3220 [astroph.SR]]. (Cited on page 21.)

Watanabe, T., Hara, H., Yamamoto, N. et al., 2009, "Fe XIII Density Diagnostics in the EIS Observing Wavelengths", Astrophys. J., 692, 1294-1304. [DOI], [ADS]. (Cited on page 20.)

Weber, M. A., Schmelz, J. T., DeLuca, E. E. and Roames, J. K., 2005, "Isothermal Bias of the 'Filter Ratio' Method for Observations of Multithermal Plasma”, Astrophys. J. Lett., 635, L101-L104. [DOI], [ADS]. (Cited on pages 17, 18, and 25.)

West, M. J., Bradshaw, S. J. and Cargill, P. J., 2008, "On the Lifetime of Hot Coronal Plasmas Arising from Nanoflares", Solar Phys., 252, 89-100. [DOI], [ADS]. (Cited on page 36.)

White, S. M., Kundu, M. R. and Gopalswamy, N., 1991, "Strong magnetic fields and inhomogeneity in the solar corona", Astrophys. J. Lett., 366, L43-L46. [DOI], [ADS]. (Cited on page 12.)

Wiegelmann, T., Inhester, B., Kliem, B., Valori, G. and Neukirch, T., 2006, "Testing non-linear force-free coronal magnetic field extrapolations with the Titov-Démoulin equilibrium", Astron. Astrophys., 453, 737-741. [DOI], [ADS], [astro-ph/0612650]. (Cited on page 7.)

Wilhelm, K., Curdt, W., Marsch, E. et al., 1995, "SUMER - Solar Ultraviolet Measurements of Emitted Radiation", Solar Phys., 162, 189-231. [DOI], [ADS]. (Cited on page 8.)

Wilmot-Smith, A. L., Pontin, D. I. and Hornig, G., 2010, "Dynamics of braided coronal loops. I. Onset of magnetic reconnection", Astron. Astrophys., 516, A5. [DOI], [ADS], [arXiv:1001.1717 [astro-ph.SR]]. (Cited on page 50.)

Wilmot-Smith, A. L., Pontin, D. I., Yeates, A. R. and Hornig, G., 2011, "Heating of braided coronal loops", Astron. Astrophys., 536, A67. [DOI], [ADS], [arXiv:1111.1100 [astro-ph.SR]]. (Cited on page 50.)

Winebarger, A., Tripathi, D., Mason, H. E. and Del Zanna, G., 2013, "Doppler Shifts in Active Region Moss Using SOHO/SUMER", Astrophys. J., 767, 107. [DOI], [ADS]. (Cited on page 29.) 
Winebarger, A. R. and Warren, H. P., 2004, "Can TRACE Extreme-Ultraviolet Observations of Cooling Coronal Loops Be Used to Determine the Heating Parameters?", Astrophys. J. Lett., 610, L129-L132. [DOI], [ADS]. (Cited on page 51.)

Winebarger, A. R. and Warren, H. P., 2005, "Cooling Active Region Loops Observed with SXT and TRACE", Astrophys. J., 626, 543-550. [DOI], [ADS], [arXiv:astro-ph/0502270]. (Cited on pages 15 and 38.)

Winebarger, A. R., Emslie, A. G., Mariska, J. T. and Warren, H. P., 1999, "Analyzing the Energetics of Explosive Events Observed by SUMER on SOHO", Astrophys. J., 526, 471-477. [DOI], [ADS]. (Cited on page 30.)

Winebarger, A. R., DeLuca, E. E. and Golub, L., 2001, "Apparent Flows above an Active Region Observed with the Transition Region and Coronal Explorer", Astrophys. J. Lett., 553, L81-L84. [DOI], [ADS]. (Cited on pages 31 and 45.)

Winebarger, A. R., Emslie, A. G., Mariska, J. T. and Warren, H. P., 2002a, "Energetics of Explosive Events Observed with SUMER", Astrophys. J., 565, 1298-1311. [DOI], [ADS]. (Cited on page 30.)

Winebarger, A. R., Updike, A. C. and Reeves, K. K., 2002b, "Correlating Transition Region Explosive Events with Extreme-Ultraviolet Brightenings", Astrophys. J. Lett., 570, L105-L108. [DOI], [ADS]. (Cited on page 30.)

Winebarger, A. R., Warren, H., van Ballegooijen, A. A., DeLuca, E. E. and Golub, L., 2002c, "Steady Flows Detected in Extreme-Ultraviolet Loops", Astrophys. J. Lett., 567, L89-L92. [DOI], [ADS]. (Cited on pages 31,45 , and 48 .)

Winebarger, A. R., Warren, H. P. and Mariska, J. T., 2003a, "Transition Region and Coronal Explorer and Soft X-Ray Telescope Active Region Loop Observations: Comparisons with Static Solutions of the Hydrodynamic Equations", Astrophys. J., 587, 439-449. [DOI], [ADS]. (Cited on pages 25, 38, 44, and 45.)

Winebarger, A. R., Warren, H. P. and Seaton, D. B., 2003b, "Evolving Active Region Loops Observed with the Transition Region and Coronal Explorer. I. Observations", Astrophys. J., 593, 1164-1173. [DOI], [ADS]. (Cited on pages 44, 45, and 52.)

Winebarger, A. R., Warren, H. P. and Falconer, D. A., 2008, "Modeling X-Ray Loops and EUV 'Moss' in an Active Region Core", Astrophys. J., 676, 672-679. [DOI], [ADS], [arXiv:0712.0756]. (Cited on page 38.)

Winebarger, A. R., Schmelz, J. T., Warren, H. P., Saar, S. H. and Kashyap, V. L., 2011, "Using a Differential Emission Measure and Density Measurements in an Active Region Core to Test a Steady Heating Model", Astrophys. J., 740, 2. [DOI], [ADS], [arXiv:1106.5057 [astro-ph.SR]]. (Cited on page 53.)

Winebarger, A. R., Warren, H. P., Schmelz, J. T., Cirtain, J., Mulu-Moore, F., Golub, L. and Kobayashi, K., 2012, "Defining the "Blind Spot" of Hinode EIS and XRT Temperature Measurements", Astrophys. J. Lett., 746, L17. [DOI], [ADS]. (Cited on pages 19 and 21.)

Woods, T. N., Eparvier, F. G., Hock, R. et al., 2012, "Extreme Ultraviolet Variability Experiment (EVE) on the Solar Dynamics Observatory (SDO): Overview of Science Objectives, Instrument Design, Data Products, and Model Developments", Solar Phys., 275, 115-143. [DOI], [ADS]. (Cited on page 8.)

Wragg, M. A. and Priest, E. R., 1981, "The temperature-density structure of coronal loops in hydrostatic equilibrium", Solar Phys., 70, 293-313. [DOI], [ADS]. (Cited on page 38.)

Yokoyama, T. and Shibata, K., 2001, "Magnetohydrodynamic Simulation of a Solar Flare with Chromospheric Evaporation Effect Based on the Magnetic Reconnection Model", Astrophys. J., 549, 1160-1174. [DOI], [ADS]. (Cited on page 37.) 
Yoshida, T. and Tsuneta, S., 1996, "Temperature Structure of Solar Active Regions", Astrophys. J., 459, 342. [DOI], [ADS]. (Cited on page 20.)

Yoshida, T., Tsuneta, S., Golub, L., Strong, K. and Ogawara, Y., 1995, "Temperature Structure of the Solar Corona: Comparison of the NIXT and YOHKOH X-Ray Images", Publ. Astron. Soc. Japan, 47, L15-L19. [ADS]. (Cited on page 21.)

Young, P. R., Watanabe, T., Hara, H. and Mariska, J. T., 2009, "High-precision density measurements in the solar corona. I. Analysis methods and results for Fe XII and Fe XIII", Astron. Astrophys., 495, 587-606. [DOI], [ADS], [arXiv:0805.0958]. (Cited on page 20.)

Zacharias, P., Peter, H. and Bingert, S., 2011, "Ejection of cool plasma into the hot corona", Astron. Astrophys., 532, A112. [DOI], [ADS], [arXiv:1106.5972 [astro-ph.SR]]. (Cited on page 48.)

Zhang, Y. Z., Shibata, K., Wang, J. X., Mao, X. J., Matsumoto, T., Liu, Y. and Su, J. T., 2012, "Revision of Solar Spicule Classification", Astrophys. J., 750, 16. [DOI], [ADS], [arXiv:1202.4518 [astro-ph.SR]]. (Cited on page 32.)

Zhitnik, I. A., Bugaenko, O. I., Ignat'ev, A. P. et al., 2003, "Dynamic 10 MK plasma structures observed in monochromatic full-Sun images by the SPIRIT spectroheliograph on the CORONAS-F mission", Mon. Not. R. Astron. Soc., 338, 67-71. [DOI], [ADS]. (Cited on page 8.)

Zirker, J. B., 1993, "Coronal heating”, Solar Phys., 148, 43-60. [DOI], [ADS]. (Cited on page 6.) 